M. Aschbacher and G. M. Seitz

Nagoya Math. J.

Vol. 63 (1976), 1-91

\title{
INVOLUTIONS IN GHEVALLEY GROUPS OVER FIELDS OF EVEN ORDER
}

\section{MICHAEL ASCHBACHER* AND GARY M. SEITZ**}

Let $G=G(q)$ be a Chevalley group defined over a field $F_{q}$ of characteristic 2. In this paper we determine the conjugacy classes of involutions in Aut $(G)$ and the centralizers of these involutions. This study was begun in the context of a different problem. Namely, we wanted to find those groups $H$ containing a standard component $A$ satisfying $A / Z(A) \cong G$ and $m_{2}\left(C_{H}(A)\right)>1$. Such groups $H$ are determined in [3], where the results of this paper are crucial. In dealing with groups $H$ as above a very important consideration is the tightly embedded subgroups in $\operatorname{Aut}(G)$. Consequently we study such subgroups in this paper.

The classical groups and the exceptional Chevalley groups are treated separately. Finding the involutions in the classical groups is accomplished using the underlying vector space and regarding the group as a group of matrices. The results give an explicit matrix representation for the involutions in $G$ and their centralizers. For the exceptional groups the $(B, N)$-structure is used. Representatives for the classes of involutions are given as explicit products of elements of root groups. The centralizers are determined completely; one could obtain precise generators and relations if needed. One useful piece of information is the complete list of parabolic subgroups containing a given centralizer of an involution.

The analysis of outer automorphisms is carried out in the context of the $(B, N)$-structure, with the exception of the orthogonal groups where certain information is already available from the linear algebra.

We remark that for $t$ an involution in $G, C_{G}(t)$ is known to be 2constrained with $O_{2^{\prime}}\left(C_{G}(t)\right)=1$. It turns out that $O_{2}\left(C_{G}(t)\right)$ is always of class at most 3 ; for the classical groups the class is at most 2 .

Received June 3, 1975.

* Partial support supplied by the Alfred P. Sloan Foundation and NSF GD-35678.

** Partial support supplied by NSF GRANT 37982XI. 
The paper is organized as follows. $\S 2$ contains preliminary results and $\S 3$ contains a discussion of groups with $(B, N)$-pairs, giving commutator relations and root systems for the exceptional Chevalley groups. Sections 4-8 are concerned with the involutions in the classical groups. Included is a description of the underlying geometric configuration. Sections 9-11 give technical information needed in the analysis of the structure of the centralizers and for finding the tightly embedded subgroups. The involutions in the rank 2 Chevalley groups have been previously determined, so in $\S 12$ we find the involutions in the remaining exceptional Chevalley groups. Then Sections 13-18 contain the precise information on the centralizers of these involutions. $\S 19$ is concerned with the outer automorphisms. In $\S 20$ we prove that certain types of tightly embedded subgroups in Aut $(G)$ have all their involutions in $G$. Finally in $\S 21$ and $\S 22$ we return to the classical groups with a discussion of exceptional Schur multipliers and further analysis of tightly embedded subgroups.

\section{Section 2. Preliminaries.}

In this section we record some preliminary lemmas to be used in later sections, especially Section 20.

(2.1) Let $G=P Q A$ be the semi-direct product of the product of the $p$ group $P \unlhd G$ and the group $Q A$. Assume that $A$ is a cyclic p-group acting fixed-point-freely on $Q$, and that $C_{P}(Q)=1$. Then all complements to $P Q$ in $G$ are conjugate.

Proof. This is contained in Theorem 3.3 of [20].

(2.2) Let $t$ be an involution in a group $D$. Then $L\left(C_{D}(t)\right) \leq L(D)$ and if $F^{*}(D)$ is a 2-group then $O\left(C_{D}(t)\right)=1$.

Proof. See 2.6 and 2.7 of [2].

(2.3) Let $G$ be a finite Chevalley group (normal or twisted) defined over a field of characteristic $p$. Then p-local subgroups of $G$ are contained in proper parabolic subgroups.

Proof. Borel-Tits, 3.12 of [4].

(2.4) Let $G$ be a finite Chevalley group (normal or twisted) defined over 
a field of characteristic $p$. Then for any p-element $x \neq 1, F^{*}\left(C_{G}(x)\right)$ is a p-group.

Proof. This follows from 2.3 and the fact that for proper parabolic subgroups, $P$ of $G, F^{*}(P)$ is a $p$-group.

(2.5) If $X$ is a 2-group of rank $>1$ acting on a group $Y$ of odd order, then $Y=\left\langle C_{Y}(x): x \in X^{\#}\right\rangle=\Gamma_{1, X}(Y)$.

Proof. See Gorenstein [12].

\section{Section 3. The $(B, N)$-structure of the exceptional groups.}

Throughout most of this paper we will be concerned with determining the involutions, and their centralizers, in the Chevalley groups $G$ defined over fields of characteristic 2. In the case of the classical groups the representation of $G$ on its natural module is used to obtain this information. However in the case of the exceptional groups we must utilize the $(B, N)$-structure of $G$ and work entirely within local subgroups. Moreover in the case of the classical groups our results are stated in terms of the natural modules while in the case of the exceptional groups our results are given explicitly in terms of the $(B, N)$ structure.

Therefore in this section we record notation and basic facts concerning the $(B, N)$-structure of the exceptional groups.

We write $G=G(q)$ to mean that $G$ is defined over a field $\boldsymbol{F}_{q}$ of characteristic 2. Fix a Tit's system for $G$. So let $U$ be a Sylow 2subgroup of $G$ with $B=N_{G}(U)$ and $U=O_{2}(B)$. Then $B=U H$ where $H$ is an abelian 2 -group normal in $N$. Write $W=N / H$, the Weyl group, $W=\left\langle s_{1}, \cdots, s_{n}\right\rangle$, where the $s_{i}$ are the fundamental reflections. Associated with $W$ is a root system $\Delta$ in $\boldsymbol{R}^{n}$ with positive roots $\Delta^{+}$and fundamental system $\left\{\alpha_{1}, \cdots, \alpha_{n}\right\}$. We label the Dynkin diagram of $G$ and the roots in $\Delta$ as in Bourbaki [5].

For each root $r \in \Delta$ there corresponds a subgroup (called a "root group") $U_{r} \leq G$ such that $G=\left\langle U_{r}: r \in \Delta\right\rangle$ and $U=\prod_{r \in \Delta^{+}} U_{r}$ in some fixed ordering of $\Delta^{+}$. Then $H$ normalizes each $U_{r}$ and the permutation representation of $W$ on $\Delta$ and on $\left\{U_{r}: r \in \Delta\right\}$ are equivalent under the correspondence $r \leftrightarrow U_{r}$. For $i=1, \cdots, n$ let $s_{i}=U_{-\alpha_{i}}(1) U_{\alpha_{i}}(1) U_{-\alpha_{i}}(1)$. Here we are labeling the elements of $U_{r}$ by using the base field (or other 
fields for the twisted groups) and we are identifying $s_{i}$ as both a coset of $H$ in $W$ and as an element in $G$. This will not cause difficulty and it is true that $U_{-\alpha_{i}}(1) U_{\alpha_{i}}(1) U_{-\alpha_{i}}(1) H=s_{i} \in W$.

For $i=1, \cdots, n$ let $\mathscr{L}^{i}$ be the set of all roots in $\Delta$ with positive coefficient of $\alpha_{i}$. Then $\mathscr{L}^{i} \subseteq \Delta^{+}$. If $i=1, \cdots, n$ and $m \geq 0$, let $\mathscr{L}_{m}^{i}$ denote the set of long roots with coefficient of $\alpha_{i}$ equal to $m$ (respectively $\mathscr{S}_{m}^{i}$ for the short roots). We write $Q_{i}=\left\langle U_{r}: r \in \mathscr{L}^{i}\right\rangle$ and set $Q_{j}^{i}=\left\{U_{s}: s \in \mathscr{L}_{j}^{i}\right\}$.

For $S \subset\{1, \cdots, n\}$ we let $P_{S}=\left\langle B, s_{i}: i \in S\right\rangle$. The conjugates of the subgroups $P_{S}$ are called the parabolic subgroups of $G$, and some information concerning the structure of $P_{S}$ can be obtained simply by looking at the Dynkin diagram. Namely $P_{S}=Q_{S} L_{S} H$, where $Q_{S}=O_{2}\left(P_{S}\right)$, $L_{S}=\left\langle U_{ \pm \alpha_{i}}: i \notin S\right\rangle=\left\langle U_{\alpha_{i}}, s_{i}: i \notin S\right\rangle$ and the structure of $L_{S}$ as a Chevalley group can be read off from the Dynkin diagram. The group $Q_{S}$ is the product of the groups $U_{r}$ for $r \in \bigcup_{i \in \mathscr{S}} \mathscr{L}^{i}$.

We will need the following cases of the Chevalley commutator relations.

(3.1) Let $\alpha, \beta \in \Delta$.

i) If $\alpha+\beta \notin \Delta$, then $\left[U_{\alpha}, U_{\beta}\right]=1$.

ii) If $\alpha, \beta, \alpha+\beta$ are all in $\Delta$ and of the same length, then $\left[U_{\alpha}(s), U_{\beta}(t)\right]=U_{\alpha+\beta}(s t)$.

iii) If $G=F_{4}(q), \alpha$ and $\beta$ short roots, and $\alpha+\beta$ a long root in $\Delta$, then $\left[U_{\alpha}, U_{\beta}\right]=1$.

iv) If $G={ }^{2} E_{6}(q), \alpha$ and $\beta$ short roots, and $\alpha+\beta$ a long root in $\Delta$, then $\left[U_{\alpha}(s), U_{\beta}(t)\right]=U_{\alpha+\beta}\left(s^{q} t+s t^{q}\right)$, for $s, t \in \boldsymbol{F}_{q^{2}}$.

v) If $G=F_{4}(q), q>2, \alpha$ is a long root, $\beta$ a short root such that $\alpha+\beta \in \Delta$, then $\left[U_{\alpha}, U_{\beta}\right]=U_{\alpha+\beta} U_{\alpha+2 \beta}$.

vi) If $G={ }^{2} E_{6}(q), \alpha$ a long root, $\beta$ a short root and $\alpha+\beta \in \Delta$, then $\left[U_{\alpha}(c), U_{\beta}(d)\right]=U_{\alpha+\beta}(c d) U_{\alpha+2 \beta}\left(c d d^{q}\right)$, for $c \in \boldsymbol{F}_{q}$ and $d \in \boldsymbol{F}_{q^{2}}$.

Basic facts involving parabolic subgroups and the Bruhat decomposition of $G$ will be used repeatedly. Also we will need a list of some of the roots in $\Delta^{+}$for $\Delta$ of type $F_{4}, E_{6}, E_{7}$, and $E_{8}$. These are given in the following tables and are based on the following labeling of the Dynkin diagram 
$F_{4}$

$E_{6}$

$E_{7}$

$E_{8}$

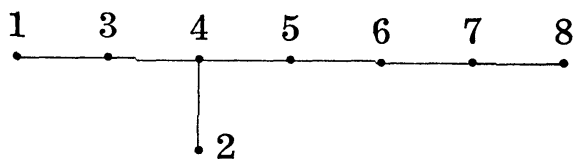

TABLE 1

Roots in $\Delta^{+}$for $\Delta$ of type $F_{4}$

$\mathscr{L}_{1}^{1}$

1. 1000

9. 1110

2. 1100

3. 1120

10. 1111

11. 1121

12. 1221

4. 1220

13. 1231

5. 1122

6. 1222

7. 1242

8. 1342

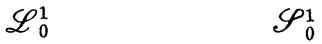

15. $0100 \quad 18.0010$

16. 0120 19. 0110

$\begin{array}{llll}17 . & 0122 & 20 . & 0111\end{array}$

21. 0121

22. 0001

23. 0011
$\mathscr{L}_{2}^{1}$

24. 2342

$$
\text { Let } \begin{aligned}
r & =r_{24} \\
s & =r_{14} \\
\alpha & =r_{12} \\
\beta & =r_{7}
\end{aligned}
$$

TABLE 2

Roots in $\Delta^{+}$for $\Delta$ of type $E_{6}$
$\mathscr{L}_{1}^{1}$
$\mathscr{L}_{1}^{2}-\mathscr{L}_{1}^{1}$
$\Delta^{+}-\mathscr{L}_{1}^{1}-\mathscr{L}_{1}^{2}$
1. 10000
17. 00000
0
1
27. 01000 
2. 11000

0

3. 11100

0

4. 11100

1

5. 11110

0

6. 11110

1

7. 11111

1

8. 11111

0

9. 11210

1

10. 11211

1

11. 12210

1

12. 12211

1

13. 11221

1

14. 12221

1

15. 12321

1

16. 12321
18. 00100

1

19. 01100

1

20. 00110

1

21. 00111

1

22. 01110

1

23. 01111

1

24. 01210

1

25. 01211

1

26. 01221

1
28. 01100

29. 01110

30. 01111

0

31. 00100

0

32. 00110

33. 00111

0

34. 00010

0

35. 00011

36. 00001 0

TABLE 3

Roots in $\Delta^{+}$having non-zero coefficient of $\alpha_{2}$ or $\alpha_{7}$, for $\Delta$ of type $E_{7}$.
$\mathscr{L}_{1}^{7} \cap \mathscr{L}_{0}^{2}$
$\mathscr{L}_{1}^{7} \cap \mathscr{L}_{1}^{2}$
$\mathscr{L}_{1}^{7} \cap \mathscr{L}_{2}^{2}$
1. 000001
7. 001111
0
1
22. 123211
2. 000011
8. 011111
2
0
1
23. 123221
3. 000111
9. 111111
2
0
. 1111
4. 001111
10. 012111
1
24. 123321
2
25. 124321
2 

5. 011111
0
6. 111111
0

11. 112111

1

12. 012211

1

13. 112211

1

$r=r_{27}$

$\alpha=r_{21}$

$\beta=r_{23}$

$\gamma=r_{48}$

$\delta=r_{18}$

$\varepsilon=r_{19}$

$\varphi=r_{13}$

$\psi=r_{14}$

$\theta=r_{15}$

$\mathscr{L}_{0}^{7} \cap \mathscr{L}^{2} \cap \mathscr{L}_{1}^{1}$

38. 111000

1

39. 111100

1

40. 111110

1
14. 122111

1

15. 012221

1

16. 122211

1

17. 112221

1

18. 122221

1

19. 123211

1

20. 123221

1

21. 123321

1
26. 134321

2

27. 234321

2

$\mathscr{L}_{0}^{7} \cap \mathscr{L}_{1}^{2} \cap \mathscr{L}_{0}^{1}$

28. 000000

1

29. 001000 1

30. 011000

1

31. 001100

1

32. 001110 1

33. 011100

1

34. 011110

35. 012100

1

36. 012110

1

$\begin{array}{cc}37 . & 012210 \\ 1\end{array}$ 


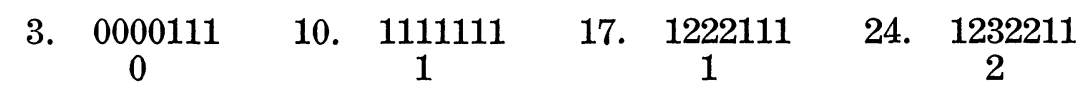

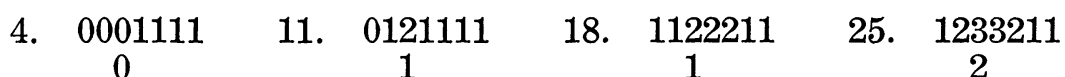

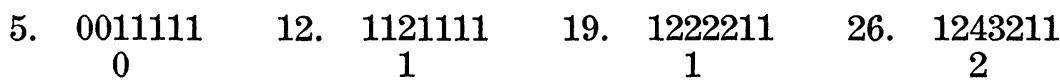

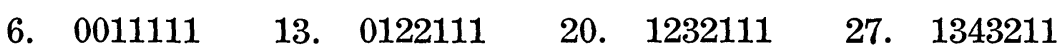
1
13. 1
12

7. 0111111 14. 1122111 $\begin{array}{ccc}0 & 1 \\ 0111111 & 15 . & 1221111\end{array}$

8. 0111111

1

1

21. 1232211

1

28. 2343211

22. 1233211

$\mathscr{L}^{8} \cap\left(\mathscr{L}_{2}^{7} \cup \mathscr{L}_{3}^{7}\right)$

29. $1343221 \quad 37.1233221$

2

2

44. 2343321

2

51. 2454321

30. 1243321

2

38. 1233321

1

45. 1344321

2

52. 2454321

31. $0122221 \quad 39 . \quad 1243221$

1

2

46. 1354321

2

53. 2464321

32. 0122221

1

40. 1233321

2

47. 2344321

2

33. 1222221

1
34. 1232221

41. 2343221

2

48. 1354321

3

1

42. 1343321

2

3

3

49. 2354321

2

54. 2465321

3

55. 2465421

35. 1232221

43. 1244321

2

50. 2354321

3

3

56. 2465431

3

2

3

57. 2465432

36. 1233221

1

$\begin{array}{lllll}r=r_{57} & \beta=r_{50} & \delta=r_{42} & \varphi=2343210 & \theta \\ \alpha=r_{51} & \gamma=r_{41} & \varepsilon=r_{43} & \psi=r_{27} & \omega=r_{39}\end{array}$

\section{Section 4. The unimodular group.}

Let $V$ be an $n$-dimensional vector space over $G F(q)$, where $n \geq 3$, and $q$ is even. $S L(V)$ is the group of all linear transformations of $V$ of determinant 1 . Given an ordered basis $\left\{x_{i}\right\}$ of $V$ and $g \in S L(V)$, associate with $g$ the $n \times n$ matrix $\left(g_{i j}\right)$ where

$$
x_{i}^{g}=\sum_{j} g_{i j} x_{j} .
$$

In considering such matrices we follow the notation of M. Suzuki in [22]. 
Given an involution $a$ in $S L(V)$, define the rank of $a$ to be the dimension of the commutator space $[V, a]$ of $a$. The rank of $a$ is also the number of Jordan blocks of $\left(a_{i j}\right)$ of size 2 with respect to a basis of $V$ in which $a$ is in Jordan form. Hence

(4.1) Let $a$ and $b$ be involutions in $S L(V)$. Then $a$ and $b$ are conjugate in $S L(V)$ if and only if they have the same rank.

Fix an ordered basis for $V$ and associate each element of $S L(V)$ with its corresponding matrix. Following Suzuki, given an integer $\ell$ in the range $1 \leq \ell \leq n / 2$, define the involution $j_{\ell}$ of $S L(V)$ by

$$
j_{\ell}=\left[\begin{array}{lll}
I_{\ell} & & \\
& I_{n-2 \ell} & \\
I_{\ell} & & I_{\ell}
\end{array}\right]
$$

Here $I_{m}$ is the $m \times m$ identity matrix and $I_{0}$ is taken to be void.

$j_{\ell}$ has rank $\ell$ and is referred to as the Suzuki form of its class. We conclude

(4.2) The involutions $j_{\ell}, 1 \leq \ell \leq n / 2$, are a set of representatives for the conjugacy classes of involutions of $S L(V)$.

(4.3) The centralizer $C_{\ell}$ in $S L(V)$ of the involution $j_{\ell}$ consists of those matrices $g$ of the form

$$
g=\left[\begin{array}{lll}
X(g) & & \\
P(g) & Y(g) & \\
Q(g) & R(g) & X(g)
\end{array}\right] \text { such that }(\operatorname{det}(X))^{2} \operatorname{det}(Y)=1,
$$

where $X(g)$ and $Q(g)$ are of size $\ell \times \ell, Y(g)$ has size $(n-2 \ell) \times(n-2 \ell)$, $P(g)$ has size $(n-2 \ell) \times \ell$, and $R(g)$ has size $\ell \times(n-2 \ell)$. Further the map $g \rightarrow(X(g), Y(g))$ is a homomorphism of $C_{\ell}$ into $G L_{\ell}(q) \times G L_{n-2 \ell}(q)$ with the image containing $S L_{\ell}(q) \times S L_{n-2 \ell}(q)$, and covering both factors if $n \neq 2 \ell$. The kernel $T_{\ell}$ is equal to $\mathrm{O}_{2}\left(C_{\ell}\right)$.

Proof. See Suzuki [22], pages 1048 and 1049.

The remaining three lemmas in this section follow by direct computation.

(4.4) For $g, h \in C_{\ell}$ 
$g h=\left[\begin{array}{lll}X(g) X(h) & \\ P(g) X(h)+Y(g) P(h) & Y(g) Y(h) & \\ Q(g) X(h)+R(g) P(h)+X(g) Q(h) & R(g) Y(h)+X(g) R(h) & X(g) X(h)\end{array}\right]$.

(4.5) Let $g, h \in C_{\ell}$ with

$$
h=\left[\begin{array}{lll}
D & & \\
A & W & \\
B & C & D
\end{array}\right] \quad g=\left[\begin{array}{lll}
X & & \\
P & Y & \\
Q & R & X
\end{array}\right]
$$

Then

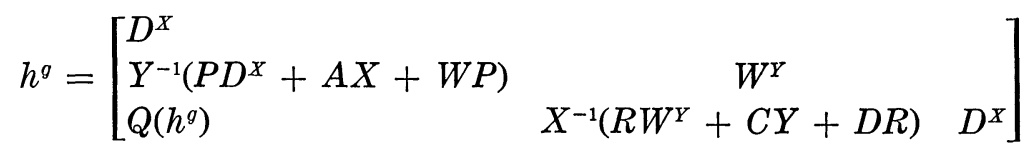

where

$$
Q\left(h^{g}\right)=X^{-1}\left[R Y^{-1}\left(P D^{X}+A X+W P\right)+B X+C P+D Q+Q D^{X}\right] .
$$

Further if $h \in T_{\ell}$ then

$$
h^{g}=\left[\begin{array}{lcc}
I & & \\
Y^{-1} A X & I & \\
X^{-1}\left(B X+C P+R Y^{-1} A X\right) & X^{-1} C Y & I
\end{array}\right] .
$$

Certain subgroups of $C_{\ell}$ are of interest:

$$
\begin{aligned}
Z_{\ell} & =\left\{g \in T_{\ell}: P(g)=R(g)=0\right\} \\
J_{\ell} & =\left\{g \in Z_{\ell}: Q(z)=a I, a \in G F(q)\right\} \\
L_{\ell}^{*} & =\left\{g \in C_{\ell}: P(g)=Q(g)=R(g)=0\right\} \\
L_{\ell} & =\left\{g \in L_{\ell}^{*}: \operatorname{det}(X(g))=1\right\} .
\end{aligned}
$$

(4.6) (1) $C_{\ell}=T_{\ell} L_{\ell}^{*}$.

(2) $Z_{\ell}=Z\left(T_{\ell}\right)$.

(3) If $n=2 \ell$ then $T_{\ell}=Z_{\ell}$.

(4) If $n \neq 2 \ell$ then $Z_{\ell}=\Phi\left(T_{\ell}\right)$.

\section{Section 5. Unitary, symplectic and orthogonal groups.}

We continue the hypothesis and notation of Section 4.

Let $\theta$ be an automorphism of $G F(q)$ with $\theta^{2}=1$. A $\theta$-symmetric bilinear form on $V$ is a map (, ) from $V \times V$ to $G F(q)$ such that for each $x, y, z \in V$ and $a \in G F(q)$ 


$$
\begin{gathered}
(x+y, z)=(x, z)+(y, z), \\
(a x, z)=a(x, z), \quad(x, z)^{\theta}=(z, x) .
\end{gathered}
$$

The form is symmetric if $\theta=1$ and hermitian symmetric if $\theta$ is an involution. $v \in V^{\#}$ is singular if $(v, v)=0$. The form is symplectic if it is symmetric and each vector of $V^{\#}$ is singular.

Given a matrix $M=\left(M_{i j}\right)$ over $G F(q)$ let $M^{*}=\left(M_{j i}\right)$ be the transpose of $M$ and $M^{\theta}=\left(M_{i j}^{\theta}\right)$. Define $\pi=* \theta$.

(5.1) Let (, ) be a $\theta$-symmetric form on $V$ and $\left\{x_{i}\right\}$ an ordered basis of $V$. Define $J=\left(J_{i j}\right)$ to be the $n \times n$ matrix with $J_{i j}=\left(x_{i}, x_{j}\right)$. Then $g \in S L(V)$ preserves the form (, ) if and only if

$$
J=g J g^{\pi}
$$

Proof. Straightforward calculation.

For $v \in V$ and $X \subseteq V$ define

$$
\begin{aligned}
v^{\perp} & =\{x \in V:(x, v)=0\} \\
X^{\perp} & =\bigcap_{X} v^{\perp} .
\end{aligned}
$$

The form is said to be nondegenerate if $V^{\perp}=0$. A space $V$ with a nondegenerate hermitian symmetric form is called a unitary space. A space with a nondegenerate symplectic form is called a symplectic space.

It is well known that

(5.2) Let $V$ be a unitary space. Then there exists a basis of $V$ in which $V$ has form $J(V)=I_{n}$.

Define $E_{2 m}$ to be the $2 m \times 2 m$ matrix with 1 in the $(2 i, 2 i-1)$ and $(2 i-1,2 i)$ positions and 0 elsewhere.

(5.3) Let $V$ be a symplectic space. Then $n$ is even and there exists a basis of $V$ in which $V$ has form $J(V)=E_{n}$.

5.2 and 5.3 imply that, up to isomorphism, there is a unique unitary space of dimension $n$ over $G F\left(q^{2}\right)$ and a unique symplectic space of dimension $2 m$ over $G F(q)$. An ordered basis for $V$ in which $V$ has form $I_{n}$ or $E_{n}$, for $V$ unitary or symplectic, will be referred to as a unitary or symplectic basis, respectively.

Denote by $S U(V)$ and $S p(V)$ the subgroup of $S L(V)$ which preserves the corresponding unitary and symplectic form, respectively. $G U(V)$ 
denotes the full group of automorphisms of the unitary space. As $\operatorname{det}(g)=\operatorname{det}\left(g^{*}\right)$ and $E_{n}$ is nonsingular, 5.1 and 5.3 imply $S p(V)$ is the full group of automorphisms of the symplectic space.

A quadratic form on a symplectic space $V$ is a map $Q$ of $V$ to $G F(q)$ such that for each $x, y \in V$ and each $a \in G F(q)$

$$
Q(a x)=a^{2} Q(x), \quad Q(x+y)=Q(x)+Q(y)+(x, y) .
$$

A symplectic space with a quadratic form will be referred to as an orthogonal space.

Suppose $\{u, v\}$ is a symplectic basis for $V$. Define $Q: V \rightarrow G F(q)$ by

$$
Q(a u+b v)=a b .
$$

Then $Q$ is a quadratic form. Denote the 2-dimensional orthogonal space with this form by $D=D_{+}$.

Let $\alpha x^{2}+x+\alpha$ be an irreducible polynomial over $G F(q)$. Then

$$
Q(a u+b v)=\alpha a^{2}+a b+\alpha b^{2}
$$

is a quadratic form on $V$, and the space with this form is denoted by $Q=D_{-}$.

A singular point in an orthogonal space is a 1-dimensional subspace $\langle v\rangle$ with $Q(v)=0$. It is well known that

(5.4) Up to isomorphism, $D$ and $Q$ are the only 2-dimensional orthogonal spaces over $G F(q)$. $Q$ has no singular points. $D$ has exactly two singular points.

Denote by $D^{m} Q^{k}$ the orthogonal sum of $m$ copies of $D$ with $k$ copies of $Q$. It is easy to check

(5.5) $D^{2}$ is isomorphic to $Q^{2}$.

It follows that

(5.6) Up to isomorphism, $D^{m}$ and $D^{m-1} Q$ are the only orthogonal spaces of dimension $2 \mathrm{~m}$.

The orthogonal space $D^{m}$ is said to have sign + and the space $D^{m-1} Q$ sign -. Denote by $O^{*}(V)$ the subgroup of $S p(V)$ preserving the quadratic form on $V$ of $\operatorname{sign} \varepsilon$.

Given an orthogonal space $V$ of $\operatorname{sign} \varepsilon$, an orthogonal basis for $V$ is 
a symplectic basis $\left\{x_{i}\right\}$ such that $Q\left(x_{i}\right)=0$ for $i<n-1$ and

$$
\begin{aligned}
Q\left(a x_{n-1}+b x_{n}\right) & =f_{s}(a, b) \\
f_{+}(a, b)=a b \quad f_{-}(a, b) & =\alpha a^{2}+a b+\alpha b^{2} .
\end{aligned}
$$

By 5.6, $V$ possesses an orthogonal basis.

$$
S L_{n}(q), \quad S U_{n}(q), \quad S p_{n}(q) \text { and } O_{n}^{\mathrm{s}}(q)
$$

denote the special linear, unitary, symplectic and orthogonal groups determined by a space of dimension $n$ over $G F(q)$, or $G F\left(q^{2}\right)$ in the unitary case. $S L_{n}(q)$ has a center of order $(q-1, n)$ and $S U_{n}(q)$ has a center of order $(q+1, n) . \quad L_{n}(q)$ and $U_{n}(q)$ denote the factor groups over these centers. $\Omega_{n}^{s}(q)$ denotes the commutator group of $O_{n}^{s}(q)$. As $q$ is even $\Omega_{n}^{s}(q)=S O^{s}(n, q)$.

\section{Section 6. Involutions in the unitary group.}

We continue the hypothesis and notation of sections 4 and 5 .

(6.1) Let $a$ and $b$ be involutions in $S U(V)$. Then the following are equivalent.

(1) $a$ is conjugate to $b$ in $S U(V)$.

(2) $a$ is conjugate to $b$ in $S L(V)$.

(3) $a$ and $b$ have the same rank.

Proof. See [25], page 34.

(6.2) Let $t$ be an involution in $S U(V)$ of rank $\ell$. Then there exists a basis for $V$ in which $t=j_{\ell}$ is in Suzuki form and $V$ has unitary form

$$
J(V)=\left[\begin{array}{lll} 
& & I_{\ell} \\
& I_{n-2 \ell} &
\end{array}\right] .
$$

Further $g \in C_{\ell}$ is in $S U(V)$ if and only if

$$
\begin{gathered}
X^{\pi}=X^{-1} \quad Y^{\pi}=Y^{-1} \quad Y R^{\pi}=P X^{\pi} \\
X Q^{\pi}+R R^{\pi}+Q X^{\pi}=0 .
\end{gathered}
$$

The map $g \rightarrow(X(g), Y(g))$ is a homomorphism of $C=C(t) \cap S U(V)$ into $G U_{\ell}(q) \times G U_{n-2 \ell}(q)$ with the image containing $S U_{\ell}(q) \times S U_{n-2 \ell}(q)$, and covering both factors if $n \neq 2 \ell$. The kernel is $T_{\ell} \cap C=O_{2}(C)$. 
Proof. Let $\left\{x_{i}\right\}$ be a unitary basis for $V$. Define $s \in S L(V)$ by

$$
\begin{aligned}
& x_{i}^{s}=x_{n-\ell+i} \quad 1 \leq i \leq \ell \\
& x_{i}^{s}=x_{i} \quad \ell<i \leq n-\ell \text {. }
\end{aligned}
$$

Then $s$ has rank $\ell$ and by 5.1, $s \in S U(V)$. Hence by 6.1, we may take $t=s$. Choose $\alpha \in F_{q^{2}}$ such that $\alpha+\alpha^{\theta}=1$ and let $\beta=1+\alpha$. Define

$$
\begin{aligned}
& y_{i}=x_{i}+x_{i}^{t} \quad 1 \leq i \leq \ell \\
& y_{i}=x_{i} \quad \ell<i \leq n-\ell \\
& y_{i}=\alpha x_{i-n+\ell}+\beta x_{i} \quad n-\ell \leq i \leq n .
\end{aligned}
$$

Then $\left\{y_{i}\right\}$ is the desired basis. Calculating using 5.1, we determine the conditions on $g$. This together with 4.3 yields the remaining conclusions.

\section{Section 7. Involutions in the symplectic groups.}

In this section we assume $V$ to be a symplectic space and $t$ an involution in $S p(V)$. It is well known that

(7.1) Let $U \leq V$. Then $\operatorname{dim}(V)=\operatorname{dim}(U)+\operatorname{dim}\left(U^{\perp}\right)$. Therefore if $U$ is non-degenerate then $V$ is the orthogonal sum of $U$ and $U^{\perp}$.

Given an involution $t \in S p(V)$ define

$$
V(t)=\left\{v \in V:\left(v, v^{t}\right)=0\right\}
$$

$V(t)$ is the kernel of the additive map $v \rightarrow\left(v, v^{t}\right)$, so

(7.2) $V(t)$ is a subspace of $V$ of codimension at most 1 with $V(t)^{\perp} \leq V(t)$.

Given subspaces $U$ and $W$ of $V$ with $U \cap W=0$ and $(U, W)=0$, write $\langle U, W\rangle=U \oplus W$. That is $U \oplus W$ is the orthogonal sum of $U$ and $W$.

(7.3) Assume $V=V(t)$. Then

$$
V=\bigoplus_{i=1}^{m} V_{i} \oplus W
$$

where $[W, t]=0$ and each $V_{i}$ is a 4-dimensional symplectic space with symplectic basis $\left\{y_{i j}\right\}$ such that $y_{i 1}^{t}=y_{i 3}$ and $y_{i 2}^{t}=y_{i 4}$. In particular $t$ has even rank $\ell=2 m$. 
Proof. Choose $u \in V$ with $u^{t} \notin\langle u\rangle$. Set $u=y_{1}$ and $u^{t}=y_{3}$. Let $v \in\left(u^{t}\right)^{\perp}-u^{\perp}$. Then $v^{t} \in u^{\perp}-\left(u^{t}\right)^{\perp}$, so $v \neq v^{t}$. By choosing a suitable multiple of $v$ we may take $(u, v)=1$. Set $y_{2}=v$ and $y_{4}=v^{t}$. Then $V_{1}=\left\langle y_{i}: 1 \leq i \leq 4\right\rangle$ has the properties claimed above. $V_{1}$ is nondegenerate, so by $7.1, V=V_{1} \oplus V_{1}^{\perp}$, with $V_{1}^{\perp}$ a $t$-invariant symplectic space. Hence the result follows by induction on the dimension of $V$.

The following lemma is immediate.

(7.4) Assume $u \in V$ with $\left(u, u^{t}\right) \neq 0$. Then for $a^{2}=\left(u, u^{t}\right)^{-1}, x_{1}=$ au and $x_{2}=x_{1}^{t}$ is a symplectic basis for $\left\langle u, u^{t}\right\rangle$.

(7.5) Assume $U=V(t) \neq V$ and set $X=U^{\perp}$. Let $\ell$ be the rank of $t$. Then either

(1) $\quad$ is odd, $[V, t]=X \oplus[U, t]$ and $V=Y \oplus Y^{\perp}$ where $Y$ has symplectic basis $\left\{y, y^{t}\right\}$ and $[Y, t]=X$.

(2) $\ell$ is even and $V=Y \oplus Y^{\perp}$ for some $Y$ with symplectic basis $\left\{y_{i}: 1 \leq i \leq 4\right\}$ such that $y_{1}^{t}=y_{2}, y_{3}^{t}=y_{4}$. Further $[U, t]=\left[Y^{\perp}, t\right] \oplus X$ has codimension 1 in $[V, t]$ and $X=Y(t)^{\perp}$.

Proof. Let $P$ be maximal with respect to $P=\oplus P_{i}$ where $P_{i}$ has a symplectic basis $\left\{x_{i}, x_{i}^{t}\right\}$. By 7.1, $V=P \oplus P^{\perp}$. By 7.4 and maximality of $P, P^{\perp} \leq U . \quad[V, t]=[P, t] \oplus\left[P^{\perp}, t\right]$, so $\ell=\operatorname{dim}([P, t])+\operatorname{dim}\left(\left[P^{\perp}, t\right]\right)=$ $a+b$. By 7.3, $b$ is even, so $\ell \equiv a \bmod 2$. Further $U=P^{\perp} \oplus P(t)$ and

$$
P(t)=\left\langle x_{i}+x_{j}, x_{i}+x_{i}^{t}: 1 \leq i \leq a, 1 \leq j \leq a\right\rangle .
$$

Hence $x=\sum_{i}\left(x_{i}+x_{i}^{t}\right)$ is a generator of $X$.

Assume $\ell$ is odd. Then $a$ is odd, so setting $y=\sum_{i} x_{i},\left\{y, y^{t}\right\}$ is a symplectic basis for $Y=\left\langle y, y^{t}\right\rangle$ and $[y, t]=X$. By 7.1, $V=Y \oplus Y^{\perp}$. Then $Y^{\perp} \leq X^{\perp} \leq U$ and $[V, t]=[Y, t] \oplus\left[Y^{\perp}, t\right]=X \oplus[U, t]$.

So assume $\ell$ is even. Let $y_{3}=\sum_{i \neq 1} x_{i}$ and $y_{4}=y_{3}^{t}$. $\quad$ is even so $a-1$ is odd and hence $\left(y_{3}, y_{4}\right)=1$. Let $x_{1}=y_{1}$ and $x_{1}^{t}=y_{2}$. Then $Y=$ $\left\langle y_{i}: 1 \leq i \leq 4\right\rangle$ is as claimed. By 7.1, $V=Y \oplus Y^{\perp}$. Then $U=Y^{\perp} \oplus Y(t)$ and $Y(t)=\left\langle y_{i}+y_{j}: 1 \leq i \leq j \leq 4\right\rangle$. So $[U, t]=\left[Y^{\perp}, t\right] \oplus[Y(t), t]=\left[Y^{\perp}, t\right]$ $\left(\oplus X\right.$ has codimension 1 in $[V, t]=\left[Y^{\perp}, t\right] \oplus[Y, t]$.

(7.6) Let $\ell$ be the rank of $t$. Then there exists a basis $\left\{x_{i}\right\}$ of $V$ with form 


$$
J=\left[\begin{array}{lll} 
& & F \\
F & E_{n-2 \ell} &
\end{array}\right]
$$

in which $t=j_{\ell}$ and exactly one of the following holds:

(1) $\ell$ is even, $V=V(t)$ and $F=E_{\ell}$.

(2) $\ell$ is odd, $V(t)=\left\langle x_{i}: i \neq n-\ell+1\right\rangle, V(t)^{\perp}=\left\langle x_{1}\right\rangle$

$$
\begin{gathered}
{[V(t), t]^{\perp}=\left\langle x_{i}: 1 \leq i \leq n-\ell+1\right\rangle} \\
{[V(t), t]=\left\langle x_{i}: 1 \leq i \leq \ell\right\rangle} \\
F=\left[\begin{array}{ll}
1 & \\
& E_{\ell-1}
\end{array}\right] .
\end{gathered}
$$

(3) $\quad$ is even, $V(t)=\left\langle x_{i}: 1 \leq i\langle n\rangle, V(t)^{\perp}=\left\langle x_{1}\right\rangle\right.$

$$
\begin{aligned}
{[V(t), t]^{\perp} } & =\left\langle x_{i}: 1 \leq i \leq n-\ell+1\right\rangle \\
{[V(t), t] } & =\left\langle x_{i}: 1 \leq i<\ell\right\rangle \\
F & =\left[\begin{array}{rrr} 
& & 1 \\
& E_{\ell-2} & \\
1 & & 1
\end{array}\right] .
\end{aligned}
$$

Proof. Assume first $V=V(t)$. Then define $V_{i}, y_{i j}$, and $W, 1 \leq i \leq$ $\ell / 2,1 \leq j \leq 4$, as in 7.3. Define

$$
\begin{aligned}
& x_{2 i-1}=y_{i 1}+y_{i 3} \\
& x_{2 i}=y_{i 2}+y_{i 4} \\
& x_{n-\ell+2 i-1}=y_{i 1} \\
& x_{n-\ell+2 i}=y_{i 2}
\end{aligned}
$$

and let $\left\{x_{\ell+i}: 1 \leq i \leq n-2 \ell\right\}$ be a symplectic basis for $W$.

Assume next that $V(t) \neq V$. Choose $Y$ and its symplectic basis as in 7.5. If $\ell$ is odd let $x_{1}=y, x_{n-\ell+1}=y^{t}$, and choose $\left\{x_{i}: 1 \neq i \neq n-\ell+1\right\}$ to be a basis for $Y^{\perp}=Y^{\perp}(t)$ as in the last paragraph. If $\ell$ is even define

$$
\begin{gathered}
x_{1}=\sum_{i} y_{i} \quad x_{\ell}=y_{3}+y_{4} \\
x_{n-\ell+1}=y_{1}+y_{4} \quad x_{n}=y_{4} .
\end{gathered}
$$

Choose the remaining basis vectors as in the last paragraph.

We shall say an involution $t \in S p(V)$ is in symplectic Suzuki form if the basis is chosen as in 7.6. Denote by $a_{\ell}, b_{\ell}$, and $c_{\ell}$ the Suzuki 
forms in (1), (2), and (3) of 7.6, respectively. It follows from 7.6 that (7.7) Let $t$ and $s$ be involutions in $S p(V)$. Then the following are equivalent :

(1) $t$ is conjugate to $s$ in $S p(V)$.

(2) $t$ and $s$ have the same symplectic Suzuki form.

(3) $t$ and $s$ have the same rank $\ell$, and if $\ell$ is even then $V(t)$ and $V(s)$ have the same dimension.

(7.8) Let $F=F^{*}$ be an $\ell \times \ell$ matrix and

$$
J=\left[\begin{array}{lll} 
& & F \\
F & E_{n-2 \ell} &
\end{array}\right] .
$$

Let $g \in C_{\ell}$. Then $g J g^{*}=J$ if and only if

$$
\begin{gathered}
X F X^{*}=F \quad Y E Y^{*}=E \quad Y E R^{*}=P F X^{*} \\
0=X F Q^{*}+R E R^{*}+Q F X^{*} .
\end{gathered}
$$

(7.9) Let $t=a_{\ell}$ be in Suzuki form, $g \in C_{\ell}$, and $E=E_{\ell}$ or $E_{n-2 \ell}$. Then $g \in S p(V)$ if and only if

$$
\begin{gathered}
X E X^{*}=E \quad Y E Y^{*}=E \quad Y E R^{*}=P E X^{*} \\
0=X E Q^{*}+R E R^{*}+Q E X^{*} .
\end{gathered}
$$

The map $g \rightarrow(X(g), Y(g))$ is a homomorphism of $C=C_{\ell} \cap S p(V)$ onto $S p_{\ell}(q) \times S p_{n-2 \ell}(q)$ with kernel $T_{\ell} \cap S p(V)=O_{2}(C)$.

Proof. 4.3,7.6, and 7.8.

(7.10) Let $t=b_{\ell}$ be in Suzuki form, let $g \in C_{\ell}$, and $E=E_{\ell-1}$ or $E_{n-2 \ell}$. Then

(1) $g \in S p(V)$ if and only if

$$
\begin{gathered}
X=\left[\begin{array}{ll}
1 & \\
& W
\end{array}\right] \quad R=\left[\begin{array}{l}
\alpha \\
A
\end{array}\right] \quad P=\left[\begin{array}{ll}
Y \alpha^{*} & Y E A^{*} E W
\end{array}\right] \\
Q=\left[\begin{array}{ll}
b & B \\
\gamma & L
\end{array}\right]
\end{gathered}
$$

with $\alpha, \beta$ and $\gamma^{*}$ row vectors and

$$
\begin{gathered}
W E W^{*}=E \quad \alpha E A^{*}=\gamma^{*}+\beta E W^{*} \\
Y E Y^{*}=E \quad L E W^{*}+W E L^{*}=A E A^{*}
\end{gathered}
$$


(2) The map $g \rightarrow(W(g), Y(g))$ is a homomorphism of $C=C_{\ell} \cap S p(V)$ onto $S p_{\ell-1}(q) \times S p_{n-2 \ell}(q)$ with kernel $T_{\ell} \cap C=O_{2}(C)$.

(7.11). Let $t=c_{\ell}$ be in Suzuki form, let $g \in C_{\ell}, E=E_{\ell-2}$ or $E_{n-2 \ell}$, and

$$
F=\left[\begin{array}{lll} 
& & 1 \\
& E_{\ell-2} & \\
1 & & 1
\end{array}\right]
$$

Then

(1) $g \in S p(V)$ if and only if

$$
\begin{gathered}
X=\left[\begin{array}{ccc}
1 & & \\
W E \alpha^{*} & W & \\
x & \alpha & 1
\end{array}\right] \quad R=\left[\begin{array}{l}
\beta \\
A \\
\gamma
\end{array}\right] \\
Q=\left[\begin{array}{lll}
r & \rho & z \\
\mu & L & \eta \\
y & \xi & s
\end{array}\right]
\end{gathered}
$$

where $\beta, \gamma, \rho, \xi, \mu^{*}$, and $\eta^{*}$ are row vectors and

$$
\begin{aligned}
& W E W^{*}=E \quad Y E Y^{*}=E \quad P=Y E R^{*} F^{-1} X \\
& 0=X F Q^{*}+R E R^{*}+Q F X^{*} \text {. }
\end{aligned}
$$

(2) The map $g \rightarrow(W(g), Y(g))$ is a homomorphism of $C=C_{\ell} \cap S p(V)$ onto $S p_{\ell-2}(q) \times S p_{n-2 \ell}(q)$ with kernel $\mathrm{O}_{2}(C)$.

(7.12) Assume the hypothesis of 7.11. Then

(1) If $g \in Z_{\ell}$ then $g \in S p(V)$ if and only if

$$
\begin{aligned}
& r+s+z=0 \quad L E=E L^{*} \\
& \eta=E \rho^{*} \quad \xi E=\mu^{*}+\eta^{*} .
\end{aligned}
$$

(2) If $g \in Z_{\ell} L_{\ell}$ with $\alpha=\mu=\rho=\eta=\xi=0$ then $g \in S p(V)$ if and only if

$$
\begin{aligned}
& Y E Y^{*}=E \quad W E L^{*}=L E W^{*} \\
& W E W^{*}=E \quad r+z+s+x z=0 .
\end{aligned}
$$

(7.13) Let $Q$ and $X$ be as in 7.11. Then

(1) If $\alpha=0$ and $W=I$ then 


$$
Q^{x}=\left[\begin{array}{ccc}
r+z x & \rho & z \\
\mu+x \eta & L & \eta \\
y+(r+s+z x) x & \xi+\rho x & s+z x
\end{array}\right] .
$$

(2) If $\mu=\eta=\rho=\xi=0, W=I$ and $L=a I$ then

$$
Q^{x}=\left[\begin{array}{ccc}
r+z x & z \alpha & z \\
(r+z x+a) E \alpha^{*} & L+z E \alpha^{*} \alpha & z E \alpha^{*} \\
y+(r+s+z x) x & \alpha(a+s+x z) & x z+s
\end{array}\right] .
$$

These last three lemmas follow from straight forward calculations.

\section{Section 8. Involutions in the orthogonal groups.}

In this section assume that $V$ is an orthogonal space of $\operatorname{sign} \varepsilon$. It is well known that

(8.1) (1) $O_{2}^{s}(q)$ is dihedral of order $2(q-\varepsilon)$.

(2) $O_{4}^{+}(q)$ is the wreath product of $L_{2}(q)$ by $Z_{2}$.

(3) $O_{4}^{-}(q)$ is the split extension of $L_{2}\left(q^{2}\right)$ by a field automorphism of order 2.

(4) $O_{6}^{+}(q)$ is the split extension of $L_{4}(q)$ by a graph automorphism.

(5) $O_{6}^{-}(q)$ is the split extension of $U_{4}(q)$ by a graph automorphism.

Given the isomorphisms in 8.1 we assume $n \geq 8$ in this section, although most of the discussion remains valid without this restriction.

(8.2) Let $t$ be an involution in $O^{\circ}(V)$ of type $a_{\ell}$. Then there exists a basis $\left\{x_{i}\right\}$ of $V$ in which $t$ is in its symplectic Suzuki form, $Q\left(x_{i}\right)=0$ for $1 \leq i \leq \ell$ and $n-\ell<i \leq n,\left\{x_{i}: \ell<i \leq n-\ell\right\}$ is an orthogonal basis for the space $W$ it generates, and $W$ has sign $\varepsilon$.

Proof. Choose $\left\{x_{i}\right\}$ so that $t$ is in Suzuki form. For $1 \leq i \leq \ell$ define

$$
U_{i}=\left\langle x_{n-\ell+2 i-1}, x_{n-\ell+2 i}\right\rangle .
$$

Then $V_{i}=U_{i} \oplus U_{i}^{t} \cong U_{i} \oplus U_{i}$, so by 5.5, $V_{i}$ is isomorphic to $D^{2}$. Thus choosing the basis vectors $\left\{y_{i j}\right\}$ for $V_{i}$ in 7.3 to be an orthogonal basis for $V_{i}$, we have $Q\left(x_{j}\right)=0$. Further as $V$ is the orthogonal sum of $W$ and the spaces $V_{i}$, and each $V_{i}$ has $\operatorname{sign}+, \varepsilon=\operatorname{sgn}(V)=\operatorname{sgn}(W)$. Hence we need only choose the basis vectors $x_{j}$ in $W$ to form an orthogonal basis for $W$. 
(8.3) Let $t \in O^{*}(V)$ be the type $b_{\ell}$. Then there is a basis $\left\{x_{i}\right\}$ of $V$ in which $t$ is in symplectic Suzuki form, $Q\left(x_{1}\right)=1, X=\left\langle x_{1}, x_{n-\ell+1}\right\rangle$ has $\operatorname{sign} \varepsilon ; Q\left(x_{i}\right)=0$ for $1<i \leq \ell$ and $n-\ell+1<i \leq n,\left\{x_{i}: \ell<i \leq n-\ell\right\}$ is an orthogonal basis for the space $W$ it generates, and $W$ has sign + .

Proof. Choose $\left\{x_{i}\right\}$ so that $t$ is in Suzuki form. Define

$$
U=\left\langle x_{i}: 1<i \leq \ell, n-\ell+1<i \leq n\right\rangle .
$$

Considering $t$ restricted to $U, 8.2$ implies $U$ has sign + and we may choose the basis vectors $x_{j}$ in $U$ to be singular. $t$ restricted to $X$ is the transvection with center $x_{1}$, so $Q\left(x_{1}\right)=1$.

Suppose $W$ has sign -. We may assume $Y=\left\langle x_{\ell+1}, x_{\ell+2}\right\rangle \cong Q$. Let $a^{2}=Q\left(x_{\ell+1}\right), x_{\ell+1}^{\prime}=a x_{1}+x_{\ell+1}$, and $x_{n-\ell+1}^{\prime}=x_{n-\ell+1}+a x_{\ell+2}$. Then $Q\left(x_{\ell+1}^{\prime}\right)=0$ and $\left\{x_{1}, x_{n-\ell+1}^{\prime}, x_{\ell+1}^{\prime}, x_{\ell+2}\right\}$ is a symplectic basis for $X+Y$. As $Q\left(x_{\ell+1}^{\prime}\right)=0$, $Y^{\prime}=\left\langle x_{\ell+1}^{\prime}, x_{\ell+2}\right\rangle \cong D$, so with this change of basis $W$ has sign $\varepsilon$. Hence $\operatorname{sgn}(X)=\operatorname{sgn}(V)=\varepsilon$.

The proof of the next lemma is similar to that of 8.2 and 8.3 , and is omitted.

(8.4) Let $t \in O^{*}(V)$ be of type $c_{\ell}$. Then there is a basis $\left\{x_{i}\right\}$ of $V$ in which $t$ is in Suzuki form, $Y=\left\langle x_{1}, x_{\ell}, x_{n-\ell+1}, x_{n}\right\rangle$ has sign $\varepsilon, Q\left(x_{i}\right)=0$ for $1<i<\ell$ and $n-\ell+1<i<n,\left\{x_{i}: \ell<i \leq n-\ell\right\}$ is an orthogonal basis for the space $W$ it generates, and $W$ has sign + . Also

$$
Q\left(a x_{1}+b x_{\ell}+c x_{n-\ell+1}+d x_{n}\right)=a d+b(b+c+d)+\left(c^{2}+d^{2}\right) Q\left(x_{n}\right) .
$$

We shall say an involution $t$ in $O^{*}(V)$ is in orthogonal Suzuki form if the basis for $V$ is chosen as in 8.2-8.4 for the suitable type of $t$. In particular if $t$ is in its orthogonal Suzuki form it is also in symplectic Suzuki form. Therefore

(8.5) Let $t$ and $s$ be involutions in $O^{*}(V)$. Then the following are equivalent :

(1) $t$ is conjugate to $s$ in $O^{*}(V)$.

(2) $t$ is conjugate to $s$ in $S p(V)$.

(3) $t$ and $s$ have the same (orthogonal or symplectic) Suzuki form.

(8.6) Let $t=a_{\ell}$ be in orthogonal Suzuki form and $g \in C_{\ell} \cap S p(V)$. Then (1) $g \in O^{*}(V)$ if and only if $Y(g) \in O_{n-2 \ell}^{*}(q)$ and 


$$
\sum_{j=1}^{\ell / 2} g_{i(2 j-1)} g_{i(n-\ell+2 j)}+g_{i(2 j)} g_{i(n-\ell+2 j-1)}=\sum_{j=1}^{(n-2 \ell) / 2} g_{i(\ell+2 j-1)} g_{i(\ell+2 j)}
$$

(2) The map $g \rightarrow(X(g), Y(g))$ is a homomorphism of $C=C_{\ell} \cap O^{*}(V)$ onto $S p_{\ell}(q) \times O_{n-2 \ell}^{s}(q)$ with kernel $T_{\ell} \cap C=O_{2}(C)$.

Proof. Let $G=S p(V), H=O^{\circ}(V)$, and $g \in C_{\ell} \cap G$. Then $g \in H$ if and only if $Q\left(x_{i}^{q}\right)=Q\left(x_{i}\right)$ for each $i$. Define

$$
\begin{aligned}
U & =\left\langle x_{i}: 1 \leq i \leq \ell, n-\ell\langle i \leq n\rangle\right. \\
W & =\left\langle x_{i}: \ell<i \leq n-\ell\right\rangle .
\end{aligned}
$$

Then $V=U \oplus W$ and from the form of $J(V)$ in 7.6 and the value of the quadratic form $Q$ on $\left\{x_{i}\right\}$ given in 8.2 we determine that for $u+w=$ $\sum a_{i} x_{i}$

$$
Q(u+w)=\sum_{i=1}^{\ell / 2} a_{2 j-1} a_{n-\ell+2 j}+a_{2 j} a_{n-\ell+2 j-1}+Q(w)
$$

and $Q$ restricted to $W$ has sign $\varepsilon$.

Hence for $1 \leq i \leq \ell, Q\left(x_{i}\right)=0$ and

$$
Q\left(x_{i}^{g}\right)=Q\left(\sum g_{i j} x_{j}\right)=\sum_{i=1}^{\ell} g_{i j} g_{i(n-\ell+j)}=0
$$

since $g_{i j}=0$ for $j>\ell$. That is $Q\left(x_{i}\right)=Q\left(x_{i}^{q}\right)$ for $i \leq \ell$. Similarly for $x_{i} \in W, Q\left(x_{i}^{g}\right)=Q\left(x_{i} Y(g)\right)$, so $Q\left(x_{i}\right)=Q\left(x_{i}^{g}\right)$ if and only if $Y(g) \in O^{s}(W)=$ $O_{n-2 \ell}^{s}(q)$. Finally if $Q(g)=P(g)=0$, then for $i \leq \ell, Q\left(x_{n-\ell+i}\right)=0=$ $Q\left(x_{n-\ell+1}^{g}\right)$. Therefore 7.9 yields the result.

(8.7) Let $t=b_{\ell}$ be in orthogonal Suzuki form. Then the map $g \rightarrow$ $(W(g), Y(g))$ is a homomorphism of $C=C_{\ell} \cap O^{*}(V)$ onto $S p_{\ell-1}(q) \times S p_{n-2 \ell}(q)$ with kernel $T_{\ell} \cap C=O_{2}(C)$.

Proof. The proof goes as in 8.6. The only problem is to show that if $\varphi: g \rightarrow(W(g), Y(g))$ then $C \varphi$ covers $S p_{\ell-1}(q) \times S p_{n-2 \ell}(q)$. Proceeding as in 8.6, $C \varphi$ covers $S p_{\ell-1}(q) \times O_{n-2 \ell}^{+}(q)$. Let $U=\left\langle x_{i}: \ell\langle i \leq n-\ell\rangle\right.$ and $Y$ the transvection in $S p(U)$ with center $x_{\ell+1}$. Then $Y \notin O^{+}(U)$. Further $O^{+}(U)$ is maximal in $S p(U)$, so $S p(U)=\left\langle Y, O^{+}(U)\right\rangle$, and it suffices to exhibit $g \in C$ with $Y(g)=Y$. Define $g$ by 


$$
g=\left(\begin{array}{lllll}
1 & & & & \\
& I & & & \\
\alpha & & Y & & \\
& & \beta & 1 & \\
& & & & I
\end{array}\right)
$$

where $\alpha^{*}=(0,1,0, \cdots)$ and $\beta=(1,0,0, \cdots)$.

(8.8) Let $t=c_{\ell}$ be in orthogonal Suzuki form and $g \in C_{\ell} \cap S p(V)$. Given a matrix $A=\left(a_{i j}\right)$ let $A_{i}$ be the ith row vector of $A$ and define

$$
\begin{aligned}
Q\left(A_{i}\right) & =\sum_{j} a_{i(2 j-1)} a_{i(2 j)} \\
\left(A_{i}, B_{i}\right) & =A_{i} E B_{i}^{*} .
\end{aligned}
$$

Then

(1) $g \in O^{*}(V)$ if and only if (i)-(iv) hold with notation for $g$ as in 7.11 and 7.12 .

(i) $\tau_{i}^{2}=Q\left(Y_{i}\right)$ for $1 \leq i \leq n-2 \ell$.

(ii) $z(z+1)=Q(\beta)$.

(iii) $\eta_{i}\left[\eta_{i}+\left(W E \alpha^{*}\right)_{i}\right]+\left(W E \alpha^{*}\right)_{i}^{2} Q\left(x_{n}\right)+\left(L_{i}, W_{i}\right)=0$.

(iv) $y+s(s+x+1)+x^{2} Q\left(x_{n}\right)+(\xi, \alpha)=0$.

(2) The map $g \rightarrow(W(g), Y(g))$ is a homomorphism of $C=O^{*}(V) \cap C_{\ell}$ onto $S p_{\ell-2}(q) \times S p_{n-2 \ell}(q)$ with kernel $O_{2}(C)$.

Proof. The proof goes as in 8.6 and 8.7. We exhibit an element $g \in C$ such that $Y(g)=Y$ is the transvection with center $x_{\ell+1}$ :

$$
g=\left(\begin{array}{lllllll}
1 & & & & & \\
& I & & & & \\
& & 1 & & & \\
\tau & & \tau & & & \\
& & & Y & & \\
& & & \beta & 1 & \\
& & & & & I
\end{array}\right)
$$

where $\tau^{*}=(0,1,0, \cdots)$ and $\beta=(1,0,0, \cdots)$.

(8.9) Let $t=c_{\ell}$ be in orthogonal Suzuki form and $g \in C_{\ell}$ with notation as in 7.11 and 7.12. Then

(1) If $g \in Z_{\ell}$ then $g \in O^{*}(V)$ if and only if $z=0$ or $1, y=s^{2}+s, r+s$ $+z=0, \eta=E \rho^{*}, \xi E=\mu^{*}+\eta^{*}, L^{E}=L^{*}$, and

$$
\eta=\left(L_{12}, L_{21}, L_{34}, L_{43}, \cdots\right)^{*} \text {. }
$$


(2) If $g \in Z_{\ell} L_{\ell}$ with $\alpha=\mu=\rho=\eta=\xi=0$ then $g \in O^{\circ}(V)$ if and only if $W E W^{*}=E, Y \in O_{n-2 \ell}^{+}(q), W E L^{*}=L E W^{*}, r+z+s+x z=0, y+s(s+x+1)$ $=x Q\left(x_{n}\right), z=0$ or 1 , and $\left(L_{i}, W_{i}\right)=0$ for each $1 \leq i \leq \ell-2$.

Proof. Calculate.

(8.10) The commutator group $\Omega^{*}$ of $O^{*}$ is a simple subgroup of index 2 in $O^{\star}$. $t \in \Omega^{\star}$ if and only if $t$ is of type a or $c$.

Proof. The first statement is well known. The transvections in $O^{8}$ are not contained in $\Omega^{s}$. Further if $\ell$ is the rank of $t$ then $t$ is the product of $m$ transvections where $\ell \equiv m \bmod 2$.

(8.11) Let $t=c_{2}$ and $z$ are involutions in $Z_{\ell} \cap \Omega^{*}(V)$. Then

$$
Q(z)=\left[\begin{array}{cc}
r & 0 \\
r(r+1) & r
\end{array}\right]
$$

Proof. By 8.9 if $a$ is an involution in $Z_{\ell} \cap O^{*}(V)$ then

$$
Q(\alpha)=\left[\begin{array}{cc}
s+z & z \\
s(s+1) & s
\end{array}\right]
$$

with $z=0$ or 1 . But if $z=1$ then $a$ has $\operatorname{rank} 1$, so $a \notin \Omega^{\star}(V)$.

(8.12) Let $G=\Omega_{n}^{\varepsilon}(q)$ and $t$ an involution of $G$ in Suzuki form. Then (1) $t^{G}$ is a conjugacy class in $O_{n}^{*}(q)$ unless $n=2 \ell, \varepsilon=+$, and $t=a_{\ell}$.

(2) Let $n=2 \ell, \varepsilon=+$, and $t=a_{\ell}$. Then the class of $t$ in $O_{n}^{\varepsilon}(q)$ splits into two classes in $G$. If $s$ is of type $a_{\ell}$ then $s \in t^{G}$ exactly when $\operatorname{dim}([V, s] \cap[V, t])$ is even. Further $C_{G}(t)=\left\langle t^{G} \cap C_{G}(t)\right\rangle O_{2}\left(C_{G}(t)\right)$.

Proof. Unless $t=a_{\ell}, n=2 \ell$, and $\varepsilon=+, t$ centralizes a transvection, so by $8.10, t^{G}$ is a conjugacy class of $O_{n}^{\varepsilon}$. So assume $n=2 \ell, \varepsilon=+$, and $t=a_{\ell}$. Then, under the action of $G$, there are two classes of totally isotropic $\ell$-dimensional subspaces of $V$. Moreover if $U$ and $W$ are two such subspaces then $U$ is conjugate to $W$ under $G$ exactly when $\operatorname{dim}(U \cap W)$ is even. As $[V, t]$ is such a subspace, the first two remarks of (2) follow.

If $\ell \equiv 0 \bmod 4$ then the involution $s \in L$ with $X(s)=E_{\ell}$ is in $t^{G}$, since $\operatorname{dim}([V, t] \cap[V, s])=\ell / 2$ is even. Similarly if $\ell \equiv 2 \bmod 4$ then the involution $r \in C_{\ell}$ with 


$$
X(r)=\left[\begin{array}{ll}
E_{\ell-2} & \\
& I_{2}
\end{array}\right] \quad Q(r)=\left[\begin{array}{ll} 
& \\
& I_{2}
\end{array}\right]
$$

is in $t^{G}$. Also $C(t) / O_{2}(C(t)) \cong L$ is simple unless $q=2$ and $n=4$, in which case $\left\langle s^{L}\right\rangle=L$. So the last part of (2) follows.

\section{Section 9. Two lemmas.}

In this section we prove two lemmas which help describe the action of the centralizer $C$, of an involution in the classical groups, on $\mathrm{O}_{2}(C)$.

Let $G$ be the subgroup of $S L_{\ell}(q), S U_{\ell}(q)$, or $S p_{\ell}(q)$ generated by all transvections, or a subgroup of $O_{\ell}^{s}(q)$ of index at most 2 . Here $q$ is a power of $2, \ell>1$, and $\ell \geq 4$ if $G \leq O_{\ell}^{+}(q)$. Consider $G$ as a matrix group with respect to a natural basis $\left\{x_{i}\right\}$ for the linear space $V$ corresponding to $G$.

Let $M$ be the ring of all $n \times \ell$ matrices over the corresponding field, i.e. $G F(q)$ unless $G \leq S U(q)$, in which case the field is $G F\left(q^{2}\right)$.

(9.1) Let $G$ act by right multiplication on $M$. Then $C_{M}(G)=0$ and $G$ has no orbits of length 2.

Proof. If $P \in M, g \in G$, and $P g=P$, then $g$ fixes each of the row vectors of $P$. Consequently $C_{M}(G)=0$. If $P$ is in an orbit of length 2, then $C_{G}(P)=H \leq G$ has index 2 in $G$ and it is easy to check that this forces $P=0$, a contradiction.

(9.2) Let $n=\ell$ and exclude the case $G \leq O_{\ell}^{:}(q)$. Let $G$ act by conjugation on $M$. Then the centralizer in $M$ of $G$ is the set of scalar matrices and if $N$ is a G-invariant 4-group of $M$ containing $I$ with $[G, N] \neq 0$, then one of the following holds:

(1) $G=L_{2}(2)$ and $N$ consists of

$$
\left[\begin{array}{ll}
0 & 0 \\
0 & 0
\end{array}\right],\left[\begin{array}{ll}
1 & 0 \\
0 & 1
\end{array}\right],\left[\begin{array}{ll}
1 & 1 \\
1 & 0
\end{array}\right],\left[\begin{array}{ll}
0 & 1 \\
1 & 1
\end{array}\right] .
$$

(2) $G=U_{2}(2)$ and choosing a to be a generator of $G F(4), N$ consists of either

$$
\left[\begin{array}{ll}
0 & 0 \\
0 & 0
\end{array}\right],\left[\begin{array}{ll}
1 & 0 \\
0 & 1
\end{array}\right],\left[\begin{array}{cc}
a & 0 \\
0 & a^{2}
\end{array}\right],\left[\begin{array}{cc}
a^{2} & 0 \\
0 & a
\end{array}\right]
$$

or 
(ii)

$\left[\begin{array}{ll}0 & 0 \\ 0 & 0\end{array}\right],\left[\begin{array}{ll}1 & 0 \\ 0 & 1\end{array}\right],\left[\begin{array}{ll}1 & 0 \\ 0 & 0\end{array}\right],\left[\begin{array}{ll}0 & 0 \\ 0 & 1\end{array}\right]$

(3) $\quad G \leq S U_{3}(2)$ and $S U_{3}(2)$ does not act on $N$.

Proof. $C_{M}(G)=\langle\alpha I\rangle$ is well-known and equivalent to the fact that $G$ is absolutely irreducible on $V$. Assume that $N$ is a 4-group as described. Then $G$ has an orbit of length 2 on $N$ so $q=2$ and $G=L_{2}(2), U_{2}(2)$, $S p(4,2)$, or the subgroup of $S U_{3}(2)$ generated by transvectors.

If $G=S p(4,2)$, then $G^{\prime} \leq C(N)$, contradicting the fact that $G^{\prime}$ is absolutely irreducible on $V$. Similarly if $G \leq S U_{3}(2)$ and $S U_{3}(2)$ acts on $N$, then $\mathrm{O}_{3}\left(S U_{3}(2)\right) \leq C(N)$, whereas $\mathrm{O}_{3}\left(S U_{3}(2)\right)$ is absolutely irreducible on $V$.

If $G=L_{2}(2)$ or $U_{2}(2)$, we calculate to obtain (i) or (ii).

\section{Section 10. Certain normal subgroups of centralizers.}

In this section we assume $G$ is equal to $S L_{n}(q), S U_{n}(q), S p_{n}(q)$, or $\Omega_{n}^{\varepsilon}(q), n \geq 4$, and $q$ a power of 2 . We exclude the cases $L_{4}(2) \cong A_{8}$ and $S p_{4}(2) \cong S_{6}$. If $G$ is orthogonal we take $n \geq 8$.

Let $t$ be an involution of $G$ of rank $\ell, C=C_{G}(t)$ and $\Delta=t^{G} \cap C$. Let $u$ be an involution in $O_{2}(C)$ such that either $[u, \Delta]=1$ or $\langle u, t\rangle \unlhd C$. We wish to determine all possibilities for $u$. This is done in 10.6-10.8.

Let $z=z_{\ell} \cap G, T=T_{\ell} \cap G$, and $\bar{C}=C / T$. Set $K=O^{2^{\prime}}(C)$, except if $t$ is of type $c_{\ell}$ we take only those matrices in (7.11) (1) having $\alpha=0$ and $x=0$. Then $K / T=L_{1} / T \times L_{2} / T$, where the factors are unimodular, unitary, symplectic, or orthogonal groups.

Notice $\overline{K \cap L_{i}}$ is quasisimple unless $\bar{L}_{i}$ is $O_{m}^{s}(q), L_{2}(2), U_{2}(2), S U_{3}(2)$, or $S p_{4}(2)$. Therefore

(10.1) $\overline{K \cap L_{i}}$ is generated by any one of its classes of involutions unless $\bar{L}_{i} \cong S p_{4}(2)$ and $\bar{L}_{i}$ is generated by involutions of type $a_{2}$ or $b_{1}$, or $\bar{L}_{i} \cong O_{m}^{s}(q)$ and $\bar{L}_{i}$ is generated by transvections.

(10.2) $\bar{K}=\overline{\langle K \cap \Delta\rangle}$.

Proof. Let $D=\langle K \cap \Delta\rangle$. By 10.1 it suffices to exhibit conjugates $r$ and $s$ of $t$ in $K$ such that $1 \neq \bar{r} \in \overline{K \cap L_{1}}$ and $\bar{s}$ projects nontrivially on $\overline{K \cap L_{2}}$, with the projections of $r$ and $s$ in a suitable class if $\overline{K \cap L_{i}}$ is 
$S p_{4}(2)$ or $O_{m}^{s}(q)$. The case where $G$ is unimodular or unitary is left to the reader. Let $G$ be symplectic or orthogonal. $r$ and $s$ are exhibited below for $a_{\ell}, b_{\ell}$, and $c_{\ell}$. In each case $W$ is a suitable involution of maximal possible rank. $m=n-2 \ell . \quad J$ is the $2 \times 2$ matrix all of whose entries are 1. If $n=2 \ell$ and $t=a_{\ell}$, then (10.2) follows from (8.12)(2).

$$
a_{\ell}\left(\begin{array}{ccc}
W & & \\
& I_{m} & \\
& & W
\end{array}\right),\left(\begin{array}{llllll}
I_{2} & & & & & \\
& W & & & & \\
& & E_{2} & & & \\
& & & I_{m-2} & & \\
J & & & & I_{2} & \\
& & & & & W
\end{array}\right)
$$
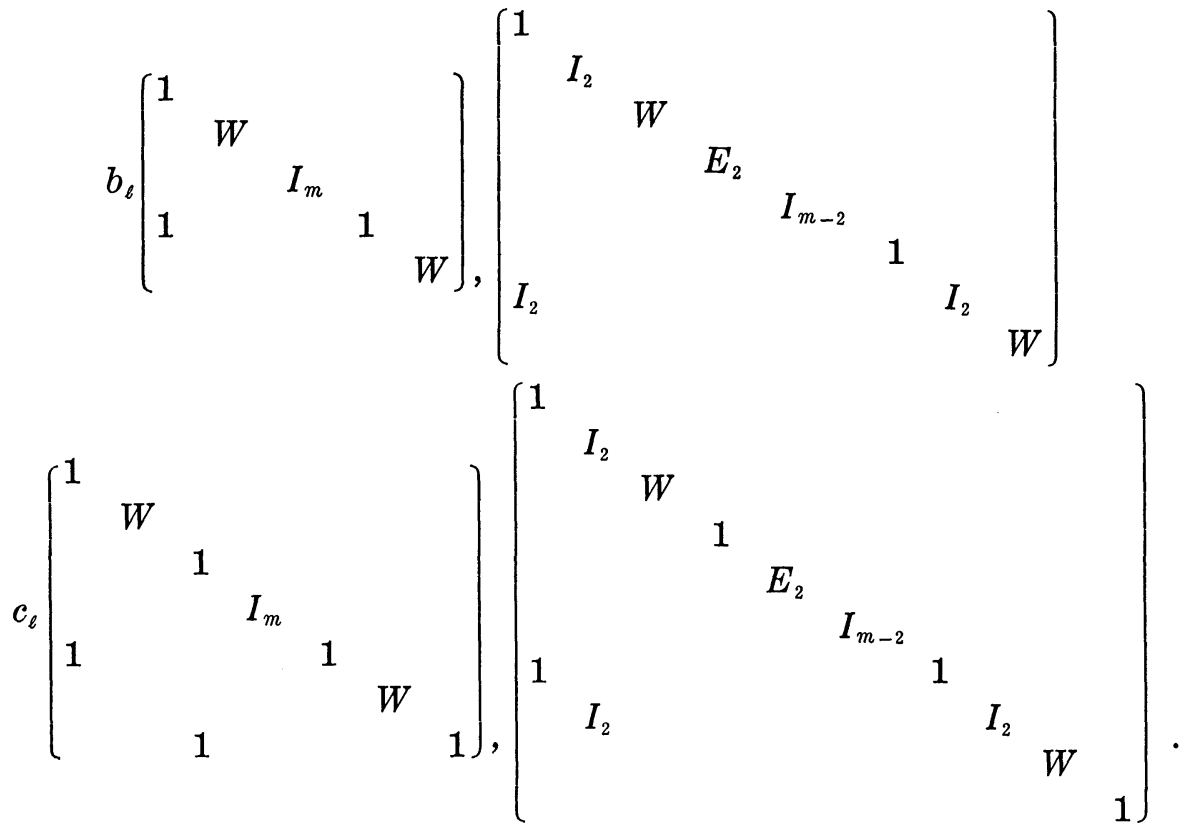

Let $g \in C$ and set $X=X(g), Y=Y(g)$, etc. Recall the definition of $u$.

(10.3) If $u \notin T$, then $t=c_{2}$ and

$$
X(u)=\left[\begin{array}{ll}
1 & \\
d & 1
\end{array}\right]
$$

with $d \neq 0$. If $G$ is symplectic then $q=2$.

Proof. $\quad T=O_{2}(C)$ unless $t=c_{\ell}$, so take $t=c_{\ell}$. Then by (7.11) (1) 


$$
X=\left[\begin{array}{ccc}
1 & & \\
W E \alpha^{*} & W & \\
x & \alpha & 1
\end{array}\right]
$$

and if $g=u$ then $W=I$. Let $U$ be the set of elements in $O_{2}(C)$ with $\alpha=0$. Then by 4.5 the action of $g$ on $O_{2}(C) / U$ is determined by $\alpha\left(x^{g}\right)$ $=\alpha(x) W . \quad \ell$ is even so by 10.2 and 9.1 we conclude $u \in U$. So

$$
X(u)=\left[\begin{array}{lll}
1 & & \\
& I & \\
d & & 1
\end{array}\right]
$$

Suppose $\ell>2$. By 7.11 and 7.13, if $g \in Z$ then

$$
Q=\left[\begin{array}{lll}
r & \rho & z \\
\mu & L & \eta \\
y & \xi & s
\end{array}\right] \quad Q\left(u^{g}\right)=\left[\begin{array}{ccc}
r+z d & \rho & z \\
\mu+d \eta & L & \eta \\
y+(r+s+z d) d & \xi+d \rho & s+d z
\end{array}\right]
$$

so that $[g, u]=1$ exactly when $z=\eta=\rho=0=r+s$. However if $\ell \neq 2$ then by 8.9 the element $g \in Z$ with

$$
Q=\left(\begin{array}{lllll}
1 & 1 & 1 & & \\
& & 1 & & 1 \\
& 1 & & & 1 \\
& & & I_{\ell-4} & \\
& 1 & 1 & & 1
\end{array}\right)
$$

is in $\Delta$, so that $[u, \Delta] \neq 1$ and $\langle u, t\rangle \unlhd C$. Also by 7.12 and $8.9,\left|Z: C_{Z}(u)\right|$ $>2$ only if $\ell=2, G$ is symplectic, and $q>2$.

(10.4) $\quad R(u)=P(u)=0$.

Proof. Let $g \in K$. By 4.5 and 10.3 the action of $g$ on $u Z$ is determined by $P\left(u^{g}\right)=Y^{-1} P(u) X$ and $R\left(u^{g}\right)=X^{-1} R(u) Y$. As $n>3$, either $n-2 \ell$ or $\ell$ is greater than 1 , and if $t=a_{\ell}$ then $\ell>1$. Hence by 9.1 and 10.2, $P(u)=R(u)=0$ if $G$ is unimodular or unitary, or if $t=a_{\ell}$.

Suppose $t=b_{\ell}$. Then by 7.10

$$
R(u)=\left[\begin{array}{l}
\alpha \\
A
\end{array}\right] \quad P(u)=E R^{*} F \quad X^{-1}=\left[\begin{array}{ll}
1 & \\
& W
\end{array}\right]
$$

and then

$$
X^{-1} R(u) Y=\left[\begin{array}{c}
\alpha Y \\
W A Y
\end{array}\right]
$$


As $t=b_{\ell}, u \in T$, so $R(u)=X^{-1} R(u) Y$ for every $g \in C$. By 10.2, $\bar{K}=$ $\langle\overline{K \cap \Delta}\rangle$. Further $n-2 \ell$ is even so if $n \neq 2 l$ then $n-2 \ell>1$. Thus by $9.1, R(u)=0$.

A similar argument works if $t=c_{\ell}$ and $u \in T$. So assume $u \notin T$. Then by $10.3, t=c_{2}$. If $G$ is symplectic then by $10.3 q=2$, so $n>4$, and $\bar{L}_{2} \cong S p_{n-4}(q)$. If $G$ is orthogonal then $n>6$, so $n-2 \ell=n-4 \geq 4$ and $\bar{L}_{2} \cong S p_{n-4}(q)$. Hence by $9.1, R(u)=0$.

(10.5) $u \in Z$.

Proof. Assume not. By 10.4, $R(u)=P(u)=0$, so $u \notin T$. By 10.3, $t=c_{2}$ and

$$
X(u)=\left[\begin{array}{ll}
1 & \\
d & 1
\end{array}\right]
$$

As $R(u)=0$, the image of $g Z$ under $u$ is determined by $R\left(u^{g}\right)=X(u) R$. Further

$$
R=\left[\begin{array}{l}
\beta \\
\gamma
\end{array}\right] \quad X(u) R=\left[\begin{array}{l}
\beta \\
d \beta+\gamma
\end{array}\right]
$$

so $u$ centralizes $g Z$ exactly when $\beta=0$. However as $g$ ranges over $C$, $\beta$ ranges over all possible row vectors of length $n-4$, so unless $n=4$, $\left|u^{C}\right|>2$. Also if $n>4$ then

$$
g=\left(\begin{array}{lllllll}
1 & & & & & \\
& 1 & & & & \\
& & E_{2} & & & \\
& & & I & & \\
0 & 1 & & & 1 & \\
0 & 1 & & & & 1
\end{array}\right)
$$

is in $\Delta$ by 8.9 , while by $4.5, Q\left(u^{g}\right) \neq Q(u)$.

So $n=4$ and hence $G$ is symplectic. Now by $10.3, G=S p_{4}(2)$, against our hypothesis.

(10.6) Let $t=j_{\ell}$ or $a_{\ell}$. Then

(1) if $[u, \Delta]=1$ then $u \in J_{\ell}$, and

(2) if $u^{c}=\{u, u t\}$ then either

(i) $G=S L_{n}(2)$ or $S p_{n}(2), \ell=2$, and $Q(u)$ is

$$
\left[\begin{array}{ll}
1 & 1 \\
1 & 0
\end{array}\right] \text { or }\left[\begin{array}{ll}
0 & 1 \\
1 & 1
\end{array}\right]
$$


(ii) $\quad G=S U_{n}(2), \ell=2$, and $Q(u)$ is

$$
\left[\begin{array}{ll}
1 & 0 \\
0 & 0
\end{array}\right] \text { or }\left[\begin{array}{ll}
0 & 0 \\
0 & 1
\end{array}\right]
$$

Proof. $u^{g}$ is determined by $Q\left(u^{g}\right)=Q(u)^{X}$. Thus the result follows from 9.2 and 10.2 .

(10.7) Let $t=b_{\ell}$. Then

(1) if $[u, \Delta]=1$, then

$$
Q(u)=\left[\begin{array}{ll}
b & \\
& a I
\end{array}\right]
$$

(2) $u^{C} \neq\{u, u t\}$.

Proof. By 7.10,

$$
Q(u)=\left[\begin{array}{ll}
b & \beta \\
\gamma & L
\end{array}\right] \quad X=\left[\begin{array}{ll}
1 & \\
& W
\end{array}\right] \quad Q\left(u^{g}\right)=\left[\begin{array}{cc}
b & \beta^{W} \\
W^{-1} \gamma & L^{W}
\end{array}\right]
$$

where $\beta$ and $\gamma$ are respectively row and column vectors of even length $\ell-1$. So by 9.2 and 10.2, $\gamma=\beta=0$ and $L=a I$ if $[u, \Delta]=1$. Also if $u^{C}=\{u, u t\}$ then

$$
Q(u)+Q\left(u^{g}\right)=\left[\begin{array}{ll}
0 & * \\
* & *
\end{array}\right] \neq I .
$$

(10.8) Let $t=c_{\ell}$. Then

(1) if $[u, \Delta]=1$ then

$$
Q(u)=\left[\begin{array}{lll}
r & & \\
& r I & \\
y & & r
\end{array}\right]
$$

and if $G$ is orthogonal $y=r(r+1)$.

(2) If $u^{C}=\{u, u t\}$ then $G=S p_{n}(2), \ell=2$, and $Q(u)$ is one of

$$
\left[\begin{array}{ll}
0 & 1 \\
1 & 1
\end{array}\right],\left[\begin{array}{ll}
1 & 1 \\
1 & 0
\end{array}\right],\left[\begin{array}{ll}
1 & 1 \\
0 & 0
\end{array}\right],\left[\begin{array}{ll}
0 & 1 \\
0 & 1
\end{array}\right]
$$

Proof. By 7.11

$$
Q(u)=\left[\begin{array}{lll}
r & \rho & z \\
\mu & L & \eta \\
y & \xi & s
\end{array}\right] \quad X=\left[\begin{array}{ccc}
1 & & \\
W E \alpha^{*} & W & \\
x & \alpha & 1
\end{array}\right] .
$$


Choosing $\alpha=x=0$ we find

$$
Q\left(u^{g}\right)=\left[\begin{array}{ccc}
r & \rho^{W} & z \\
W^{-1} \mu & L^{W} & W^{-1} \eta \\
y & \xi W & s
\end{array}\right]
$$

so by 9.1 and 10.2, $\mu=\rho=\eta=\xi=0$ and $L=a I$ is scalar. Choosing $W=I$, it follows from 7.13 that

$$
Q\left(u^{g}\right)=\left[\begin{array}{ccc}
r+z x & z \alpha & z \\
(r+z x+a) E \alpha^{*} & L+z E \alpha^{*} \alpha & z E \alpha^{*} \\
y+(r+s+z x) x & \alpha(a+s+x z) & x z+s
\end{array}\right] .
$$

So as $C_{C}(\Delta) \unlhd C$ we conclude either $\ell=2$ or $z=0$ and $a=r=s$. Further if $G$ is orthogonal then by $8.9, y=r(r+1)$.

So take $\ell=2$. Then if $G$ is orthogonal, 8.11 implies $r=s, z=0$, and $y=r(r+1)$. So take $G$ symplectic. Then for $x \neq 1$

$$
g=\left(\begin{array}{lllll}
1 & & & & \\
x & 1 & & & \\
& & I & & \\
& & & 1 & \\
1 & & & x & 1
\end{array}\right)
$$

is in $\Delta$, so if $[u, \Delta]=1$ then $z=0$ and $r=s$. Also if $[u, \Delta] \neq 1$ then as $Q(u)=Q\left(u^{g}\right)+I$ for each such $g, q=2$ and $Q(u)$ is as claimed.

\section{Section 11. Alpha and beta groups.}

In this section we continue the hypothesis stated in the first paragraph of Section 10. In addition we take $q>2$.

A primary involution is defined to be a transvection in the unimodular, unitary, or symplectic group, and an involution of type $a_{2}$ in the orthogonal group.

Given an involution $t \in G$ define $\alpha(t)$ to be the set of elements $g$ in $G$ such that $g+I=a(t+I)$ for some scalar $\alpha . \alpha(t)$ is the $\alpha$-group of $G$ containing $t$. In addition if $t$ is of type $c_{\ell}$ or of type $b_{\ell}$ let $\beta(t)$ be the group generated by all involutions $s$ in $G$ such that $V(s)=V(t)=U$ and

$$
\begin{gathered}
\left.(I+s)\right|_{U}=\left.a(I+t)\right|_{U} \\
I+s \equiv a(I+t) \bmod U^{\perp}
\end{gathered}
$$


for some scalar $a . \quad \beta(t)$ is the $\beta$-group of $G$ containing $t$.

In this section we investigate the $\alpha$ and $\beta$-groups and the primary involutions. The next two lemmas relate the $\alpha$ and $\beta$-groups to the results in Section 10. For it is easy to check that

(11.1) Let $t$ be in Suzuki form. Then $\alpha(t)=J_{\ell} \cap G$.

(11.2) Let $t=c_{\ell} \in \Omega^{\mathrm{s}}$. Then $z \in \beta(t)$ exactly when $z \in Z$ with

$$
Q(z)=\left[\begin{array}{ccc}
r & & \\
r(r+1) & & r I
\end{array}\right] .
$$

Therefore the results in Section 10 imply $\alpha(t)$, or $\beta(t)$ if $t$ is of type $b_{\ell}$ or $c_{\ell}$, is the center of the centralizer of $t$.

(11.3) Let $t$ be an involution of rank $\ell>1$ in $S L$ or $S U$. Then there exists $s \in t^{G} \cap C$ such that $\alpha(t) \alpha(s)$ contains one $\alpha$-group of rank 1 , one of rank $\ell-1$, and $q-1 \alpha$-groups of rank $\ell$.

Proof. Take $t$ in Suzuki form and for scalars $a$ and $b$ define $g(a, b) \in C(t)$ by

$$
g(a, b)=\left(\begin{array}{lllll} 
& & I_{\ell} & & \\
& & & I_{n-2 \ell} & \\
a & & & & \\
& b & & & \\
& & I_{\ell-2} & & I_{\ell}
\end{array}\right) .
$$

Let $s=g(1, c), 0 \neq c \neq 1$. Then

$$
\alpha(g(a, b))=\{g(d a, d b)\}
$$

$g(1,0)$ is of rank $1, g(0,1)$ is of rank $\ell-1$, and the remaining $\alpha$-groups have rank $\ell$.

The next two lemmas are proved in the same manner.

(11.4) Let $t=a_{\ell}, \ell>2$. Then there exists $s \in t^{G} \cap C$ such that $\alpha(t) \alpha(s)$ contains one $\alpha$-group of type $a_{2}$, one of type $a_{\ell-2}$, and $q-1$ of type $a_{\ell}$.

(11.5) Let $t=c_{\ell}, \ell>2$, or $t=b_{\ell}, \ell>1$. Then there exists $s \in t^{G} \cap C$ such that $\beta(t) \beta(s)$ contains one $\alpha$-group of type $a_{\ell-2}$, or $a_{\ell-1}$, one $\beta$-group of type $c_{2}$, or $b_{1}$, and $q-1 \beta$-groups of type $c_{\ell}$, or $b_{\ell}$, respectively.

(11.6) Let $t=a_{2}$ or $t=b_{1}$. Then there exists $s \in t^{G} \cap C$ such that $\alpha(t)$ 
and $\alpha(s)$ are the unique $\alpha$-groups of type $t$ in $\alpha(t) \alpha(s)$ and the remaining $q-1 \alpha$ or $\beta$-groups are of type $c_{2}$.

Proof. This can be proved as above for the symplectic groups. In the case of the orthogonal groups $t=a_{2}$ and it is easier to recall $\Omega_{6}^{+}(q) \cong L_{4}(q), \Omega_{6}^{-}(q) \cong U_{4}(q)$, and argue directly.

(11.7) Let $t$ be a primary involution. Then $C_{G}(t)^{\infty}=T L$.

Proof. Assume $t$ is a transvection in Suzuki form. For $x \in T$ and $g \in L, R(x)$ is a row vector of length $m=n-2$ and $R\left(x^{g}\right)=R(x) Y(g)$. Thus $L$ acts on the space $U$ of row vectors $R(x)$ in its natural representation. $L$ is irreducible on $U$. Hence $U=[U, L]$, so $T / Z=[T / Z, L]$ and then $T=[T, L] Z$. So as $L$ is quasisimple it suffices to show $Z \leq$ $[T, L]$.

If $G$ is unimodular or unitary then an easy calculation shows $Z=$ $\Phi(T)$, so $Z \leq[T, L]$. If $G$ is symplectic $Z \leq[T, L]$ follows from $\S 3$ of [7].

Next assume $G$ is orthogonal and $t=a_{2}$. Arguing as above $T / Z=$ $[T / Z, L]$. As $\Omega_{6}^{+}(q) \cong L_{4}(q)$ and $\Omega_{6}^{-}(q) \cong U_{4}(q)$, with $a_{2}$ corresponding to a transvection under the respective isomorphism, $Z \leq\left[T_{0}, L_{0}\right]$ where $T_{0}=$ $T \cap G_{0}, L_{0}=L \cap T_{0}$, and $Z \leq G_{0} \leq G$ with $G_{0} \cong \Omega_{6}^{\epsilon}(q)$.

(11.8) Let $t_{i} \in G$ be transvections, $\left\langle v_{i}\right\rangle=\left[t_{i}, V\right], V_{i}=C_{V}\left(t_{i}\right)$, and $W=$ $\left\langle\alpha\left(t_{1}\right), \alpha\left(t_{2}\right)\right\rangle$. Then

(1) $\left[t_{1}, t_{2}\right]=1$ if and only if $v_{i} \in V_{j}$ for $i \neq j$.

(2) $W \cong L_{2}(q)$ if and only if $v_{i} \notin V_{j}$ for $i \neq j$.

(3) $G$ is transitive on subgroups isomorphic to $L_{2}(q)$ containing the $\alpha$ group of a transvection.

(4) If $W \cong L_{2}(q)$ then $C_{G}(W)$ is isomorphic to $G L_{n-2}(q), G U_{n-2}(q)$, or $S p_{n-2}(q)$ for $G$ unimodular, unitary, or symplectic, respectively. Further $W C_{G}(W)$ is not centralized by an involutory automorphism of $G$. Unless $G=L_{4}(q)$ or $U_{4}(q)$, the same holds for $W E\left(C_{G}(W)\right)$.

Proof. (1), (2), and the first part of (4) follow by calculation choosing suitable bases for $V$. By [21] the automorphism group of $G$ is known, and the remainder of (4) follows by inspection.

A flag is an incident point hyperplane pair. The unimodular group is transitive on pairs of flags $\left(\left(v_{1}, V_{1}\right),\left(v_{2}, V_{2}\right)\right)$ with $v_{i} \notin V_{j}, i \neq j$. If $G$ 
is unitary or symplectic then $V_{i}=v_{1}^{\perp}$ and $G$ is transitive on pairs $\left(v_{1}, v_{2}\right)$ with $v_{1} \notin V_{2}$. Hence (2) implies (3).

(11.9) ${ }^{\circ}$ Let $G=\Omega_{n}^{\varepsilon}(q), t=a_{2}$ and $s \in t^{G}$. Set $U=[t, V]$ and $W=\langle\alpha(t), \alpha(s)\rangle$. Then

(1) $Z=\alpha(t)$.

(2) $s \in \alpha(t)$ if and only if $U=[s, V]$.

(3) $[s, t]=1$ if and only if $s$ stabilizes $U$.

(4) $\left[t, t^{s}\right]=1$ if $U^{\perp} \cap U^{s} \neq 0$.

(5) $W$ is isomorphic to a Sylow 2-group of $L_{3}(q)$ if and only if $0 \neq$ $U^{\perp} \cap U^{s} \neq U$.

(6) $W \cong L_{2}(q)$ if and only if $0=U^{\perp} \cap U^{s}$ if and only if $U+U^{s} \cong D^{2}$.

(7) $G$ is transitive on subgroups isomorphic to $L_{2}(q)$ containing the $\alpha$ group of a primary involution.

(8) If $W \cong L_{2}(q)$ then $C_{G}(W) \cong L_{2}(q) \times \Omega_{n-4}^{*}(q)$, and $W C_{G}(W)$ is not centralized by an involutory automorphism of $G$.

Proof. (1) follows from an easy calculation. Assume $U=[s, V]$. Then $C_{V}(s)=U^{\perp}=C_{V}(t)$, so $s \in Z$, and (1) implies (2). (3) follows from (2).

Assume $0 \neq u^{s} \in U^{\perp} \cap U^{s}$. Let $v \in U-\langle u\rangle . \quad 0=\left(u^{s}, U\right)=\left(u, U^{s}\right)$. $s \in t^{G}$, so $V(s)=V$. Hence $\left(v, v^{s}\right)=0$. So $U^{s}=\left\langle u^{s}, v^{s}\right\rangle \leq v^{\perp}$. Hence $U^{s} \leq U^{\perp}$, so $\left[U^{s}, t\right]=0$, and then $\left[t^{s}, t\right]=1$. This is $(4)$.

Assume $0 \neq U^{\perp} \cap U^{s} \neq U$. Let $A=[s, V] . \quad U+A$ is contained in a nondegenerate 6-dimensional space $B$. As $U+A \leq B, B$ is $W$-invariant so $W \leq \Omega(B)$. $\quad \Omega(B) \cong L_{4}(q)$ or $U_{4}(q)$ with $t$ corresponding to a transvection under the isomorphism, so $W$ is isomorphic to $L_{2}(q)$ or a Sylow 2-group of $L_{3}(q)$. As $\left[t, t^{s}\right]=1$, it is the latter. This proves half of (5).

If $U^{s} \cap U^{\perp}=0$ then $[V, s] \leq Y=U+U^{s}$ is nondegenerate and $W$-invariant, so $W \leq \Omega(Y)$. Hence $Y \cong D^{2}, \Omega(Y) \cong L_{2}(q) \times L_{2}(q)$ and $W$ is a factor of $\Omega(Y)$. Further $C_{G}(W)=(C(W) \cap \Omega(Y)) \times \Omega\left(Y^{\perp}\right) \cong L_{2}(q) \times \Omega_{n-4}^{s}(q)$. This completes (5) and shows that (6)-(8) follow from (3) and (5).

(11.10) Let $t$ be a transvection and $s$ an involution in $G$ such that $C_{G}(t)^{\infty} \cong C_{G}(s)^{\infty}$. Then either $s$ is a transvection or $G=S p_{4}(q)$, $s$ is of type $a_{2}$, and $t^{G}$ is fused to $s^{G}$ by a graph automorphism.

Proof. Let $X=C(t)^{\infty}$ and $Y=C(s)^{\infty} . \quad X / O_{2}(X) \cong G_{n-2}(q)$ where $G=S L, S U$, or $S p$. But the factors of $Y / O_{2}(Y)$ are $G_{n-2 \ell}(q)$ and $G_{k}(q)$ 
where $\ell$ is the rank of $s$ and $k=\ell, \ell-1$ or $\ell-2$. We may take $\ell>1$, so $n-2 \leq \ell \leq n / 2$. Thus $n=4$ and $k=\ell=2$. However $O_{2}\left(C\left(j_{2}\right)\right)$ is abelian while $O_{2}\left(C\left(j_{1}\right)\right)$ is not. Hence $G=S p_{4}(q)$ and $s$ is of type $a_{2}$

(11.11) Let $t=\alpha_{2} \in \Omega_{n}^{s}(q)$ and $s$ an involution in $G$ with $C(s)^{\infty} \cong C(t)^{\infty}$, then $s$ is primary.

Proof. $C^{\infty} / O_{2}(C) \cong L_{2}(q) \times \Omega_{n-4}^{\varepsilon}(q)$. Further unless $n=8$ and $\varepsilon=+$, $\Omega_{n-4}^{\varepsilon}(q)$ is simple and only primary involutions have a factor of this kind. If $n=8$ and $\varepsilon=+$ then $\Omega_{n-4}^{\varepsilon}(q) \cong L_{2}(q) \times L_{2}(q)$ and only primary involutions have 3 factors.

(11.12) Let $t=j_{1}$ or $b_{1}$. Let $h$ be an involution in $C-T$ and $g \in C$ with

$$
h=\left[\begin{array}{lll}
1 & & \\
A & H & \\
B & C & 1
\end{array}\right] \quad g=\left[\begin{array}{lll}
X & & \\
P & Y & \\
Q & R & X
\end{array}\right]
$$

Then

(1) $H^{2}=I, H A=A, C H=C$ and $C A=0$.

(2) If $[Y, H]=1$ then

$$
h^{g}=\left[\begin{array}{ccc}
1 & & H \\
Y^{-1}(A X+P+H P) & 1
\end{array}\right] .
$$

(3) Assume $A=\left(a_{1}, \cdots, a_{m}\right)^{*}, C=\left(c_{1}, \cdots, c_{m}\right)$, and

$$
H=\left[\begin{array}{ll}
E_{2} & \\
& I
\end{array}\right] \text {. }
$$

Then $a_{1}=a_{2}$ and $c_{1}=c_{2}$.

Proof. Calculation using 4.4 and 4.5.

(11.13) Assume the hypothesis of 11.12 with $h$ as in 11.12 .1 and

$$
e=\left[\begin{array}{lll}
1 & & \\
& H & \\
& & 1
\end{array}\right]
$$

Assume $C_{T}(e) \cong C_{T}(h)$ and $C^{\infty} \cap C(e) \cong C^{\infty} \cap C(h)$. Then $h \in e Z$.

Proof. Let $g \in C_{T}(h)$. Then by 11.12, $P=H P$ and $R=R H$, so that 
$C_{T}(h) \leq C_{T}(e)$. Thus as $C_{T}(e) \cong C_{T}(h)$, even $C_{T}(e)=C_{T}(h)$. Hence 11.12 implies $R A=C P$ whenever $g \in C_{T}(e)$. This implies $a_{i}=c_{i}=0$ for $i>2$. By 11.12, $a_{1}=a_{2}$ and $c_{1}=c_{2}$. Next as $C^{\infty} \cap C(e) \cong C^{\infty} \cap C(h), h$ centralizes an element $g$ with

$$
Y=\left[\begin{array}{lll} 
& b^{-1} & \\
b & & \\
& & I
\end{array}\right] .
$$

By 11.12, $C=C Y+R H+R$, which implies $c_{1}=0$. Similarly $a_{1}=0$. Therefore $h \in e Z$.

(11.14) Let $t$ be a transvection and $\beta$ an automorphism of $C^{\infty}$. Let

$$
e=\left(\begin{array}{llll}
1 & & & \\
& E_{2} & & \\
& & I & \\
& & & 1
\end{array}\right) .
$$

Then $e^{\beta}$ is a transvection and $\alpha(e)^{\beta}=\alpha\left(e^{\beta}\right)$.

Proof. Let $h=e^{\beta}$. By 11.7, $C^{\infty}=T L . \quad C_{C^{\infty / T}}(e) \cong C_{C^{\infty} / T}(h)$, so by 11.10 either $h T$ is a transvection in $C / T$ or $C / T \cong S p_{4}(q)$ and $h T$ is of type $a_{2}$. However in the latter case $q^{3}=\left|C_{T}(e)\right|$ while $q^{2}=\left|C_{T}(h)\right|$. Hence conjugating if necessary in $C^{\infty}$ we may take $h \in e T$.

Then by 11.13, $h \in e Z$. Further the involutions in $\alpha(e) Z=X$ are precisely those with the same centralizer as $e$ (or $h$ ) and thus $\beta$ acts on $X$. But $\alpha(e)=[X, N(X)]$, so $\alpha(e)^{\beta}=\alpha(e)$. Hence $h \in \alpha(e)$, so $h$ is a transvection and $\alpha(e)^{\beta}=\alpha(e)=\alpha(h)$.

(11.15) Let $t=a_{2} \in \Omega_{n}^{2}(q)$. Let $\beta$ be an automorphism of $C^{\infty}$ and

$$
e=\left[\begin{array}{lll}
E_{2} & & \\
& I & \\
& & E_{2}
\end{array}\right] \text {. }
$$

Then $e^{\beta}$ is of type $a_{2}$ and $\alpha(e)^{\beta}=\alpha\left(e^{\beta}\right)$.

Proof. The proof is similar to that of 11.14 and is omitted.

\section{Section 12. Involutions in Exceptional Chevalley groups of characteristic 2.}

Recall the notation for groups with a $(B, N)$-pair established in Section 3. Let $G=G(q)$ be a Chevalley group with $q$ even, and $G \neq F_{4}(q)$, $P S p(n, q) n \geq 4$. Let $G$ have root system $\Delta$. For $G \neq P S U(n, q) n$ odd, 
${ }^{2} F_{4}(q), S z(q),{ }^{2} G_{2}(q)$ let $r$ be the root of highest height in $\Delta$ and $U_{r}$ the corresponding root subgroup. In the remaining cases let $U_{r}$ be the root subgroup of order $q^{3}, q^{2}, q^{2}, q^{3}$ respectively, such that $\Omega_{1}\left(U_{r}\right)=Z(U)$. For $s \in \Delta$ write $Z_{s}=\Omega_{1}\left(U_{s}\right)$. In all cases $Z_{r} \leq Z(U)$ and, unless $G=P S L(n, q)$, $P=N_{G}\left(Z_{r}\right)$ is a maximal parabolic subgroup of $G$.

The structure of $P$ is described in [7]. In particular $P=Q L H$ where $Q=O_{2}(P), L$ is a Chevalley group (or a direct product of Chevalley groups in case $G$ is an orthogonal group of dimension $\geq 8$ ), $Q L \unlhd P$ and $Q L \leq C\left(Z_{r}\right) \unlhd P$.

(12.1) Two conjugates of $Z_{r}$ generate a 2-group or a conjugate of $\left\langle Z_{r}, Z_{-r}\right\rangle \cong S L(2, q)$.

Proof. Let $\Omega=\left\{Z_{r}^{g}: g \in G\right\}$. Then from 2.8 and 4.2 of [7] we see that $\left(1_{P}^{G}, 1_{P}^{G}\right)=m$ is the number of orbits of $P$ on $\Omega$ and

$m=2$ if $G$ has rank 1

$m=3$ if $G$ is unitary or symplectic of rank $\geq 2$.

$m=4$ if $G=G_{2}(q)$ or ${ }^{3} D_{4}(q)$

$m=5$ if $G$ is an exceptional group of rank $\geq 3$ or if $G \cong{ }^{2} F_{4}(q)$.

$m=6$ if $G \cong \operatorname{PSO}^{-}(2 n, q), n \geq 4, \operatorname{PSO}^{+}(n, q) n>4$, or $\operatorname{PSL}(n, q) n \geq 3$.

$m=1$ if $G \cong \operatorname{PSO}^{+}(8, q)$ (there is an error in [7] for this case).

We will do the case $m=5$, the other cases being quite similar. Consider the following subsets of $\Omega$.

$$
\begin{aligned}
& \Omega_{1}=\left\{Z_{r}\right\}, \quad \Omega_{2}=\left\{Z_{r}^{g}: Z_{r} \neq Z_{r}^{g} \leq Q\right\}, \quad \Omega_{3}=\left\{Z_{r}^{g}: Z_{r}^{g} \leq P-Q\right\}, \\
& \Omega_{4}=\left\{Z_{r}^{g}:\left\langle Z_{r}, Z_{r}^{g}\right\rangle \text { is isomorphic to a Sylow 2-subgroup of } L_{3}(q)\right\}, \\
& \Omega_{5}=\left\{Z_{r}^{g}:\left\langle Z_{r}, Z_{r}^{g}\right\rangle \cong\left\langle Z_{r}, Z_{-r}\right\rangle \cong S L(2, q)\right\} .
\end{aligned}
$$

We first note that each of these subsets is $P$-invariant and it is easy to verify that $\Omega_{i} \neq \emptyset$ for $i=1, \cdots, 5$. This requires information concerning the root system $\Delta$ and commutator relations, but is elementary. Consequently these are the orbits of $P$ in $\Omega$. If $Z_{r}^{g} \in \Omega_{1} \cup \Omega_{2} \cup \Omega_{3} \cup \Omega_{4}$ then $\left\langle Z_{r}, Z_{r}^{g}\right\rangle$ is a 2-group. The result follows.

We remark that for $G=P S O^{ \pm}(2 n, q) n \geq 4\left(G \neq P S O^{+}(8, q)\right)$, we have $L \cong L_{1} \times L_{2}$ with conjugates of $Z_{r}$ in each of $L_{1}, L_{2}$. If $G=P S O^{+}(8, q)$, then $L=L_{1} \times L_{2} \times L_{3}$ with each $L_{i} \cong L_{2}(q)$. This accounts for the extra orbit. In $P S L(n, q) Q=Q_{1} Q_{n}$ with $Q_{1} \triangleleft P, Q_{n} \triangleleft P$. This also gives an extra orbit.

(12.2) Let $t$ be an involution in $G$. Then there is a conjugate $Z_{r}^{g}$ of $Z_{r}$ 
such that $\left\langle Z_{r}^{g}, Z_{r}^{g t}\right\rangle \sim\left\langle Z_{r}, Z_{-r}\right\rangle$.

Proof. If $G$ has rank 1 this is obvious. For $G$ of rank 2 the classes of involutions are known (see Section 18) and we check this directly (keeping in mind $G \not S p(4, q)$ ). So we may assume $G$ is of rank $n \geq 3$ and we proceed by induction on $n$. Since $n \geq 3 L$ contains a conjugate of $Z_{r}$. We first conjugate, if necessary, replacing $t$ by an element of $U$. Suppose $t \notin Q$. Then considering the image of $t$ in $L Q / Q=\bar{L}$ we inductively see that there is a conjugate $Z_{r}^{g}$ of $Z_{r}$ such that $\left\langle Z_{r}^{g}, Z_{r}^{g t}\right\rangle$ is not a 2-group. So (12.1) yields the result. Consequently we may assume that $t \in Q$.

If $t \in Z_{r}=Q^{\prime}$, then we obtain the result by considering $t \in\left\langle U_{ \pm r}\right\rangle \cong$ $S L(2, q)$. So assume that $t \in Q-Z_{r}$ and consider the image $\bar{t}$ of $t$ in $\bar{Q}=Q / Z_{r}$. Write $Q=\prod_{i=1}^{k} U_{\beta_{i}}$ when the product is over a certain set of roots in $\Delta^{+}$such that $\beta_{i}=r$ for some $i$ and such that $\bar{Q}=\bar{U}_{\beta_{1}}$ $\times \cdots \times \bar{U}_{\beta_{n}}$. Also $P=\left\langle B, s_{1}, \cdots, s_{j-1}, s_{j+1}, \cdots, s_{n}\right\rangle$ for some $j$ or $G \cong$ $\operatorname{PSL}(n+1, q)$ and $P=\left\langle B, s_{2}, \cdots, s_{n-1}\right\rangle$. In the latter case let $j=1$. We choose notation so that $\bar{U}_{\beta_{1}}=\bar{U}_{\alpha_{j}}$ and $\bar{U}_{\beta_{2}}=\bar{U}_{\alpha_{j}+\alpha_{i}}$ where $i, j$ are given as follows
i) $G \cong P S O^{ \pm}(\ell, q) \quad \ell$ even, $\ell \geq 8$
$j=2 \quad i=1$
ii) $G$ classical not in (i)
$j=1 \quad i=2$
iii) $G \cong F_{4}(q),{ }^{2} E_{6}(q)$
$j=1 \quad i=2$
iv) $G \cong E_{6}(q)$
$j=2 \quad i=4$
v) $G \cong E_{7}(q)$
$j=1 \quad i=3$
vi) $G \cong E_{8}(q)$
$j=8 \quad i=7$.

Suppose that for some $\ell \bar{t}$ projects non-trivially to $\bar{U}_{\beta \ell}$ and $\beta_{\ell} \sim \alpha_{j}$. Then from (2.4) of [7] and the choice of $i$ we have an element $w \in W$ $\cap L$ such that $\bar{t}^{w}$ projects non-trivially to $\bar{U}_{\beta_{2}}=\bar{U}_{\alpha_{j}+\alpha_{i}}$. Using (3.1) (i) we can find an element $u$ of $U_{-\alpha_{i}} \leq L$ such that $\bar{t}^{w u}$ projects trivially to $\bar{U}_{\beta_{1}}$ and non-trivially to $\bar{U}_{\beta_{2}}$. Then conjugation by $s_{j}$ shows that $t^{w u s j} \in$ $U-Q$, reducing to a previous case.

Finally we have the case where no such $\ell$ exists. This only occurs when all non-trivial factors in $\bar{t}$ correspond to roots of length different from that of $\alpha_{j}$. This can only occur when $G={ }^{2} E_{6}(q), P S U(\ell, q) \quad \ell$ odd, $P_{S O}^{-}(\ell, q)$. In these cases we check the possible roots $\gamma$ with $U_{r} \leq Q$ and argue essentially as above, using (3.1).

$$
O^{2^{\prime}}\left(N_{G}\left(\left\langle Z_{r}, Z_{-r}\right\rangle\right)\right)=L \times\left\langle Z_{r}, Z_{-r}\right\rangle
$$


Proof. Let $g \in N_{G}\left(\left\langle Z_{r}, Z_{-r}\right\rangle\right)$. Then setting $X=\left\langle Z_{r}, Z_{-r}\right\rangle$ there is some $x \in X$ such that $g x \in N\left(Z_{r}\right)=P$. Now $P=Q L H$ and $L H \leq N(X)$. So write $g x=q y$ where $q \in Q, y \in L H$. We then have $q \in Q \cap N(X)$ and checking commutators we see that $\left[Z_{-r}, q\right]$ is a 2-group contained in $U^{w_{0}}$, where $w_{0}$ is the word of greatest length in the generators $\left\{s_{1}, \cdots, s_{n}\right\}$. Consequently $\left[Z_{-r}, q\right] \leq U^{w_{0}} \cap X=Z_{-r}$, and $q \in N\left(Z_{-r}\right)$. Then $q \in P \cap P^{w_{0}}$ $=L H$ and so $q=1$. Consequently $g \in X L H$ and the result follows.

(12.4) Let $t \in G$ be an involution. Then some conjugate of $t$ lies in $L Z_{r}(1)$.

Proof. Since $t$ normalizes $\left\langle Z_{r}^{g}, Z_{r}^{g t}\right\rangle$ this follows from (12.2) and (12.3).

We define roots $r=t_{0}, t_{1}, \cdots, t_{k}$ as follows. Let $L$ be the Levi factor of $N\left(Z_{r}\right)$. Then $L$ is a Chevalley group if $G$ is not an orthogonal group, and $L=X \times Y$ with $Y \cong S L(2, q)$ and $X$ orthogonal if $G$ is orthogonal. $\left(X \cong L_{2}(q) \times L_{2}(q)\right.$ if $\left.G \cong P S O^{+}(8, q)\right)$. In the first case let $t_{1}$ be the root of highest height in the root system $\Delta_{1} \subset \Delta$ of $L$. In the second case let $t_{1}$ be the unique root in $\Delta$ with $U_{t_{1}} \leq Y$ and let $t_{2}$ be the highest root in $\Delta_{1}$. Now continue the selection of $t_{i}$ 's by considering $N_{L}\left(Z_{t_{1}}\right)$ (or $N_{X}\left(Z_{t_{2}}\right)$ for $G$ orthogonal). Then setting $X_{i}=\left\langle Z_{t_{i}}, Z_{-t_{i}}\right\rangle$ we have $X \cong S L(2, q)$.

a) $\left\langle X_{i}: i=0, \cdots, k\right\rangle=X_{0} \times \cdots \times X_{k}$.

b) Each involution in $G$ is conjugate to one in $X_{0} \cdots X_{k}$.

c) If $G=P S L(n, q), P S U(n, q), E_{6}(q),{ }^{2} F_{4}(q)$ or ${ }^{2} E_{6}(q)$, then $N_{W}\left(X_{0}\right.$ $\cdots X_{k}$ ) induces $S_{k+1}$ on $X_{0} \cdots X_{k}$ and each involution in $G$ is conjugate to $y_{i}$ for some $i=0, \cdots, k$, where $y_{i}=Z_{r}(1) Z_{t_{1}}(1)$ $\cdots Z_{t_{i}}(1)$.

Proof. a) follows from the construction of the $U_{t_{i}}$ 's, noting that at each stage $X$ is contained in the Levi factor of $N\left(Z_{i-1}\right)$. Also b) follows easily from (12.4) and construction. It remains to prove c). From b) it suffices to prove that $N_{W}\left(X_{0} \cdots X_{k}\right)$ induces $S_{k+1}$ on $X_{0} \cdots X_{k}$. But this follows using induction and calculating with roots to see that there is an element $w \in W$ stabilizing $\left\{X_{0} \cdots X_{k}\right\}$ and interchanging $X_{0}$ and $X_{1}$.

From (12.5) we obtain just a few possibilities for the conjugacy classes of involutions. It remains to narrow this list still further and to find representatives more convenient for finding the centralizers. We 
use the notation and labeling or roots as in Table 1,2,3,4 of Section 3.

We remark that the following results give representatives for the classes of involutions, although we wait until later sections to prove that no further fusion takes place among the involutions listed. We now allow the case $G=F_{4}(q)$.

(12.6) (Guterman [14]) Each involution in $F_{4}(q), q$ even, is conjugate to one of the following:
i) $t=U_{r}(1)$
ii) $u=U_{s}(1)$
iii) $t u=U_{r}(1) U_{s}(1)$
iv) $v=U_{\alpha}(1) U_{\beta}(1)$.

(12.7) Each involution in ${ }^{2} E_{6}(q), q$ even, is conjugate to one of the following:
i) $t=U_{r}(1)$
ii) $u=U_{s}(1)$
iii) $v=U_{\alpha}(1) U_{\beta}(1)$.

Proof. Here $P=P_{1}=Q_{1} L_{1} H=N\left(Z_{r}\right)$, where $L_{1}=\left\langle U_{ \pm \alpha_{2}}, U_{ \pm \alpha_{3}}, U_{ \pm \alpha_{4}}\right\rangle$ $\cong S U(6, q)$. Then $t_{1}=\alpha_{2}+2 \alpha_{3}+2 \alpha_{4}=r_{17}, t_{2}=\alpha_{2}+2 \alpha_{3}$, and $t_{3}=t_{k}=\alpha_{2}$. Then by (12.5) c) each involution in ${ }^{2} E_{6}(q)$ is conjugate to one of

$$
U_{r}(1), \quad U_{r}(1) U_{r_{17}}(1), \quad U_{r}(1) U_{r_{17}}(1) U_{\alpha_{2}+2 \alpha_{3}}(1), \quad U_{r}(1) U_{r_{17}}(1) U_{\alpha_{2}+2 \alpha_{3}}(1) U_{\alpha_{2}}(1) \text {. }
$$

By (12.5) b) there is an element $g \in G$ such that $g$ interchanges $U_{r}(1)$ and $U_{\alpha_{2}}(1)$, and $g$ interchanges $U_{r_{17}}(1)$ and $U_{\alpha_{2}+2 \alpha_{3}}(1)$. Then $\left(U_{r}(1) U_{r_{17}}(1)\right)^{a \sigma_{\alpha_{3}}(1)}=$ $\left(U_{\alpha_{2}}(1) U_{\alpha_{2}+2 \alpha_{3}}(1)\right)^{\sigma_{\alpha_{3}}(1)}=U_{\alpha_{2}}(1) U_{\alpha_{2}+\alpha_{3}}(1)$ and by (3.1) $\left(U_{r}(1) U_{r_{17}}(1)\right)^{g \sigma_{\alpha_{3}}(1) U_{-\alpha_{3}}(\delta)}=$ $U_{\alpha_{2}+\alpha_{3}}(1) \sim U_{s}(1)$, where $\delta \in F_{8^{2}}$ satisfies $\delta+\delta^{q}=1$. Set $x=g U_{\alpha_{3}}(1) U_{-\alpha_{3}}(1)$. We have $\left(U_{r}(1) U_{r_{17}}(1) U_{\alpha_{2}+2 \alpha_{3}}(1)\right)^{x}=U_{\alpha_{2}+\alpha_{3}}(1) U_{r_{17}}(1)$. Conjugating this last element by $s_{1} s_{2} s_{3} s_{4} s_{2} s_{3} s_{2}$ we obtain $U_{\alpha}(1) U_{\beta}(1)$.

Finally consider the involution $y_{3}=U_{r}(1) U_{r_{17}}(1) U_{\alpha_{2}+2 \alpha_{3}}(1) U_{\alpha_{2}}(1)$. Conjugating by $U_{\alpha_{3}}(1) U_{-\alpha_{3}}(1) s_{2}$ we have $y_{3} \sim U_{r}(1) U_{r_{17}}(1) U_{\alpha_{3}}(1)=g$. Next conjugate $g$ by $U_{r_{4}}(1) U_{r_{1}}(1) s_{2} s_{1} s_{2} U_{\alpha_{2}}(1)$ to get

$$
g \sim U_{r_{8}}(1) U_{r_{8}}(1) U_{r_{5}}(1) .
$$

Then conjugate this by $s_{2} U_{\alpha_{3}}(1)$ to obtain

$$
g \sim U_{r_{9}}(1) U_{r_{6}}(1) U_{r_{14}}(1) \quad \text { (here use (3.1)(iv)) . }
$$

Next conjugate by $U_{r_{17}}(1) U_{r_{3}}(1)$ to get 


$$
g \sim U_{r_{9}}(1) U_{r_{\theta}}(1)
$$

Finally conjugation by $s_{3} s_{4} s_{2} s_{3} s_{2}$ gives

$$
g \sim U_{\alpha}(1) U_{\beta}(1) .
$$

This completes the proof of (12.7).

(12.8) Each involution in $E_{6}(q), q$ even, is conjugate to one of the following :

$$
\begin{aligned}
\text { i) } & x=U_{r}(1) \\
\text { ii) } & y=U_{\alpha}(1) U_{\beta}(1) \\
\text { iii) } & z=U_{r}(1) U_{\delta}(1) U_{。}(1) .
\end{aligned}
$$

Proof. We proceed as in (12.7). Here $P=P_{2}$ and $L=\left\langle U_{ \pm \alpha_{i}}: i \neq 2\right\rangle$ $\cong S L(6, q)$. The roots $t_{0}, \cdots, t_{k}$ are $t_{0}=r, t_{1}=r_{8}, t_{2}=r_{29}, t_{3}=r_{31}$. Consequently each involution in $G$ is conjugate to one of

$$
U_{r}(1), \quad U_{r}(1) U_{r_{8}}(1), \quad U_{r}(1) U_{r_{8}}(1) U_{r_{29}}(1), \quad U_{r}(1) U_{r_{8}}(1) U_{r_{29}}(1) U_{r_{31}}(1) .
$$

Conjugating $U_{r}(1) U_{r_{8}}(1)$ by $s_{2} s_{4} s_{3}$ we obtain $U_{\alpha}(1) U_{\beta}(1)$ and conjugating $U_{r}(1) U_{r_{8}}(1) U_{r_{29}}(1)$ by $s_{2} s_{4} s_{3} s_{6} s_{5}$ we obtain $U_{r}(1) U_{\delta}(1) U_{c}(1)$. It remains to consider

$$
g=U_{r}(1) U_{r_{8}}(1) U_{r_{29}}(1) U_{r_{31}}(1) .
$$

Conjugating $g$ by $s_{2} s_{3} s_{1} s_{6} s_{4} s_{3} s_{5}$ we obtain

$$
g \sim U_{r_{6}}(1) U_{r_{23}}(1) U_{r_{24}}(1) U_{r_{10}}(1) .
$$

Conjugating by $U_{\alpha_{1}}(1) U_{\alpha_{6}}(1)$ we have

$$
\begin{gathered}
g \sim U_{r_{6}}(1) U_{r_{7}}(1) U_{r_{23}}(1) U_{r_{7}}(1) U_{r_{24}}(1) U_{r_{9}}(1) U_{r_{25}}(1) U_{r_{10}}(1) U_{r_{10}}(1) \\
=U_{r_{6}}(1) U_{r_{23}}(1) U_{r_{9}}(1) U_{r_{25}}(1) U_{r_{24}}(1) .
\end{gathered}
$$

Next conjugate by $U_{\alpha_{4}}(1)$ to obtain

$$
\begin{gathered}
g \sim U_{r_{6}}(1) U_{r_{9}}(1) U_{r_{23}}(1) U_{r_{25}}(1) U_{r_{9}}(1) U_{r_{28}}(1) U_{r_{24}}(1) \\
=U_{r_{6}}(1) U_{r_{23}}(1) U_{r_{9}}(1) U_{r}(1) U_{r_{25}}(1) U_{r_{9}}(1) U_{r_{25}}(1) U_{r_{24}}(1) \\
=U_{r_{6}}(1) U_{r_{23}}(1) U_{r}(1) U_{r_{24}}(1) .
\end{gathered}
$$

Then conjugation by $U_{r_{25}}(1)$ we have

$$
g \sim U_{r_{8}}(1) U_{r_{23}}(1) U_{r_{24}}(1) .
$$

Finally conjugation by $s_{1} s_{4} s_{6} s_{5} s_{3} s_{4}$ shows that 


$$
g \sim U_{r}(1) U_{\delta}(1) U_{\epsilon}(1) .
$$

This completes this proof of (12.8).

(12.9) Each involution in $E_{7}(q), q$ even, is conjugate to one of the following :
i) $x=U_{r}(1)$
ii) $y=U_{\alpha}(1) U_{\beta}(1)$
iii) $z=U_{\gamma}(1) U_{\delta}(1) U_{s}(1)$
iv) $u=U_{\varphi}(1) U_{\psi}(1) U_{\theta}(1)$
v) $v=U_{r}(1) U_{\theta}(1) U_{\psi}(1) U_{\varphi}(1)$.

In the proof of (12.9) we will use (12.4) as usual, and consequently we need the involutions in $L=L_{1} \cong S O^{+}(12, q)$. We have

(12.10) Let $G=E_{7}(q)$ and $L_{1}=\left\langle U_{ \pm \alpha_{i}}: i \neq 1\right\rangle$. Then each involution in $L_{1}$ is conjugate to one of the following:
i) $U_{r_{15}}(1)$
ii) $U_{r_{10}}(1) U_{r_{37}}(1)$
iii) $U_{r_{5}}(1) U_{r_{7}}(1)$
iv) $U_{r_{38}}(1) U_{r_{4}}(1) U_{r_{8}}(1)$
v) $U_{r_{36}}(1) U_{\alpha_{33}}(1) U_{r_{5}}(1)$
vi) $U_{r_{38}}(1) U_{r_{33}}(1) U_{r_{7}}(1)$
vii) $U_{\alpha_{3}}(1) U_{r_{35}}(1) U_{r_{15}}(1) U_{\alpha_{5}}(1)$.

Proof. $L_{1}$ has root diagram of type $D_{6}$ with fundamental system $\left\{\alpha_{2}, \alpha_{3}, \alpha_{4}, \alpha_{5}, \alpha_{6}, \alpha_{7}\right\}$. The highest root is $U_{r_{15}}=Z\left(U \cap L_{1}\right)$ and $N_{L_{1}}\left(U_{r_{15}}\right)=$ $Q_{0} L_{0} H_{0}$, where $Q_{0}=O_{2}\left(Q_{0} L_{0} H_{0}\right), H_{0}=H \cap L_{1}$, and $L_{0}=\left\langle U_{ \pm \alpha_{i}}: i \neq 1,6\right\rangle=$ $\left\langle U_{ \pm \alpha_{i}}: i=2,3,4,5\right\rangle \times\left\langle U_{ \pm \alpha_{7}}\right\rangle \cong S O^{+}(8, q) \times S L(2, q)$. Consequently each involution in $L_{1}$ is conjugate to an involution in $L_{0} \times\left\langle U_{ \pm r_{15}}\right\rangle$. We first find the involutions in $\left\langle U_{ \pm \alpha_{i}}: i=2,3,4,5\right\rangle \cong S O^{+}(8, q)$. For this we again use (12.4) noting that the highest root is $U_{r_{35}}$ and the corresponding Levi factor is $\left\langle U_{ \pm \alpha_{2}}\right\rangle \times\left\langle U_{ \pm \alpha_{3}}\right\rangle \times\left\langle U_{ \pm \alpha_{5}}\right\rangle$. Conjugating by elements of $\left\langle s_{2}, \cdots, s_{5}\right\rangle$ we obtain the fact that each involution in $\left\langle U_{ \pm \alpha_{i}}: i=2,3,4,5\right\rangle$ is conjugate to one of

$$
\begin{gathered}
U_{r_{35}}(1), \quad U_{\alpha_{3}}(1) U_{r_{35}}(1), \quad U_{\alpha_{5}}(1) U_{r_{35}}(1), \\
U_{\alpha_{2}}(1) U_{r_{35}}(1), \quad U_{\alpha_{3}}(1) U_{\alpha_{5}}(1) U_{r_{35}}(1) .
\end{gathered}
$$

(For this use the fact that $\left(U_{\alpha_{2}}(1) U_{\alpha_{3}}(1) U_{\alpha_{5}}(1) U_{r_{35}}(1)\right)^{g}=U_{\alpha_{2}}(1) U_{\alpha_{3}}(1) U_{\alpha_{5}}(1)$, where $g=U_{\alpha_{4}+\alpha_{5}}(1) U_{\alpha_{3}+\alpha_{4}}(1) U_{\alpha_{2}+\alpha_{4}}(1) U_{\alpha_{3}+\alpha_{4}+\alpha_{5}}(1)$.) Consequently each involution in $L_{1}$ is conjugate to one of 


$$
\begin{aligned}
& U_{r_{35}}(1), \quad U_{\alpha_{3}}(1) U_{r_{35}}(1), \quad U_{\alpha_{5}}(1) U_{r_{35}}(1), \quad U_{\alpha_{2}}(1) U_{r_{35}}(1), \\
& U_{\alpha_{3}}(1) U_{\alpha_{5}}(1) U_{r_{35}}(1), \quad U_{r_{35}}(1) U_{r_{15}}(1), \quad U_{\alpha_{3}}(1) U_{r_{35}}(1) U_{r_{15}}(1), \\
& U_{\alpha_{5}}(1) U_{r_{35}}(1) U_{r_{15}}(1), \quad U_{\alpha_{2}}(1) U_{r_{35}}(1) U_{r_{15}}(1), \\
& U_{\alpha_{3}}(1) U_{\alpha_{5}}(1) U_{r_{35}}(1) U_{r_{15}}(1) .
\end{aligned}
$$

We easily have $U_{r_{35}}(1) \sim U_{r_{15}}(1)$. Conjugating $U_{\alpha_{3}}(1) U_{r_{35}}(1)$ by $s_{6} s_{5} s_{4} s_{2} s_{7} s_{6} s_{5}$ $s_{4} s_{6} s_{7}, U_{\alpha_{2}}(1) U_{r_{35}}(1)$ by $s_{6} s_{7} s_{5} s_{4} s_{3} s_{6} s_{5} s_{4} s_{6} s_{5}$, and $U_{r_{35}}(1) U_{r_{15}}(1)$ by $s_{6} s_{5}$ we see that each of these elements is conjugate to $U_{r_{10}}(1) U_{r_{37}}(1)$. Conjugating $U_{\alpha_{5}}(1) U_{r_{35}}(1)$ by $s_{6} s_{7} s_{4} s_{3}$ we have this element conjugate to $U_{r_{5}}(1) U_{r_{7}}(1)$. Next conjugate $U_{\alpha_{3}}(1) U_{\alpha_{5}}(1) U_{r_{35}}(1)$ by $s_{6} s_{5} s_{4} s_{2} s_{7} s_{5} s_{6} s_{4}$ and obtain $U_{r_{38}}(1) U_{r_{4}}(1)$ $U_{r_{8}}(1)$. Conjugate $U_{\alpha_{3}}(1) U_{r_{35}}(1) U_{r_{15}}(1)$ by $s_{6} s_{5} s_{4} s_{2} s_{5} s_{4} s_{6}$ and get this element conjugate to $U_{r_{36}}(1) U_{r_{3 s}}(1) U_{r_{5}}(1)$. Next conjugate $U_{\alpha_{5}}(1) U_{r_{35}}(1) U_{r_{15}}(1)$ by $s_{6} s_{7} s_{4} s_{5}$ and get $U_{r_{36}}(1) U_{r_{4}}(1) U_{r_{8}}(1)$, and conjugate $U_{\alpha_{2}} U_{r_{35}} U_{r_{15}}$ by $s_{4} s_{6} s_{5} s_{7} s_{4} s_{3} s_{7}$ getting $U_{r_{38}}(1) U_{r_{7}}(1) U_{r_{33}}(1)$.

We now prove (12.9). Using (12.4) we have each involution in $G=E_{7}(q)$ conjugate to one in $L_{1} U_{r}(1)$. Consequently each involution in $G$ is conjugate to $g r$ for $g$ one of the involutions in (12.10). We must show that each of these is conjugate to one of $x, y, z, u, v$.

Conjugating $U_{r_{15}}(1) U_{r}(1)$ by $s_{1} s_{3} s_{4} s_{2}$ and $U_{r_{10}}(1) U_{r_{37}}(1) U_{r}(1)$ by $s_{1} s_{3} s_{4} s_{5} s_{2} s_{6}$, we see that $U_{r_{15}}(1) U_{r}(1) \sim y$ and $U_{r_{10}}(1) U_{r_{37}}(1) U_{r}(1) \sim z$. Next conjugate $U_{r_{5}}(1) U_{r_{7}}(1) U_{r}(1)$ by $s_{1} s_{3} s_{4} s_{2} s_{5} s_{4} s_{6} s_{5}$ to obtain $u$. Transforming $U_{r_{38}}(1) U_{r_{4}}(1)$ $U_{r_{8}}(1) U_{r}(1)$ and $U_{r_{36}}(1) U_{r_{33}}(1) U_{r_{7}}(1) U_{r}(1)$ by $s_{1} s_{3} s_{5} s_{4} s_{2} s_{5} s_{4} s_{5} s_{6} s_{3}$ and $s_{1} s_{3} s_{4} s_{5} s_{6} s_{7} s_{3}$, respectively, we check that both of these involutions are conjugate to $v$.

We claim that $g=U_{r_{36}}(1) U_{r_{33}}(1) U_{r_{5}}(1) U_{r}(1)$ is conjugate to $z$. To see this first conjugate by $s_{1} s_{3} s_{4} s_{5} s_{2} s_{6} s_{5} s_{4}$ to get

$$
g \sim U_{r_{45}}(1) U_{r_{44}}(1) U_{r_{11}}(1) U_{r_{16}}(1) .
$$

Conjugate this by $U_{\alpha_{7}}(1) U_{\alpha_{5}}(1)$ and obtain

$$
g \sim U_{r_{45}}(1) U_{r_{48}}(1) U_{r_{14}}(1) U_{r_{44}}(1) U_{r_{11}}(1) .
$$

Then conjugate by $U_{\alpha_{3}}(1)$ to obtain

$$
\begin{gathered}
g \sim U_{r_{45}}(1) U_{r_{48}}(1) U_{r_{14}}(1) U_{r_{44}}(1) U_{r_{48}}(1) U_{r_{11}}(1) U_{r_{14}}(1) \\
=U_{r_{45}}(1) U_{r_{48}}(1) U_{r_{44}}(1) U_{r_{14}}(1) U_{r}(1) U_{r_{46}}(1) U_{r_{11}}(1) U_{r_{14}}(1) \\
=U_{r_{45}}(1) U_{r_{44}}(1) U_{r}(1) U_{r_{11}}(1)
\end{gathered}
$$

Finally conjugating by $U_{r_{48}}(1) s_{7} s_{3} s_{5} s_{4} s_{2} s_{6} s_{5} s_{4} s_{3}$ gives $g \sim z$.

The last case is $g=U_{\alpha_{3}}(1) U_{r_{35}}(1) U_{r_{15}}(1) U_{\alpha_{5}}(1) U_{r}(1)$. Conjugate $g$ by 
the element $s_{1} s_{6} s_{4} s_{2} s_{3} s_{4} s_{5} s_{4} s_{2} s_{3} s_{7} s_{6} s_{7} s_{4} s_{5} s_{3} s_{4} s_{2}$ above to get

$$
g \sim v U_{\alpha_{1}+\alpha_{3}+\alpha_{4}+\alpha_{5}+\alpha_{8}}(1) .
$$

Then conjugating by $s_{2} s_{4} U_{\alpha_{7}}(1)$ we have

$$
g \sim U_{r_{42}}(1) U_{r_{11}}(1) U_{r_{13}}(1) U_{r_{14}}(1) U_{r_{15}}(1) U_{r_{46}}(1) U_{r_{16}}(1) .
$$

Conjugation by $U_{\alpha_{5}}(1)$ then gives

$$
\begin{gathered}
g \sim U_{r_{42}}(1) U_{r_{44}}(1) U_{r_{11}}(1) U_{r_{13}}(1) U_{r_{13}}(1) U_{r_{14}}(1) U_{r_{18}}(1) U_{r_{15}}(1) U_{r_{46}}(1) U_{r_{16}}(1) \\
=U_{r_{42}}(1) U_{r_{44}}(1) U_{r_{11}}(1) U_{r_{14}}(1) U_{r_{15}}(1) U_{r_{48}}(1) .
\end{gathered}
$$

Next conjugate by $U_{\alpha_{3}}(1)$ and get

$$
\begin{gathered}
g \sim U_{r_{42}}(1) U_{r_{45}}(1) U_{r_{44}}(1) U_{r_{46}}(1) U_{r_{11}}(1)_{r_{14}}(1) U_{r_{14}}(1) U_{r_{15}}(1) U_{r_{46}}(1) \\
=\left(U_{r_{42}}(1) U_{r_{45}}(1) U_{r_{44}}(1) U_{r_{11}}(1) U_{r_{15}}(1)\right)^{U_{r_{46}}(1)}
\end{gathered}
$$

Consequently $g \sim U_{r_{42}}(1) U_{r_{45}}(1) U_{r_{44}}(1) U_{r_{11}}(1) U_{r_{15}}(1)$ and conjugation by $U_{-\alpha_{7}}(1)$ and then by $s_{5} s_{7} s_{3} s_{4} s_{2}$ gives $g \sim v$. This completes the proof of (12.9).

(12.11) Each involution in $E_{8}(q), q$ even, is conjugate to one of the following:

$$
\begin{aligned}
\text { i) } & x=U_{r}(1) \\
\text { ii) } & y=U_{\alpha}(1) U_{\beta}(1) \\
\text { iii) } & z=U_{r}(1) U_{\delta}(1) U_{s}(1) \\
\text { iv) } & u=U_{\varphi}(1) U_{\psi}(1) U_{\theta}(1) U_{\omega}(1) .
\end{aligned}
$$

Proof. For $G=E_{8}(q)$ we have $P=P_{7}$ and $L=L_{7} \cong E_{7}(q)$. By (12.4) each involution in $G$ is conjugate to one in $L_{7} U_{r}(1)$, so each involution in $G$ is conjugate to one of

$$
\begin{aligned}
& U_{r}(1), \quad U_{s_{27}}(1) U_{r}(1), \quad U_{s_{21}}(1) U_{s_{23}}(1) U_{r}(1), \\
& U_{s_{48}}(1) U_{s_{18}}(1) U_{s_{19}}(1) U_{r}(1), \quad U_{s_{13}}(1) U_{s_{14}}(1) U_{s_{15}}(1) U_{r}(1), \\
& U_{s_{48}}(1) U_{s_{13}}(1) U_{s_{14}}(1) U_{s_{15}}(1) U_{r}(1),
\end{aligned}
$$

where our notation is combining that of Tables 3 and 4 . We will write $U_{s_{i}}$ rather than $U_{r_{i}}$ to indicate we are using Table 3 for $E_{7}$. For example $s_{28}=\alpha_{2}$.

Conjugating $U_{s_{27}}(1) U_{r}(1)$ by $s_{8} s_{7} s_{6} s_{5} s_{4} s_{2}$ we see that this involution is conjugate to $y$. Then conjugate $U_{s_{21}}(1) U_{s_{23}}(1) U_{r}(1)$ by $s_{8} s_{7} s_{6} s_{5} s_{4} s_{2} s_{3} s_{1} s_{4} s_{5}$, obtaining $z$. 
Next we claim that $g=U_{s_{13}}(1) U_{s_{14}}(1) U_{s_{15}}(1) U_{r}(1) \sim z$. To see this first conjugate by $s_{8} s_{7} s_{6} s_{5} s_{4} s_{3} s_{1} s_{2} s_{4} s_{5} s_{6} s_{3} s_{4}$ getting

$$
g \sim U_{r_{22}}(1) U_{r_{34}}(1) U_{r_{24}}(1) U_{r_{37}}(1) .
$$

Conjugate this element $U_{\alpha_{7}}(1) U_{\alpha_{2}}(1)$ to get

$$
g \sim U_{r_{22}}(1) U_{r_{25}}(1) U_{r_{38}}(1) U_{r_{34}}(1) U_{r_{24}}(1) .
$$

Then conjugate by $U_{\alpha_{5}}(1)$ and obtain

$$
\begin{gathered}
g \sim U_{r_{22}}(1) U_{r_{25}}(1) U_{r_{34}}(1) U_{r_{24}}(1) U_{r_{25}}(1) \\
=U_{r_{22}}(1) U_{r_{34}}(1) U_{r}(1) U_{r_{24}}(1) .
\end{gathered}
$$

Now conjugate by $U_{r_{35}}(1)$ to get

$$
g \sim U_{r_{22}}(1) U_{r_{34}}(1) U_{r_{24}}(1),
$$

and then by $s_{7} s_{2} s_{5} s_{6} s_{4} s_{3} s_{1} s_{5} s_{4} s_{3} s_{2} s_{4} s_{5} s_{6} s_{7}$ to get the claim.

The last case is $U_{s_{48}}(1) U_{s_{13}}(1) U_{s_{14}}(1) U_{s_{15}}(1) U_{r}(1)=g U_{s_{48}}(1)$ where $g$ is as in the preceding paragraph. The series of conjugations leading to $g \sim z$ when applied to $g U_{s_{48}}(1)$ yields

$$
g U_{s_{48}}(1) \sim z U_{s_{48}}(1) .
$$

From here conjugate by $s_{7} s_{6} s_{5} s_{4} s_{3} s_{1}$ and get $g U_{s_{48}}(1) \sim u$. This completes the proof of (12.10).

Section 13. Centralizers of involutions in $\boldsymbol{F}_{4}(q), q$ even.

Let $G=F_{4}(q)$ with $q$ a power of 2 . Then $W=\left\langle s_{1}, s_{2}, s_{3}, s_{4}\right\rangle$ and the action on $\left\{\alpha_{1}, \alpha_{2}, \alpha_{3}, \alpha_{4}\right\}$ is as follows

$$
\begin{aligned}
& \left(\alpha_{j}\right) s_{i}=\alpha_{j} \\
& \left(\alpha_{i}\right) s_{i}=-\alpha_{i} . \\
& \left(\alpha_{j}\right) s_{i}=\alpha_{j}+\alpha_{i} \\
& \left(\alpha_{2}\right) s_{3}=\alpha_{2}+2 \alpha_{3} .
\end{aligned} \quad \text { if } \quad|i-j| \geq 2 .
$$

We will distinguish the roots $r=2342, s=1232, \alpha=1221$, and $\beta=$ 1242. Then $r$ and $\beta$ are long roots (hence conjugate under $W$ ) and $s$ and $\alpha$ are short roots (and conjugate under $W$ ). Also $r$ is the root of highest height.

In [14] Guterman determined the four conjugacy classes of involutions in $F_{4}(q)$ and their centralizers. So we simply use his results together 
with some necessary additional information concerning structural properties of the centralizers. From (12.6) we have

(13.1) $F_{4}(q)$ has four conjugacy classes of involutions with representatives given by
i) $t=U_{r}(1)$
ii) $u=U_{s}(1)$
iii) $t u=U_{r}(1) U_{s}(1)$
iv) $v=U_{\alpha}(1) U_{\beta}(1)$.

We next discuss the centralizers of these involutions. $C_{G}(t)=O^{2^{\prime}}\left(P_{1}\right)$ $=Q_{1}\left\langle U_{ \pm \alpha_{i}}: i=2,3,4\right\rangle=Q_{1} L_{1}$, where $L_{1} \cong S p(6, q)$. Moreover $Z\left(C_{G}(t)\right)=$ $U_{r}=Q_{1}^{\prime}, Q_{1} \leq C_{G}(t)^{\prime}$, and $P_{1}$ contains a subgroup of order $q-1$ transitive on $U_{r}^{\sharp}$. (The structure of $P_{1}$ is discussed in [7], Section 4.)

The graph automorphism interchanges $r$ and $s$, fusing $t$ and $u$. Consequently $C_{G}(u)=O^{2^{\prime}}\left(P_{4}\right)=Q_{4}\left\langle U_{ \pm \alpha_{i}}: i=1,2,3\right\rangle=Q_{4} L_{4}$, where $L_{4} \cong$ $S p(6, q)$. Also $Z\left(C_{G}(U)\right)=U_{s}=Q_{4}^{\prime}, Q_{4} \leq C_{G}(u)^{\prime}$, and $P_{4}$ contains a subgroup of order $q-1$ transitive on $U_{s}^{\#}$.

Next consider $t u$, with centralizer $C_{G}(t u)=O^{2^{\prime}}\left(P_{1,4}\right)=Q_{1} Q_{4}\left\langle U_{ \pm \alpha_{2}}, U_{ \pm \alpha_{3}}\right\rangle$ $=Q_{1} Q_{4} L_{14}$, where $L_{14} \cong S p(4, q)$. Using Table 1 together with (3.1) we show that $\left(Q_{1} Q_{4}\right)^{\prime}=U_{r_{10}} U_{r_{11}} U_{r_{12}} U_{r_{13}} \times U_{r} U_{s}$ and $\left(Q_{1} Q_{4}\right)^{\prime}=Z\left(Q_{1} Q_{4}\right)$.

Now (3.1) shows that $\left\langle U_{ \pm \alpha_{3}}\right\rangle=\left\langle U_{\alpha_{3}}, s_{3}\right\rangle \cong S L(2, q)$ acts on $U_{r_{10}} U_{r_{11}}$ as it does on its natural 2-dimensional module over $\boldsymbol{F}_{q}$. Similarly $\left\langle U_{ \pm \alpha_{2}}\right\rangle$ $\cong S L(2, q)$ acts irreducibly on $U_{r_{12}} U_{r_{13}}$. Consequently $Z\left(C_{G}(t u)\right) \cap\left(Q_{1} Q_{4}\right)^{\prime}$ $=U_{r} U_{s}$. Consider the elementary abelian group $Q_{1} Q_{4} /\left(Q_{1} Q_{4}\right)^{\prime}$, which is acted on by $L_{1,4}$. Using the action of the groups $\left\langle U_{ \pm \alpha_{2}}\right\rangle \cong S L(2, q) \cong$ $\left\langle U_{ \pm \alpha_{3}}\right\rangle$ we see that in this action the trivial space for $L_{1,4}$ is the image of $\left\langle U_{r_{9}}, U_{r_{17}}\right\rangle$. Moreover $L_{1,4}$ does centralize $\left\langle U_{r_{9}}, U_{r_{17}}\right\rangle$. However $\left[Q_{1} Q_{4}, L_{14}\right]=Q_{1} Q_{4}$.

We make one final observation concerning $C_{G}(t u)$. Suppose $P$ is parabolic and contains $C_{G}(t u)$. Then considering $P / O_{2}(P)$ we see that $P \sim P_{1}, P_{4}$, or $P_{14}$. On the other hand $U \leq C_{G}(t) \leq P$, so Lemma 1.6 of [19] implies that $P=P_{1}, P_{4}$ or $P_{14}$. So $C_{G}(t u)$ is contained in only the parabolic subgroups $P_{1}, P_{4}, P_{14}$.

Next we consider $C_{G}(v)$. Guterman proves that $C_{G}(v)=\left\langle U_{ \pm \alpha_{1}}\right.$, $\left.U_{ \pm \alpha_{4}}, U_{r}: r>0, r \neq \alpha_{2}, \alpha_{3}, \alpha_{1}+\alpha_{2}, \alpha_{3}+\alpha_{4}\right\rangle$. Then $C_{G}(v) \leq P_{2,3}$, covers $O^{2^{\prime}}\left(P_{2,3} / O_{2}\left(P_{2,3}\right)\right)$, and setting $U_{0}=C_{G}(v) \cap O_{2}\left(P_{2,3}\right)$, we have $U_{0}=O_{2}\left(C_{G}(v)\right)$, $\left|O_{2}\left(P_{2,3}\right): U_{0}\right|=q^{4}$, and $C_{G}(v)=U_{0}\left(\left\langle U_{ \pm \alpha_{1}}\right\rangle \times\left\langle U_{ \pm \alpha_{4}}\right\rangle\right)$. 
We will need information concerning the structure of $U_{0}$ and the action of $L_{2,3}=\left\langle U_{ \pm \alpha_{1}}\right\rangle \times\left\langle U_{ \pm \alpha_{4}}\right\rangle$ on $U_{0}$. First we note that $U_{0}=$ $\left\langle U_{r}: r \in \mathscr{L}^{2} \cup \mathscr{L}^{3}, r \neq \alpha_{2}, \alpha_{3}, \alpha_{1}+\alpha_{2}, \alpha_{3}+\alpha_{4}\right\rangle$. Next we use (3.1) and Table 1 to determine $U_{0}^{\prime}$ and $Z\left(U_{0}\right)$. For example $\left[U_{r_{18}}, U_{r_{6}}\right]=U_{r_{8}},\left[U_{r_{20}}, U_{r_{11}}\right]$ $=U_{r_{14}},\left[U_{r_{3}}, U_{r_{6}}\right]=U_{r_{24}},\left[U_{r_{11}}, U_{r_{19}}\right]=U_{r_{13}},\left[U_{r_{5}}, U_{r_{16}}\right]=U_{r_{7}}=U_{\beta},\left[U_{r_{9}}, U_{r_{20}}\right]$ $=U_{r_{12}}=U_{\alpha}$. So each of $U_{\alpha}, U_{\beta}, U_{r_{8}}, U_{r_{13}}, U_{r_{14}}$, and $U_{r_{24}}$ are in $U_{0}^{\prime}$. Moreover we see that each of these is in $Z\left(U_{0}\right)$ It is then easy to check that $U_{0}^{\prime}=Z\left(U_{0}\right)=U_{\alpha} U_{\beta} \times U_{r_{8}} U_{r_{13}} U_{r_{14}} U_{r_{24}}$ and that $\left[U_{0}, L_{2,3}\right]=U_{0}$. Also (3.1)(iii) shows that $U_{\alpha} U_{\beta} \leq Z\left(C_{G}(v)\right.$ ), whereas (3.1)(ii) shows that $\left\langle U_{ \pm \alpha_{1}}\right\rangle$ acts irreducibly on $U_{r_{8}} \times U_{r_{24}}$ (which affords the natural module for $S L(2, q))$, and that $\left\langle U_{ \pm \alpha_{4}}\right\rangle$ acts irreducibly on $U_{r_{13}} \times U_{r_{14}}$.

Assume $q>2$. Then using (3.1) and Table 1 we have $\left[U_{\alpha_{1}}, U_{r_{16}}\right]=$ $U_{r_{3}}$, so $U_{r_{3}}$ and $U_{r_{3}}^{s_{4}}=U_{r_{5}}$ are in $C_{G}(v)^{\prime}$. Similarly $\left[U_{\alpha_{4}}, U_{r_{4}}\right]=U_{r_{12}} U_{r_{6}}$, so $U_{r_{12}}, U_{r_{6}}, U_{r_{6}}^{s_{4}}=U_{r_{4}}$ are all in $C_{G}(v)^{\prime}$. Continuing we see that $U_{r} \leq$ $C_{G}(v)^{\prime}$ for each root $r \in \Delta^{+}$satisfying $U_{r} \leq U_{0}$. In particular $C_{G}(v)=$ $O^{2}\left(C_{G}(v)\right)=C_{G}(v)^{\prime}$.

Continue the assumption that $q>2$. It is easy to see that there is a subgroup $H_{0} \leq H$ such that $H_{0}=H_{1} \times H_{2}, H_{1} \cong H_{2}$ is cyclic of order $q-1, H_{1}$ centralizes $U_{\alpha}$ and is fixed-point free on $U_{\beta}$, while $H_{2}$ centralizes $U_{\beta}$ and is fixed-point-free on $U_{\alpha}$. For example this can be seen by observing that $\left\langle U_{ \pm \alpha}, U_{ \pm \beta}\right\rangle=\left\langle U_{ \pm \alpha}\right\rangle \times\left\langle U_{ \pm \beta}\right\rangle \equiv S L(2, q) \times S L(2, q)$.

We claim that the set $U_{\alpha}^{\sharp} U_{\beta}^{\sharp}$ is uniquely determined by the abstract structure of the group $C_{G}(v)$. Recall that $Z\left(U_{0}\right)=U_{\alpha} U_{\beta} \times Y$ with $Y \unlhd C_{G}(v)$ and $U_{\alpha} U_{\beta}=Z\left(C_{G}(v)\right)$. So $U_{\alpha} U_{\beta}$ is uniquely determined. Consider $\bar{U}_{0}=U_{0} / Y$ and let bars denote images. Using (3.1) we have $\bar{U}_{0}=$ $B_{1} \times B_{2} \times B_{3}$, where $B_{3}$ is elementary and $B_{1}, B_{2}$ are both special of order $q^{5}$ with respective centers $\bar{U}_{\alpha}$ and $\bar{U}_{\beta}$. In fact for $i=1,2 B_{i}=$ $\left\langle X_{i}, Y_{i}\right\rangle$, where $X_{i}$ and $Y_{i}$ are elementary of order $q^{2}$ and for each $x_{i} \in X_{i}^{\sharp},\left|C_{Y_{i}}\left(x_{i}\right)\right|=q$. It is then easy to describe the elements of order 4 in $\bar{U}_{0}$ and to compute their squares. We conclude that the elements in $\overline{U_{\alpha}^{\sharp} U_{\beta}^{\sharp}}$ each have the same number of square roots, and this number is different from the number of square roots of an element in $\overline{U_{\alpha}^{\#} \cup U_{\beta}^{\sharp}}$. This proves the claim.

Finally we claim that the only maximal parabolic subgroups of $G$ containing $C_{G}(v)$ are $P_{3}, P_{4}, P_{1}^{s_{1} s_{2}}$, and $P_{4}^{s_{4} s_{3}}$. We first check that $C_{G}(v) \leq$ $P_{1}^{s_{1} s_{2}}$ and $P_{4}^{s_{4} s_{3}}$. This is easily done by showing that $U_{0} \leq Q_{1}^{s_{1} s_{2}} \cap Q_{1}^{s_{4} s_{3}}$ and noting that $L_{2,3} \leq L_{1}^{s_{1} s_{2}} \cap L_{4}^{s_{4} s_{3}}$. 
Suppose $C_{G}(v) \leq P_{1}^{g}$ some $g$. First assume that $U_{0} \cap O_{2}\left(P_{1}^{g}\right)^{\prime}=1$. Then $U_{0} \cap O_{2}\left(P_{1}^{g}\right)$ is abelian. The results in [7] Section 4 show that $\left|U_{0} \cap O_{2}\left(P_{1}^{g}\right)\right| \leq q^{10}$, so that $\left|U_{0} O_{2}\left(P_{1}^{g}\right) / O_{2}\left(P_{1}^{g}\right)\right| \geq q^{8}$. On the other hand $L_{1}^{g} \cong S p(6, q)$ and $C_{G}(v) O_{2}\left(P_{1}^{g}\right) / O_{2}\left(P_{1}^{g}\right)$ is contained in a proper parabolic subgroup of $P_{1}^{g} / O_{2}\left(P_{1}^{g}\right)$ that has a section isomorphic to $\left\langle U_{ \pm \alpha_{1}}\right\rangle \times\left\langle U_{ \pm \alpha_{4}}\right\rangle$ $=S L(2, q) \times S L(2, q)$. This is impossible. Consequently $U_{0} \cap O_{2}\left(P_{1}^{g}\right)^{\prime} \neq 1$ and $U_{r}^{g} \cap U_{0} \neq 1$. Now $C_{G}(v) \leq O^{2^{\prime}}\left(P_{1}^{g}\right)=C\left(U_{r}^{g}\right)$, so $U_{r}^{g} \cap U_{0} \leq Z\left(C_{G}(v)\right)$ and consequently $U_{r}^{g}=U_{\beta}$. As $r=\beta^{s_{2} s_{1}}$, we have $g \in P_{1} s_{1} s_{2}$ and $P_{1}^{g}=P_{1}^{s_{1} s_{2}}$. Similarly (by applying a graph automorphism) if $C_{G}(v) \leq P_{4}^{g}$, then $P_{4}^{g}=$ $P_{4}^{s_{4} s_{3}}$.

Next suppose $C_{G}(v) \leq P_{2}^{g}$. We consider two cases. First assume that $U_{0} \leq O_{2}\left(P_{2}^{g}\right)$. From Table 1 and (3.1) we see that $Q_{2}$ has class 3 with $\left[Q_{2}, Q_{2}, Q_{2}\right]=U_{r_{8}} U_{r_{24}}$ and in $P_{2},\left\langle U_{ \pm \alpha_{1}}\right\rangle$ acts irreducibly on $\left[Q_{2}, Q_{2}, Q_{2}\right]$. Now $L_{2} \cong S L(2, q) \times S L(3, q)$. So $C_{G}(v)$ must cover $\left\langle U_{ \pm \alpha_{1}}\right\rangle^{g}$ if $\left.q\right\rangle 2$ and $O_{3}\left(\left\langle U_{ \pm \alpha_{1}}\right\rangle^{g}\right)$ if $q=2$. In either case $C_{G}(v)$ acts irreducibly on $\left[Q_{2}, Q_{2}, Q_{2}\right]^{g}$. Since $\left|U_{0}\right|=q^{18}$ and $\left|Q_{2}^{g}\right|=q^{20}$, we must have $\left[Q_{2}, Q_{2}, Q_{2}\right] \leq U_{0}$ and hence in $Z\left(U_{0}\right)$ and normal in $C_{G}(v)$. It then follows that $\left[Q_{2}, Q_{2}, Q_{2}\right]^{g}=U_{r_{8}} U_{r_{24}}$, $g \in N\left(U_{r_{8}} U_{r_{24}}\right)=P_{2}$. Now assume that $U_{0} \not O_{2}\left(P_{2}^{q}\right)$, so that $C_{G}(v) Q_{2}^{g} / Q_{2}^{g}$ is contained in a parabolic subgroup of $P_{2}^{g} / Q_{2}^{g}$. This group has the form $P_{2}^{g} \cap P_{i}^{g x}$ for $x \in P_{2}^{g}$. As $S L(2, q) \times S L(2, q)$ is involved in $C_{G}(v), i=3$ or 4 . Now $g x=p_{2} g$ for some $p_{2} \in P_{2}$, so $\left(P_{2}^{g} \cap P_{i}^{g x}\right)=\left(P_{2} \cap P_{i}\right)^{p_{2} g}$. If $i=3$, then since $P_{2} \cap P_{3}=N_{G}\left(\left(O_{2}\left(P_{2} \cap P_{3}\right)\right)^{\prime}\right)=N_{G}\left(U_{0}^{\prime}\right)$ and $U_{0} \leq O_{2}\left(P_{2}^{g} \cap P_{3}^{g x}\right)$, we have $P_{2} \cap P_{3}=\left(P_{2} \cap P_{3}\right)^{p_{2} g}, p_{2} g \in P_{2} \cap P_{3}, g \in P_{2}$, as desired. If $i=4$, then $C_{G}(v) \leq\left(P_{2} \cap P_{4}\right)^{p_{2} g} \leq P_{4}^{s_{4} s_{3}}$. But then $\left(P_{2} \cap P_{4}\right)^{p_{2} g}$ is a parabolic subgroup of $P_{4}^{s_{4} s_{3}}$ and it is easy to see that this must be $\left(P_{2} \cap P_{4}\right)^{s_{4} s_{3}}$. Consequently $\left(P_{2} \cap P_{4}\right)^{p_{2} g}=\left(P_{2} \cap P_{4}\right)^{s_{4} s_{3}}$ and $p_{2} g s_{3} s_{4} \in P_{2} \cap P_{4}$. Again $g \in P_{2}$. So in all cases $P_{2}^{g}=P_{2}$. Similarly we show that $C_{G}(v) \leq P_{3}^{g}$ implies $P_{3}^{g}=$ $P_{3}$. This proves the claim.

We have now proved

(13.2) The maximal parabolic subgroups of $G$ containing the centralizers of the involutions in (13.1) are as follows:
i) $C_{G}(t) \leq P_{1}$
ii) $C_{G}(u) \leq P_{4}$
iii) $C_{G}(t u) \leq P_{1}, P_{4}$
iv) $C_{G}(v) \leq P_{2}, P_{3}, P_{1}^{s_{1} s_{2}}, P_{4}^{s_{4} s_{3}}$.

(13.3) With notation as in (13.1) we have 
i) $C_{G}(t)=\left(C_{G}(t)\right)^{\prime}=O^{2^{\prime}}\left(P_{1}\right) . \quad Z\left(C_{G}(t)\right)=Q_{1}^{\prime}=U_{r}$.

ii) $C_{G}(u)=\left(C_{G}(u)\right)^{\prime}=O^{2^{\prime}}\left(P_{4}\right) . \quad Z\left(C_{G}(u)\right)=Q_{4}^{\prime}=U_{s}$.

iii) $C_{G}(t u)=O^{2^{\prime}}\left(P_{14}\right)=Q_{1} Q_{4} L_{1,4}$ and $Q_{1} Q_{4}=\left[Q_{1} Q_{4}, L_{1,4}\right]$.

$$
Z\left(Q_{1} Q_{s}\right)=\left(Q_{1} Q_{4}\right)^{\prime} \quad \text { and } \quad Z\left(C_{G}(t u)\right)=U_{r} U_{s}
$$

iv) $C_{G}(v)=\left\langle U_{ \pm \alpha_{1}}, U_{ \pm \alpha_{4}}, U_{r}: r>0, r \neq \alpha_{2}, \alpha_{3}, \alpha_{1}+\alpha_{2}, \alpha_{3}+\alpha_{4}\right\rangle=U_{0} L_{2,3}$, where $U_{0}=O_{2}\left(C_{G}(v)\right)=C_{G}(v) \cap Q_{2} Q_{3}$ and $L_{2,3} \cong S L(2, q) \times S L(2, q) . \quad U_{0}^{\prime}=$ $Z\left(U_{0}\right), U_{\alpha} U_{\beta}=Z\left(C_{G}(v)\right), U_{0}=\left[U_{0}, L_{2,3}\right]$, and for $q>2 C_{G}(v)=C_{G}(v)^{\prime}=$ $O^{2}\left(C_{G}(v)\right)$.

(13.4) For $q>2$ there is a subgroup $H_{0}=H_{1} \times H_{2} \leq H$ such that $H_{1}$, $H_{2}$ are cyclic of order $q-1, H_{1}$ centralizes $U_{\alpha}$ and is fixed-point-free on $U_{\beta}$, and $\mathrm{H}_{2}$ centralizes $U_{\beta}$ and is fixed-point-free on $U_{\alpha}$. Also $q>2$ implies that $U_{\alpha}^{\sharp} U_{\beta}^{\sharp}$ is determined uniquely by the abstract structure of the group $C_{G}(v)$.

For the case $q>2$ we consider the centralizer of a certain subgroup of $H$.

(13.5) Let $q\rangle 2$ and set $W_{0}=H \cap\left\langle U_{ \pm r}\right\rangle, W_{1}=H \cap\left\langle U_{ \pm s}\right\rangle$. Then $C_{G}\left(W_{i}\right)$ $=W_{i} \times L_{i}$, when $L_{0}=\left\langle U_{ \pm \alpha_{2}}, U_{ \pm \alpha_{3}}, U_{ \pm \alpha_{4}}\right\rangle$ and $L_{1}=\left\langle U_{ \pm \alpha_{1}}, U_{ \pm \alpha_{2}}, U_{ \pm \alpha_{3}}\right\rangle$. Moreover $L_{0} \cong L_{1} \cong S p(6, q)$.

Proof. We deal with $W_{0}$, the proof for $W_{1}$ being similar. Let $P=$ $N_{G}\left(U_{r}\right)=Q\left(L_{0} \times W_{0}\right)$, where $Q=O_{2}(P)$. Then $W_{0}$ is fixed-point-free on $Q$. Similarly $W_{0}$ is fixed-point-free on $O_{2}\left(N_{G}\left(U_{-r}\right)\right)$. We conclude that $L_{0}=\left\langle U_{\gamma}: \gamma \in \Delta, U_{\gamma} \leq C\left(W_{0}\right)\right\rangle$. In particular $L_{0}$ is invariant under $C_{N}\left(W_{0}\right)$, where $N / H=W$, the Weyl group. The Bruhat decomposition gives $C_{G}\left(W_{0}\right)=C_{U}\left(W_{0}\right) C_{N}\left(W_{0}\right) C_{U}\left(W_{0}\right)=\left\langle C_{U}\left(W_{0}\right), C_{N}\left(W_{0}\right)\right\rangle . \quad$ Now $\quad N_{G}\left(L_{0}\right) \geq L_{0} \times$ $\left\langle U_{ \pm r}\right\rangle$ and $L_{0} \times\left\langle U_{ \pm r}\right\rangle=\left\langle U_{\delta}: \delta \in \Delta, U_{\delta} \leq N\left(L_{0}\right)\right\rangle$, so $C_{N}\left(W_{0}\right) \leq N\left(L_{0} \times\left\langle U_{ \pm r}\right\rangle\right)$ and it follows that $C_{N}\left(W_{0}\right) \leq L_{0} \times\left\langle U_{ \pm r}\right\rangle$. The result follows.

\section{Section 14. Centralizers of involutions in ${ }^{2} E_{6}(q)$.}

In this section let $G={ }^{2} E_{6}(q), q$ even. Then $G$ has Weyl group of type $F_{4}$ and roots will be labeled as in Section 13 and Table 1 . The action of $W$ on $\Delta$ is as in Section 12.

From (12.7) we have

(14.1) Each involution in ${ }^{2} E_{6}(q), q$ even, is conjugate to one of the fol- 
lowing

$$
\begin{aligned}
\text { i) } & t=U_{r}(1) \\
\text { ii) } & u=U_{s}(1) \\
\text { iii) } & v=U_{\alpha}(1) U_{\beta}(1) .
\end{aligned}
$$

We now find the centralizers of $t, u$, and $v$. For this we will use the results of Section 4 in [7] for information concerning the parabolic subgroups $P_{1}$ and $P_{4}$. For $P_{1}$ we have $Q_{1}$ special of order $q^{21}$ with $Z\left(Q_{1}\right)=U_{r}=U_{r_{24}}$ and $L_{1} \cong S U(6, q)$ acts irreducibly on $Q / Z\left(Q_{1}\right)$. In addition $Q_{1} L_{1}=C_{G}\left(U_{r}\right)=C_{G}(t), L_{1}=L_{1}^{\prime},\left[L_{1}, Q_{1}\right]=Q_{1}, C_{G}(t)=C_{G}(t)^{\prime}$, and $H_{1}=C_{H}\left(L_{1}\right)$ acts regularly on $U_{r}^{\#}$. For $P_{4}$ we have $Q_{4}=O_{2}\left(P_{4}\right)$ and $R_{4}=Z\left(Q_{4}\right)$ has order $q^{8}$. Moreover $L_{1} \cong S O^{-}(8, q)$ acts on $R_{4}$ preserving a non-degenerate quadratic form and in this action the isotropic 1-spaces are conjugates of $U_{\alpha}, \alpha$ long. We will use the following notation for $\alpha$ a short root:

$$
V_{\alpha}=\left\{U_{\alpha}(c): c \in F_{q}\right\} .
$$

Then $V_{\alpha}$ has index $q$ in $U_{\alpha}$.

Consider $u=U_{s}(1)$, an anisotropic vector in $R_{4}$. Consequently $C_{L_{4}}(u)$ $\cong S O(7, q)$. From (3.1)(iv) we see that $C_{U_{\alpha_{3}}}(u)=V_{\alpha_{3}}$. Moreover $s_{3} \in$ $\left\langle V_{\alpha_{3}}, V_{-\alpha_{3}}\right\rangle \cong S L(2, q)$.

Then $U_{r_{1}} U_{r_{15}} V_{r_{18}} U_{r_{2}} V_{r_{19}} U_{r_{18}} V_{r_{9}} U_{r_{3}} U_{r_{4}}$ has order $q^{7}$ and hence equals $U \cap C_{L_{4}}(u)$. The Bruhat decomposition can now be used to show that $\left\langle U_{\alpha_{1}}, U_{\alpha_{2}}, V_{\alpha_{3}}, s_{1}, s_{2}, s_{3}\right\rangle=C_{L_{4}}(u)$.

Suppose $P=P_{i}^{q}$ is parabolic and $C_{G}(u) \leq P$. Since $C_{G}(u)$ involves $S O(7, q), i=1$ or 4 . If $i=1$, then since $\left|Q_{4}\right|=q^{24}>q^{21}=\left|Q_{1}\right|, Q_{4} \not Q_{i}^{g}$ and $\left(C_{G}(u)\right)^{\prime} Q_{i}^{g} / Q_{i}^{g}$ is in a proper parabolic subgroup of $L_{4} \cong S U(6, q)$. But again there is no room for $S O(7, q)$. So $i=4$. The above arguments easily imply that $Q_{4}=Q_{4}^{g}$, so $g \in N\left(Q_{4}\right)=P_{4}$. Then 2.3 guarantees that $C_{G}(u) \leq P$ for some proper parabolic subgroup $P$, so together with the above we have $C_{G}(u)=C_{P_{4}}(u)=Q_{4}\left(K_{4} \times H_{1}\right)$.

Next we determine $C_{G}(v)$, where $v=U_{\alpha}(1) U_{\beta}(1)$. We first note that $(\alpha) s_{1}=\alpha=(\alpha) s_{4}$ and $(\beta) s_{1}=\beta=(\beta) s_{4}$. Also $U_{\alpha_{1}}$ centralizes $U_{\alpha} U_{\beta}$. However, $U_{\alpha_{4}} \cap C(v)=U_{\alpha_{4}} \cap C\left(U_{\alpha}(1)\right)=V_{\alpha_{4}}$ a subgroup of order $q$. Indeed if $\gamma \in \Delta$ and $\alpha+\gamma \in \Delta$, then $C(v) \cap U_{\gamma}=V_{\gamma}$ (see (3.1)(vi)). Then the Chevalley relations imply that $s_{4} \in\left\langle V_{ \pm \alpha_{4}}\right\rangle=\left\langle V_{\alpha_{4}}, s_{4}\right\rangle \cong S L(2, q)$. So $\left\langle U_{ \pm \alpha_{1}}\right\rangle$ $\times\left\langle V_{ \pm \alpha_{4}}\right\rangle=C_{L_{2,3}}(v)$.

Next use (3.1) and Table 1 to verify that 


$$
\begin{aligned}
& C_{U}\left(U_{\alpha}\right)=\left\langle U_{r}: r \neq \alpha_{3}, \alpha_{3}+\alpha_{4}, \alpha_{4}, \alpha_{2}+2 \alpha_{3}+\alpha_{4}, \alpha_{1}+\alpha_{2}+2 \alpha_{3}+\alpha_{4}\right\rangle \\
& C_{U}\left(U_{\beta}\right)=\left\langle U_{r}: r \neq \alpha_{2}, \alpha_{1}+\alpha_{2}\right\rangle .
\end{aligned}
$$

But also $v$ is centralized by $V_{\alpha_{4}}, V_{\alpha_{2}+2 \alpha_{3}+\alpha_{4}}$, and $V_{\alpha_{1}+\alpha_{2}+2 \alpha_{3}+\alpha_{4}}$. Consequently if we set $U_{0}=C(v) \cap Q_{2} Q_{3}=C(v) \cap O_{2}\left(P_{2,3}\right)$ we can use (3.1) to check that

$$
C_{U}(v)=U_{0}\left(U_{\alpha_{1}} \times V_{\alpha_{4}}\right)
$$

and

$$
U_{0}=\left\langle U_{r}: r \in \mathscr{L}_{2} \cup \mathscr{L}_{3}, r \neq r_{15}, r_{18}, r_{2}, r_{23}, r_{21}, r_{11}\right\rangle V_{r_{21}} V_{r_{11}} .
$$

In particular $\left|U: C_{U}(v)\right|=q^{9}$ and $\left|U_{0}\right|=q^{25}$. We first determine the structure of $U_{0}$ and the action of $\left\langle U_{ \pm \alpha_{1}}\right\rangle \times\left\langle V_{ \pm \alpha_{4}}\right\rangle$ on $U_{0}$. Using (3.1) and Table 1 we have $\left[U_{r_{14}}, U_{r_{9}}\right]=U_{r_{24}},\left[U_{r_{5}}, U_{r_{16}}\right]=U_{r_{7}}=U_{\beta},\left[U_{r_{5}}, U_{r_{19}}\right]=$ $U_{r_{14}} U_{r_{8}},\left[U_{r_{9}}, U_{r_{20}}\right]=U_{r_{12}}=U_{\alpha},\left[U_{r_{20}}, U_{r_{3}}\right]=U_{r_{13}} U_{r_{8}},\left[U_{r_{19}}, U_{r_{9}}\right]=U_{r_{4}}$, and $\left[U_{r_{10}}, U_{r_{20}}\right]=U_{r_{6}}$. It is easy to see that

$$
U_{0}^{\prime}=\left(U_{\alpha} U_{\beta}\right) \times\left(U_{r_{8}} U_{r_{24}}\right) \times\left(U_{r_{13}} U_{r_{14}}\right) \times\left(U_{r_{4}} U_{r_{8}}\right) \text {. }
$$

If we set $U_{1}=\left\langle U_{r}: r \in \mathscr{L}_{2} \cup \mathscr{L}_{3}, r \neq r_{15}, r_{18}, r_{2}, r_{23}, r_{21}, r_{11}\right\rangle$, then we also have

$$
U_{1}^{\prime}=U_{0}^{\prime},\left[U_{1}, U_{1}, U_{1}\right]=U_{r_{8}} U_{r_{24}}, \quad \text { and } \quad U_{1}^{\prime} \cap Z\left(U_{1}\right)=U_{\alpha} U_{\beta} U_{r_{8}} U_{r_{24}} .
$$

We then find that

$$
\left[U_{0}, U_{0}, U_{0}\right]=U_{r_{8}} U_{r_{24}} \text { and } U_{\beta} U_{r_{8}} U_{r_{24}} \leq Z\left(U_{0}\right) \cap U_{0}^{\prime} \text {. }
$$

It is easy to see that $\left\langle U_{ \pm \alpha_{1}}\right\rangle$ acts irreducibly on $U_{r_{8}} U_{r_{24}}$ and $\left\langle V_{ \pm \alpha_{4}}\right\rangle$ acts without fixed points on $U_{r_{18}} U_{r_{14}}$. Also $\left\langle V_{ \pm \alpha_{4}}\right\rangle$ acts irreducibly on $U_{0}^{\prime} / U_{\alpha} U_{\beta} U_{r_{8}} U_{r_{24}} U_{r_{13}} U_{r_{14}}$. From here and (3.1) one easily determines the action of $\left\langle U_{ \pm \alpha_{1}}\right\rangle \times\left\langle V_{ \pm \alpha_{4}}\right\rangle$ on $U_{0}^{\prime}$. Also it is easy to see that $\left[\left\langle U_{ \pm \alpha_{1}}\right\rangle \times\right.$ $\left.\left\langle V_{ \pm \alpha_{4}}\right\rangle, U_{0} / U_{0}^{\prime}\right]=U_{0} / U_{0}^{\prime}$ and all composition factors for $\left\langle U_{ \pm \alpha_{1}}\right\rangle \times\left\langle V_{ \pm \alpha_{4}}\right\rangle$ on $U_{0} / U_{0}^{\prime}$ are of order $q^{2}$ and isomorphic to the usual module for $S L(2, q)$ $\cong\left\langle U_{ \pm \alpha_{1}}\right\rangle \cong\left\langle V_{ \pm \alpha_{4}}\right\rangle$. In particular if $X=U_{0}\left(\left\langle U_{ \pm \alpha_{1}}\right\rangle \times\left\langle V_{ \pm \alpha_{4}}\right\rangle\right)$, then $X^{\prime}=$ $X$ if $q>2$ and $X^{\prime}=U_{0}\left(O_{3}\left(\left\langle U_{ \pm \alpha_{1}}\right\rangle \times\left\langle V_{ \pm \alpha_{4}}\right\rangle\right)\right)$ if $q=2$.

From (3.1)(vi) we have $V_{\alpha}=C_{U_{\alpha}}\left(V_{r_{11}}\right)$ and $V_{\alpha}=C_{U_{\alpha}}\left(V_{r_{21}}\right)$. Consequently $V_{\alpha} U_{\beta}=Z(X)$. Next we note that since some element of $W$ conjugates the pair $(\alpha, \beta)$ to $\left(\alpha_{4}, \alpha_{1}\right), H$ contains a subgroup $H_{0}=H_{1} \times H_{2}$ such that $H_{1} \cong H_{2}$ is cyclic of order $q-1, H_{1}$ is regular on $U_{\beta}^{\#}$ and centralizes $V_{\alpha}$, while $H_{2}$ is regular on $V_{\alpha}$ and centralizes $U_{\beta}$. Also we see that $\left|C_{H}\left(U_{\alpha} U_{\beta}\right)\right|=\left|C_{H}(v)\right|=(q-1)^{2}(q+1)$ so there is a subgroup 
$H_{\infty} \leq H$ such that $H_{\infty}$ is cyclic of order $q+1$ and $X H_{00}=C_{P_{2,3}}(v)$.

The next step is to determine the maximal parabolic subgroups of $G$ containing $X H_{\infty}$. Once we have this information we will be able to prove that $X H_{0}=C_{G}(v)$. The claim is that the only maximal parabolic subgroups of $G$ containing $X H_{0}$ are $P_{2}, P_{3}, P_{1}^{s_{1} s_{2}}$, and $P_{4}^{s_{4} s_{3}}$.

Suppose $C_{G}(v) \leq P_{1}^{g}$. As in the case of $F_{4}(q)$ we first assume that $U_{0} \cap O_{2}\left(P_{1}^{q}\right)^{\prime}=1$. Then $U_{0} \cap O_{2}\left(P_{1}^{q}\right)$ is abelian and the structure of $Q_{1}$ forces $\left|U_{0} \cap O_{2}\left(P_{1}^{q}\right)\right| \leq q^{10}$. Hence $\left.\left|U_{0} O_{2}\left(P_{1}^{q}\right) / O_{2}\left(P_{1}^{g}\right) \geq q^{15}=\right| L_{1}\right|_{2}$. As $X H_{\infty}$ involves $S L(2, q) \times S L(2, q)$ this is impossible. Consequently $U_{0} \cap O_{2}\left(P_{1}^{g}\right)^{\prime}$ $\neq 1$, and so $U_{0} \cap U_{r}^{g} \neq 1$. As before $X \leq O^{2^{\prime}}\left(P_{1}^{g}\right)=C\left(U_{r}^{g}\right)$ implies $U_{r}^{g} \cap U_{0}$ $\leq Z(X)=V_{\alpha} U_{\beta}$, and consequently $U_{r}^{g}=U_{\beta}$. Also $r=\beta^{s_{2} s_{1}}$, so $g \in P_{1} s_{1} s_{2}$ and $P_{1}^{g}=P_{1}^{s_{1} s_{2}}$.

Suppose that $X H_{\infty} \leq P_{4}^{g}$. As before we obtain a contradiction if $U_{0} \cap O_{2}\left(P_{4}^{g}\right)^{\prime}=U_{0} \cap\left(Q_{4}^{g}\right)^{\prime}=1$. Since $\left\langle U_{ \pm \alpha_{1}}\right\rangle$ acts irreducibly on $\left[U_{0}, U_{0}, U_{0}\right]$ $=U_{r_{8}} U_{r_{24}}$, either $U_{r_{8}} U_{r_{24}} \leq\left(Q_{4}^{g}\right)^{\prime}$ or $U_{r_{8}} U_{r_{24}} \cap\left(Q_{4}^{g}\right)^{\prime}=1$. If $U_{r_{8}} U_{r_{24}} \leq\left(Q_{4}^{g}\right)^{\prime}$, then $Q_{4}^{g} \leq C\left(U_{r_{8}} U_{r_{24}}\right)$. But $N\left(U_{r_{8}} U_{r_{24}}\right)=P_{2}$, so we have $Q_{4}^{g} \leq P_{2}$. In fact $Q_{4}^{g} \leq C\left(U_{r_{8}} U_{r_{24}}\right)$ implies that $Q_{4}^{g} \leq Q_{2} L_{12}$. Write $g=b w u$ where $b \in B$, $w \in W, u \in U$. Then $Q_{4}^{w} \leq Q_{2} L_{12}=Q_{2}\left\langle U_{ \pm \alpha_{3}}, U_{ \pm \alpha_{4}}\right\rangle$, and so there is an element $w_{1} \in\left\langle s_{3}, s_{4}\right\rangle$ such that $Q_{4}^{w w_{1}} \leq U$. But it is easily checked that for $w_{2} \in W,\left(\mathscr{L}_{4}\right) w_{2} \subset \Delta^{+}$implies $w_{2} \in\left\langle s_{1}, s_{2}, s_{3}\right\rangle$. Consequently $w \in P_{4}\left\langle s_{3}, s_{4}\right\rangle$. As $\left\langle U_{ \pm \alpha_{1}}\right\rangle \times\left\langle V_{ \pm \alpha_{4}}\right\rangle \leq P_{4}^{g}=P_{4}^{w u}$, we have $w \in P_{4} s_{4} s_{3}$. Using the Bruhat decomposition we may assume that $u \in U_{\alpha_{3}} U_{\alpha_{3}+\alpha_{4}}$ and from here we get $u=1$. Consequently $P_{4}^{g}=P_{4}^{s_{4} s_{3}}$ as claimed. So assume $U_{r_{8}} U_{r_{24}} \cap\left(Q_{4}^{g}\right)^{\prime}=1$. As $\left[U_{0}, U_{0}, U_{0}\right]=U_{r_{8}} U_{r_{24}}, U_{0}^{\prime} \cap\left(Q_{4}^{g}\right)^{\prime} \leq Z\left(U_{0}\right)$ and hence $U_{0}^{\prime} \cap\left(Q_{4}^{g}\right)^{\prime} \leq V_{\alpha} U_{\beta}$. As $\left|U_{0}\right|>\left|Q_{4}^{g}\right|, X / U_{0}$ is in a proper parabolic subgroup of $\left(P_{4} / Q_{4}\right)^{g}$ and $\left|U_{0} Q_{4}^{g} / Q_{4}^{g}\right| \leq q^{9}$. Hence $\left|U_{0} \cap Q_{4}^{g}\right| \geq q^{16}$. The commutator relations in (3.1) imply that for $g \in U_{0}-U_{0}^{\prime}$, there is an element $h \in U_{0}$ such that $[g, h] \leq$ $V_{\alpha} U_{\beta}$. Consequently $g \notin U_{0} \cap\left(Q_{4}^{g}\right)^{\prime}$ and $U_{0} \cap\left(Q_{4}^{g}\right)^{\prime} \leq V_{\alpha} U_{\beta}$. Thus $\left|U_{0} \cap Q_{4}^{g}\right|$ $\leq q^{18}$, and since $U_{0}\left(Q_{4}^{g}\right)^{\prime} \neq Q_{4}^{g},\left|U_{0} \cap Q_{4}^{g}\right|<q^{18}$ and $\left|U_{0} Q_{4}^{g} / Q_{4}^{g}\right|>q^{7}$. As $U_{0}\left(Q_{4}^{g}\right)^{\prime} \neq Q_{4}^{g}, V_{\alpha} U_{\beta} \cap\left(Q_{4}^{g}\right)^{\prime} \neq 1$ and so $X$ fixes a 1 -space of the orthogonal space $R_{4}^{g}$. Since $X Q_{4}^{g} / Q_{4}^{g}$ has Sylow 2-subgroups of order at least $2 q^{9}$ this space must be singular. Then consideration of the parabolic subgroups of $\left(P_{4} / Q_{4}\right)^{g}$ contradicts the fact that $X Q_{4}^{g} / Q_{4}^{g}$ involves $S L(2, q) \times$ $S L(2, q)$.

If $X H_{\infty} \leq P_{2}^{g}$, then we argue as in the case of $F_{4}(q)$ to get $P_{2}^{g}=P_{2}$. So we are left with the case $X H_{\infty} \leq P_{3}^{g}$. Here the situation is a little different than in $F_{4}(q)$ as the parabolic subgroups are not permuted by 
a graph isomorphism centralizing the involution. First we note that $U_{0} \not Q_{3}^{g}$. Indeed $\left|U_{0} / U_{0}^{\prime}\right|=q^{14}$ while $Q_{3}^{\prime}=\left\langle U_{r}: r \in \mathscr{L}_{i}^{3} \cup \mathscr{S}_{i}^{3}\right.$ for $\left.i \geq 2\right\rangle$ and $\left|Q_{3} / Q_{3}^{\prime}\right|=q^{12}$. So $X Q_{3}^{g} / Q_{3}^{g}$ is contained in a proper parabolic subgroup of $O^{2^{\prime}}\left(P_{3}^{g} / Q_{3}^{g}\right)$. As $X$ involves $S L(2, q) \times S L(2, q)$ this parabolic subgroup has the form $P_{3}^{g} \cap P_{i}^{g x}$ for some $x \in P_{3}^{g}$ and $i=1$ or 2 . At this stage we can use the same argument presented for $F_{4}(q)$. We have now determined the necessary information on parabolic subgroups.

At this point we can prove that $X H_{\infty}=C_{G}(v)$. We know by (2.3) that $C_{G}(v) \leq P$ for some proper parabolic subgroup $P$. Consequently $X H_{0} \leq C_{G}(v) \leq P$ and $P=P_{2}, P_{3}, P_{1}^{s_{1} s_{2}}$, or $P_{4}^{s_{4} s_{3}}$. If $C_{G}(v) \leq P_{2}$, then $g \in C_{G}(v)$ implies $g=b w u$ where $w \in\left\langle s_{1}\right\rangle \times\left\langle s_{3}, s_{4}\right\rangle$ and it is easy to check that $w \in\left\langle s_{1}\right\rangle \times\left\langle s_{4}\right\rangle$. Then $C_{G}(v) \leq P_{2} \cap P_{4}$ and $X H_{\infty}=C_{P_{2} \cap P_{3}}(v)$. Similarly we are done if $C_{G}(v) \leq P_{3}$. If $C_{G}(v) \leq P_{1}^{s_{1} s_{2}}=N_{G}\left(U_{r}^{s_{1} s_{2}}\right)=N_{G}\left(U_{\beta}\right)$, then it will follow that $C_{G}(v) \cap Q_{1}^{s_{1} s_{2}} L_{1}^{s_{1} s_{2}} \leq C_{G}\left(U_{\beta}\right) \cap C_{G}(v) \leq C_{G}\left(U_{\alpha}(1)\right) \leq P_{4}^{s_{4} s_{3}}$. Then $C_{G}(v) \leq P_{1}^{s_{1} s_{2}} \cap P_{4}^{s_{4} s_{3}}$ and again it is easy to check that $C_{G}(v) \leq P_{2} \cap P_{3}$ and hence $C_{G}(v)=X H_{\infty}$. Say $C_{G}(v) \leq P_{4}^{s_{4} s_{3}}$. Then we are done if $O_{2}\left(C_{G}(v)\right)$ $\measuredangle Q_{4}^{s_{4} s_{3}}$ by a previous case. So $O_{2}\left(C_{G}(v)=C_{G}(v) \cap O_{2}\left(P_{4}^{s_{4} s_{3}}\right)\right.$ and this is easily seen to be $U_{0} \cap O_{2}\left(P_{4}^{s_{4} s_{3}}\right)$. Then $\left(U_{0} \cap O_{2}\left(P_{4}^{s_{4} s_{3}}\right)\right)^{\prime}$ generates a subspace of $R_{4}^{s_{4} s_{3}}$ stabilized by $C_{G}(v)$. But $\left(U_{0} \cap O_{2}\left(P_{4}^{s_{4} s_{3}}\right)\right)^{\prime}=U_{r_{8}} U_{r_{24}}$ is an isotropic 2-space of $R_{4}^{s_{4} s_{3}}$ and hence $C_{G}(v) \leq P_{4}^{s_{4} s_{3}} \cap P_{2}$. From here we get $C_{G}(v) \leq$ $Q_{4}^{s_{4} s_{3}}\left\langle U_{ \pm \alpha_{1}}, U_{ \pm \alpha_{4}}\right\rangle H$ and checking we have $C_{G}(v)=X H_{\infty}$.

As in the case of $F_{4}(q)$ we show that $V_{\alpha}^{\sharp} U_{\beta}^{\#}$ is determined uniquely by the abstract structure of $C_{G}(v)$. Summarizing

(14.2) The maximal parabolic subgroups of $G$ containing the centralizers of the involutions in (14.1) are as follows:
i) $C_{G}(t) \leq P_{1}$
ii) $C_{G}(u) \leq P_{4}$
iii) $C_{G}(v) \leq P_{2}, P_{3}, P_{1}^{s_{1} s_{2}}, P_{4}^{s_{4} s_{3}}$.

In particular $G$ has 3 classes of involutions.

(14.3) With notation as in (14.1) we have

i) $C_{G}(t)=\left(C_{G}(t)\right)^{\prime}=O^{2^{\prime}}\left(P_{1}\right), Z\left(C_{G}(t)\right)=Q_{1}^{\prime}=U_{r}$

ii) $C_{G}(u)=Q_{4}\left(K_{4} \times H_{1}\right)$. Here $H_{1}=C_{G}\left(Q_{4}^{\prime}\right)$ is cyclic of order $q+1$, $K_{4}=\left\langle U_{\alpha_{1}}, U_{\alpha_{2}}, V_{\alpha_{3}}, s_{1}, s_{2}, s_{3}\right\rangle \cong S O(7, q)$, where $V_{\alpha_{3}}=C(u) \cap U_{\alpha_{3}}$ and $\left|V_{\alpha_{3}}\right|$ =q. Also $\left[K_{4}, Q_{4}\right]=Q_{4}, C_{G}(u)^{\prime}=C_{G}(u)$, and $Z\left(Q_{4} L_{4}\right)=V_{s}=Z\left(C_{G}(u)\right)=$ $\left\{U_{s}(c): c \in F_{q}\right\}$.

iii) $C_{G}(v)=U_{0}\left(\left\langle U_{\alpha_{1}}, s_{1}\right\rangle \times\left\langle V_{\alpha_{4}}, s_{4}\right\rangle H_{\infty}\right.$, where $U_{0}=\left\langle U_{r}: r \in \mathscr{L}_{2} \cup \mathscr{L}_{3}\right.$, 
$r \neq r_{2}, r_{11}, r_{15}, r_{18}, r_{21}, r_{23}>V_{r_{11}} V_{r_{21}} . \quad V_{r}=U_{r} \cap C(v)$ is elementary of order $q$, and $H_{\infty}$ is cyclic of order $q+1$. Moreover $\left[U_{0}, U_{0}, U_{0}\right]=U_{r_{8}} U_{r_{24}}$ and $Z\left(C_{G}(v)\right)=V_{\alpha} U_{\beta}$, where $V_{\alpha}=C_{U_{\alpha}}\left(V_{r_{11}}\right)$. Also $O^{2^{\prime}}\left(C_{G}(v) / U_{0}\right) \cong S L(2, q) \times$ $S L(2, q)$ and $\left[U_{0}, C_{G}(v)\right]=U_{0}$. If $\left.q\right\rangle 2$ then $C_{G}(v)^{\prime}=U_{0}\left(\left\langle U_{\alpha_{1}}, s_{1}\right\rangle \times\left\langle V_{\alpha_{4}}, s_{4}\right\rangle\right)$. If $q=2$, then $C_{G}(v)^{\prime}=U_{0}\left(O_{3}\left(\left\langle U_{\alpha_{1}}, s_{1}\right\rangle \times\left\langle V_{\alpha_{4}}, s_{4}\right\rangle\right)\right) \unlhd C_{G}(v)$.

(14.4) For $q>2$ there is a subgroup $H_{0}=H_{1} \times H_{2} \leq H$ such that $H_{1}$, $H_{2}$ are cyclic of order $q-1, H_{1}$ centralizes $V_{\alpha}$ and is fixed-point-free on $U_{\beta}$, while $H_{2}$ centralizes $U_{\beta}$ and is fixed-point-free on $V_{\alpha}$. The set $V_{\alpha}^{\#} U_{\beta}^{\#}$ is uniquely determined by the abstract structure of $C_{G}(v)$.

We complete this section by finding the centralizer of a certain subgroup of $H$.

(14.5) Let $q>2$ and set $W_{0}=H \cap\left\langle U_{ \pm r}\right\rangle, \hat{W}_{1}=H \cap\left\langle U_{ \pm s}\right\rangle$, and $W_{1}=$ $\left(\hat{W}_{1}\right)^{q+1}$. Then $C_{G}\left(W_{0}\right)=W_{0} L_{0}$ and $C_{G}\left(W_{1}\right)=\hat{W}_{1} L_{1}$, when $L_{0}=\left\langle U_{ \pm \alpha_{2}}, U_{ \pm \alpha_{3}}\right.$, $\left.U_{ \pm \alpha_{4}}\right\rangle$ and $L_{1}=\left\langle U_{ \pm \alpha_{1}}, U_{ \pm \alpha_{2}}, U_{ \pm \alpha_{3}}\right\rangle$. Moreover $L_{0} \cong S U(6, q)$ and $L_{1} \cong$ $\mathrm{SO}^{-}(8, q)$.

Proof. The proof is similar to the proof of (13.5).

\section{Section 15. Centralizers of involutions in $E_{6}(q), q$ even.}

Let $G=E_{6}(q)$ with $q=2^{a}$. Then $|H|=(q-1)^{6}(3, q-1)^{-1}, W=$ $\left\langle s_{1}, \cdots, s_{6}\right\rangle$ and the action of $W$ on $\Delta$ is determined by

$$
\begin{aligned}
& \left(\alpha_{3}\right) s_{1}=\left(\alpha_{1}\right) s_{3}=\alpha_{1}+\alpha_{3} \\
& \left(\alpha_{4}\right) s_{2}=\left(\alpha_{2}\right) s_{4}=\alpha_{2}+\alpha_{4} \\
& \left(\alpha_{j}\right) s_{i}=\alpha_{i}+\alpha_{j} \text { if }|i-j|=1 \text { and }\{i, j\} \neq\{1,2\},\{2,3\} \\
& \left(\alpha_{i}\right) s_{i}=-\alpha_{i},
\end{aligned}
$$

with $\left(\alpha_{j}\right) s_{i}=\alpha_{j}$ for all other pairs $(i, j)$. We remark that in computations with commutators (3.1)(ii) is the only relevant commutator, making such calculations particularly easy.

We use the notion of Table 2 , distinguishing the roots $r, \alpha, \beta, \gamma, \delta, \varepsilon$ in $\Delta^{+}$. By (12.8) we have

(15.1) Each involution in $E_{6}(q)$, q even, is conjugate to one of the following
i) $x=U_{r}(1)$
ii) $y=U_{\alpha}(1) U_{\beta}(1)$
iii) $z=U_{r}(1) U_{\delta}(1) U_{\diamond}(1)$. 
We will see in (15.4) that no two of $x, y, z$ are conjugate. This will be clear from the structure of the centralizers of these involutions.

Let $x=U_{r}(1)$. We use the results in Section 4 of [7] to see that $C_{G}(x)=O^{2}\left(P_{2}\right)=P_{2}^{\prime}=Q_{2} L_{2}$. Also $P_{2}=Q_{2} L_{2} W_{0}$, where $\left[L_{2}, W_{0}\right]=1, W_{0}$ is cyclic of order $q-1$, and $W_{0} \cap L_{2}=Z\left(L_{2}\right) . \quad C_{G}\left(W_{0}\right)=W_{0} L_{2}$.

The method for finding the other centralizers is as follows, and will be used repeatedly in Section 16 and Section 17. Say $t=U_{\beta_{1}}(1) \cdots U_{\beta_{k}}(1)$ is one of the involutions in (15.1), (16.1), or (17.1) with $k>1$. We will exhibit a certain parabolic subgroup $P \geq B$ with Levi factor $L$ such that $C_{L}(t) \geq L_{0}$, where $L_{0}$ is generally obtained from $L$ as the fixed points of a graph automorphism. Usually $L_{0}$ will be maximal in $L$ and since $L \not \subset C(t)$, we obtain $L_{0}=C_{L}(t)$. Then we find $C_{0_{2}(P)}(t), C_{U}(t)$, and hence $C_{P}(t)$.

By (2.3) we know that $C_{G}(t)$ is contained in a proper parabolic subgroup of $G$. We find all parabolic subgroups of $G$ containing $C_{P}(t)$. There are very few of these and we are able to conclude that $C_{G}(t)=$ $C_{P}(t)$.

We obtained $L_{0}$ as follows. From the action of $W$ on the set of root groups we know that $C_{W}(t)$ is just the stabilizer in $W$ of $\left\{t_{1}, \cdots, t_{k}\right\}$. This can be found by first finding $C_{W}\left(t_{1}\right) \cap \ldots \cap C_{W}\left(t_{k}\right)$ and then studying the induced group. The roots $t_{1}, \cdots, t_{k}$ have been chosen so that $C_{W}(t)$ is clearly the set of fixed points of a parabolic subgroup of $W$ under a graph automorphism. From here $P, L, L_{0}$ are obvious.

Consider $C_{G}(y)$ where $y=U_{\alpha}(1) U_{\beta}(1)$. The root groups $U_{s} \leq U$ not centralizing $U_{\alpha}(1)$ and $U_{\beta}(1)$ are as follows:

$$
\begin{array}{lll}
U_{\alpha}(1) & \left\{s=r_{34}, r_{32}, r_{20}\right\} & \left\{s+\alpha=r_{14}, r_{15}, r_{16}\right\} \\
U_{\beta}(1) & \left\{s=r_{27}, r_{28}, r_{19}\right\} & \left\{s+\beta=r_{14}, r_{15}, r_{16}\right\} .
\end{array}
$$

It is then straightforward to check that

$$
\begin{aligned}
& C_{U}\left(U_{\alpha} U_{\beta}\right)=\left\langle U_{r}: r \in \Delta^{+}, r \neq r_{19}, r_{20}, r_{27}, r_{28}, r_{32}, r_{34}\right\rangle \\
& C_{U}(y)=\left\langle C_{U}\left(U_{\alpha} U_{\beta}\right), U_{r_{34}}(c) U_{r_{27}}(c), U_{r_{32}}(c) U_{r_{28}}(c), U_{r_{20}}(c) U_{r_{19}}(c): c \in \boldsymbol{F}_{q}\right\rangle .
\end{aligned}
$$

In particular $\left|C_{U}\left(U_{\alpha} U_{\beta}\right)\right|=q^{30},\left|C_{U}(y)\right|=q^{33}$, and $\left|U: C_{U}(y)\right|=q^{3}$.

Let $L_{0}=\left\langle U_{\alpha_{3}}(c) U_{\alpha_{5}}(c), U_{\alpha_{4}}, U_{\alpha_{2}}, s_{3} s_{5}, s_{4}, s_{2}: c \in F_{q}\right\rangle$. Then $L_{0} \leq L$, where $L$ is the Levi factor of $P_{1,6}$. Moreover it is straightforward to check that $L_{0} \leq C(y)$ and $L \not C(y)$. Now $L \cong D_{4}(q)$ and $L_{0}$ is obtained as the fixed group of a graph automorphism of $L$. It follows that $L_{0} \cong B_{3}(q)$. 
Let $w=s_{1} s_{6} s_{3} s_{5} s_{4} s_{2} s_{3} s_{5} s_{4} s_{3} s_{5}$. Then $\left(U_{\alpha} U_{\beta}\right)^{w}=U_{\alpha_{1}} \times U_{\alpha_{6}} \leq\left\langle U_{ \pm \alpha_{1}}\right\rangle \times$ $\left\langle U_{ \pm \alpha_{6}}\right\rangle$, so $H$ is transitive on $U_{\alpha}^{\#} U_{\beta}^{\#}$. Setting $H_{1}=C_{H}(y)$ we have $\left|H_{1}\right|=$ $(q-1)^{4}(3, q-1)^{-1}$.

Next we set $U_{0}=Q_{1} Q_{6}$ and $H_{0}=C_{H_{1}}(L)$. Then $L_{0} \times H_{0}$ normalizes $U_{0}$ and we set $X=U_{0}\left(L_{0} \times H_{0}\right)$. The first observation is that $U_{0} L_{0}=$ $\left\langle C_{U}(y), C_{W}(y)\right\rangle$. Consider the action of $L_{0}$ on $Q_{1} Q_{6}$. Since $Q_{1}, Q_{6}$ are elementary it is easy to check that $Z\left(U_{0}\right)=Q_{1} \cap Q_{6}=U_{0}^{\prime}$ and that $\left|Z\left(U_{0}\right)\right|$ $=q^{8}$. Using Table 2 and (3.1) we also check that $\left[L_{0}, U_{0}\right]=U_{0}$. As $U_{\alpha}^{\sharp} U_{\beta}^{\sharp}$ is fused by $H$ we see that $\left(U_{\alpha} U_{\beta}\right)^{\#}$ contains $(q-1)^{2}$ conjugates of $y$ and $2(q-1)$ conjugates of $x$. Set $P=\left\langle U_{\alpha}(c) U_{\beta}(c): c \in \boldsymbol{F}_{q}\right\rangle$. Then choosing $h \in H$ such that $h$ centralizes $U_{\beta}$ but not $U_{\alpha}$ we have $P P^{h}=U_{\alpha} U_{\beta}$. Also $P=Z(X)$.

We will show that $X=C_{G}(y)$ and that $P_{1}, P_{6}$ are the only parabolic subgroups of $G$ containing $X$. Suppose that $\hat{P}=P_{i}^{g} \geq X$. Since $X / O_{2}(X)$ involves $S p(6, q)$ we must have $i=1$ or 6 . Then $O_{2}(\hat{P})$ is abelian and so $O_{2}(X) \not O_{2}(\hat{P})$ and $X$ is contained in a proper parabolic subgroup of $P$. This parabolic subgroup has the form $P_{i}^{g} \cap P_{j}^{g k}$, where $j \in\{1,6\}-$ $\{i\}$ and $k \in P_{i}^{g}$. This implies that $X \leq\left(P_{1} \cap P_{6}\right)^{p_{i} g}$, for some $p_{i} \in P_{i}$. On the other hand at this point we must have $O_{2}(X) \leq O_{2}\left(\left(P_{1} \cap P_{6}\right)^{p_{i} g}\right)$ and by orders, equality holds. Since $P_{1} \cap P_{6}=N_{G}\left(Q_{1} Q_{6}\right), p_{i} g \in P_{1} \cap P_{6}$ and $P_{i}^{g}=P_{i}$.

To see that $X=C_{G}(y)$ we note that $C_{G}(y)$ is contained in a parabolic subgroup of $G$ and the graph automorphism of $G$ centralizes $y$. Consequently $C_{G}(y) \leq P_{1} \cap P_{6}$. Now $L_{1,6}$ operates on $Z\left(Q_{1} Q_{6}\right)=\left(Q_{1} Q_{6}\right)^{\prime}$ and using Table 2 it is easy to see that $L_{1,6}$ preserves a non-degenerate quadratic form on $Z\left(Q_{1} Q_{6}\right)$ in which $Z\left(Q_{1} Q_{6}\right)$ is the orthogonal direct sum of hyperbolic planes $U_{\alpha} U_{\beta}, U_{r_{10}} U_{r_{14}}, U_{r_{7}} U_{r_{15}}$, and $U_{r_{8}} U_{r_{18}}$. In this action the elements of $U_{s}^{\#}$ for $s \in \Delta^{+}$are singular vectors, and $y$ is an anisotropic vector. So $C_{L_{1,6}}(y) \cong S O(7, q) \cong S p(6, q) \cong L_{0}$ and it follows that $X=C_{G}(y)$.

Now we consider $C_{G}(z), z=U_{\gamma}(1) U_{\delta}(1) U_{\varepsilon}(1)$. We proceed as before by first finding $C_{U}(z)$ and $C_{W}(z)$. The root groups $U_{s}$ for $s \in \Delta^{+}$not centralized by $U_{r}, U_{\delta}$, and $U_{\varepsilon}$ are as follows:

$$
\begin{array}{lll}
U_{r} & \left\{s=r_{1}, r_{2}, r_{3}, r_{4}\right\} & \left\{s+\gamma=r_{13}, r_{14}, r_{15}, r_{16}\right\} \\
U_{\delta} & \left\{s+r_{27}, r_{34}, r_{29}, r_{22}\right\} & \left\{s+\delta=r_{12}, r_{13}, r_{15}, r_{16}\right\} \\
U_{c} & \left\{s=r_{36}, r_{35}, r_{33}, r_{21}\right\} & \left\{s+\varepsilon=r_{12}, r_{14}, r_{15}, r_{16}\right\} .
\end{array}
$$


It is then easy to check that $C_{U}\left(U_{7} U_{\delta} U_{\mathrm{s}}\right)=\left\langle U_{s}: s \in \Delta^{+}\right.$and $s \neq r_{1}, r_{2}, r_{3}, r_{4}$, $\left.r_{21}, r_{22}, r_{27}, r_{29}, r_{33}, r_{34}, r_{35}, r_{36}\right\rangle$. Also it is easily seen that $z$ is centralized by each of $U_{\varphi}(c) U_{\psi}(c)$ for $(\varphi, \psi) \in C=\left\{\left(r_{1}, r_{34}\right),\left(r_{27}, r_{36}\right),\left(r_{2}, r_{35}\right),\left(r_{3}, r_{29}\right),\left(r_{29}, r_{33}\right)\right.$, $\left.\left(r_{4}, r_{22}\right),\left(r_{4}, r_{21}\right)\right\}$. We then have $C_{U}(z)=\left\langle C_{U}\left(U_{r} U_{\delta} U_{\varepsilon}\right), U_{\varphi}(c) U_{\psi}(c): c \in \boldsymbol{F}_{q}\right.$, $(\varphi, \psi) \in C\rangle$. In particular $\left|C_{U}(z)\right|=q^{31}$.

Let $L_{0}=\left\langle U_{ \pm \alpha_{2}}, s_{1} s_{5}, s_{3} s_{6}, U_{\alpha_{1}}(c) U_{\alpha_{5}}(c), U_{\alpha_{3}}(c) U_{\alpha_{6}}(c): c \in F_{q}\right\rangle$. Then $L_{0} \leq L$, where $L$ is the Levi factor of $P_{4}$, and it is easy to see that $L_{0}=C_{L}(z)$.

$$
\begin{aligned}
L_{0} & =\left\langle U_{ \pm \alpha_{2}}, s_{1} s_{5}, s_{3} s_{6}, U_{\alpha_{1}}(c) U_{\alpha_{5}}(c), U_{\alpha_{3}}(c) U_{\alpha_{6}}(c): c \in F_{q}\right\rangle \\
& \cong S L(2, q) \times S L(3, q) .
\end{aligned}
$$

Now we set

$$
\begin{aligned}
U_{0}= & \left\langle C_{Q_{4}}\left(U_{r} U_{\delta} U_{s}\right), U_{r_{3}}(c) U_{r_{29}}(c), U_{r_{29}}(c) U_{r_{33}}(c), U_{r_{4}}(c) U_{r_{22}}(c),\right. \\
& \left.U_{r_{4}}(c) U_{r_{21}}(c): c \in F_{q}\right\rangle \\
X= & U_{0} L_{0}=C_{P_{4}}(z)
\end{aligned}
$$

Next consider the structure of $U_{0}$ and the action of $L_{0}$ on $U_{0}$. We have $\left|U_{0}\right|=q^{27}$ and $X \leq P_{4}$. Checking Table 2 and (3.1) we easily see that $U_{0}^{\prime}=P_{4}^{\prime}=Q_{2}^{4} Q_{3}^{4}$, where $Q_{2}^{4}=\left\langle U_{s}: s \in \mathscr{L}_{2}^{4}\right\rangle$ and $Q_{3}^{4}=U_{r_{15}} \times U_{r_{18}}$. Also we check that $\left[U_{0}, U_{0}, U_{0}\right]=Q_{3}^{4} \leq Z\left(U_{0}\right)$ and that $\left\langle U_{ \pm \alpha_{2}}\right\rangle$ acts irreducibly on $\left[U_{0}, U_{0}, U_{0}\right]$. Next we can use Table 2 and (3.1) to verify that $\left[L_{0}, U_{0} / U_{0}^{\prime}\right]=U_{0} / U_{0}^{\prime}$, so that $\left[L_{0}, U_{0}\right]=U_{0}$. In doing so it is useful to note that modulo $U_{0}^{\prime}$ we have

$$
\begin{aligned}
& \left\langle U_{r_{3}}(c) U_{r_{29}}(c), U_{r_{29}}(c) U_{r_{33}}(c), U_{r_{4}}(c) U_{r_{22}}(c), U_{r_{4}}(c) U_{r_{21}}(c): c \in \boldsymbol{F}_{q}\right\rangle \\
& \quad=\left\langle U_{r_{3}}(c) U_{r_{29}}(c), U_{r_{4}}(c) U_{r_{22}}(c): c \in F_{q}\right\rangle \times\left\langle U_{r_{3}}(c) U_{r_{33}}(c), U_{r_{4}}(c) U_{r_{21}}(c): c \in \boldsymbol{F}_{q}\right\rangle
\end{aligned}
$$

and that $\left\langle U_{ \pm \alpha_{2}}\right\rangle$ acts on both factors as on the natural module for $\left\langle U_{ \pm \alpha_{2}}\right\rangle \cong S L(2, q)$.

Say $q>2$. For $w=s_{4} s_{3} s_{5} s_{2} s_{4} s_{3} s_{5} s_{1} s_{6} s_{2} s_{3} s_{5} s_{4}$, we have $\{\gamma, \delta, \varepsilon\}^{w}=\left\{\alpha_{2}, \alpha_{3}, \alpha_{5}\right\}$, so $\left(U_{\gamma} U_{\delta} U_{\varepsilon}\right)^{w}=U_{\alpha_{2}} \times U_{\alpha_{3}} \times U_{\alpha_{5}} \leq\left\langle U_{ \pm \alpha_{2}}\right\rangle \times\left\langle U_{ \pm \alpha_{3}}\right\rangle \times\left\langle U_{ \pm \alpha_{5}}\right\rangle$. Therefore there is a subgroup of $H$ of the form $H_{r} \times H_{\delta} \times H_{c}$ such that $H_{r}$ is regular on $U_{\gamma}^{\#}$ and centralizes $U_{\delta} U_{\varepsilon}$, and $H_{\delta}$ is regular on $U_{\delta}^{\#}$ and centralizes $U_{r} U_{\varepsilon}$, and $H_{\varepsilon}$ is regular on $U_{\varepsilon}^{\#}$ and centralizes $U_{r} U_{\delta}$. Let $P=$ $\left\langle U_{r}(c) U_{\delta}(c) U_{\theta}(c): c \in \boldsymbol{F}_{q}\right\rangle$. We check that $P=Z(X)$. Let $h \in H_{r}^{\sharp}$. Then $P P^{h}=\left\langle U_{r}(c) U_{\delta}(c) U_{\epsilon}(c), U_{r}(d c) U_{\delta}(c) U_{s}(c): c \in F_{q}\right\rangle$ for some fixed $1 \neq d \in \boldsymbol{F}_{q}^{\#}$. It follows that $P P^{h}=U_{r} \times P$ and that $P P^{h}$ contains $q-1$ conjugates of $x, q-1$ conjugates of $y$, and $(q-1)^{2}$ conjugates of $z$.

We must still show that $X=C_{G}(z)$ and find the parabolic subgroups 
of $G$ that contain $X$. Suppose that $X \leq P_{i}^{q}$. We note that for $i \neq 4$, $\mathscr{L}_{3}^{i}=\emptyset$ and consequently $Q_{i}$ has class at most 2 . Therefore $i \neq 4$ implies that $U_{0} \leq Q_{i}^{g}$. First assume that $i=4$. If $U_{0} \leq Q_{4}^{g}$, then $\left[U_{0}, U_{0}, U_{0}\right]=$ $\left[Q_{4}^{g}, Q_{4}^{g}, Q_{4}^{g}\right]$ and $P_{4}=N_{G}\left(\left[U_{0}, U_{0}, U_{0}\right]\right)=P_{4}^{g}$. Say $U_{0} \not Q_{4}^{g}$. Then $X Q_{4}^{g} / Q_{4}^{g}$ is contained in a parabolic subgroup of $P_{4}^{g} / Q_{4}^{g}$. Say $X Q_{4}^{g} / Q_{4}^{g}$ is contained in $P_{3}^{g p}$ for $p \in P_{4}^{g}$. Since $U_{0}=\left[U_{0}, L_{0}\right], U_{0}$ is contained in a conjugate $Q^{\ell}$ of $Q=Q_{3} Q_{4}$. Using Table 2 and (3.1) we check that $[Q, Q, Q]=Q_{2}^{4} Q_{3}^{4}$ and $[Q, Q, Q, Q]=Q_{3}^{4}$. Now $X / U_{0}$ involves $S L(3, q)$ and consequently $X$ covers the conjugate of $L_{1,2,3,4}=\left\langle U_{ \pm \alpha_{5}}, U_{ \pm \alpha_{6}}\right\rangle$ in $P_{4}^{g} \cap P_{3}^{g p} / O_{2}\left(P_{4}^{g} \cap P_{3}^{g p}\right)$. However it is easy to see that this group acts without fixed points on $\left[Q^{\ell}, Q^{\ell}, Q^{\ell}\right] /\left[Q^{\ell}, Q^{\ell}, Q^{\ell}, Q^{\ell}\right]$, while $\left[U_{0}, U_{0}, U_{0}\right]$ is centralized by the corresponding factor isomorphic to $S L(3, q)$. It follows that $\left[U_{0}, U_{0}, U_{0}\right] \leq$ $\left[Q^{\ell}, Q^{\ell}, Q^{\ell}, Q^{\ell}\right]$ and by orders these must be equal. Then $P_{4}=N_{G}\left(Q_{3}^{4}\right)=$ $N_{G}\left(\left[Q^{\ell}, Q^{\ell}, Q^{\ell}, Q^{\ell}\right]\right)=P_{4}^{g}$. But then $g \in P_{4}$ and $U_{0} \leq Q_{4}^{g}$, a contradiction. By symmetry we have $X Q_{4}^{g} / Q_{4}^{g}$ not contained in $P_{5}^{g p}$ for $p \in P$. Also this argument works to eliminate the cases of $X \leq P_{4}^{g} \cap P_{1}^{g p}$ or $P_{4}^{g} \cap P_{6}^{g p}$. So we are left with the case of $X \leq P_{4}^{g} \cap P_{2}^{g p}$. As the other parabolics have been eliminated we must have $U_{0} \leq O_{2}\left(P_{4}^{q} \cap P_{2}^{g p}\right)$ but $U_{0} \leq O_{2}\left(P_{4}^{g}\right)$. Since $P_{4} / O_{2}\left(P_{4}\right) \cong\left\langle U_{ \pm \alpha_{2}}\right\rangle \times S L(3, q) \times S L(3, q)$, we must have $O^{2^{\prime}}(X)<X$, whereas we have already seen that $O^{2}(X)=X$. So this is also a contradiction.

We now assume $X \leq P_{i}^{q} \cap P_{j}^{g p}=\hat{P}$ for $i \neq j$ and $i, j \neq 4$. Say $i=1$, $j=2$. Then $\left|Q_{1,2}\right|=q^{26}$ implies that $U_{0} \not O_{2}\left(P_{i}^{q} \cap P_{j}^{q p}\right)$ and $X$ is in a proper parabolic subgroup $\bar{P}$ of $P_{i}^{q} \cap P_{j}^{g p}$. By the above $\bar{P} \sim P_{1,2,5}$ and $X$ covers $O^{2^{\prime}}\left(\bar{P} / O_{2}(\bar{P})\right)$. Then $U_{0} \leq O_{2}(\bar{P})$ and $z \in U_{0}^{\prime} \leq O_{2}(\bar{P})^{\prime}$. Let $L_{00} \leq$ $L_{0}=S L(2, q) \times S L(3, q)$ be a direct sum of Singer cycles. Then checking the action of $L_{00}$ on $K=O_{2}(\bar{P})^{\prime}$ we have $C_{K}\left(L_{00}\right) \sim U_{r_{16}} U_{r_{26}}$. But $\left(U_{r_{18}} U_{r_{26}}\right)^{\#} \subseteq x^{G}$ and $P_{2}$ is the unique parabolic subgroup of $G$ containing $C_{G}(x)$. This is a contradiction. Similarly $(i, j) \neq(1,3),(1,6)$.

Next assume that $(i, j)=(1,5)$. By the above $U_{0} \leq O_{2}(\hat{P}) \sim Q_{1} Q_{5}$. From Table 2 and (3.1) we have $\left[Q_{1} Q_{5}, Q_{1} Q_{5}, Q_{1} Q_{5}\right]$ of order $q^{4}$ and centralized by $\left\langle U_{ \pm \alpha_{8}}\right\rangle$. Now $O^{2^{\prime}}\left(\hat{P} / O_{2}(\hat{P})\right) \cong S L(4, q) \times S L(2, q)$, where the factor of $S L(2, q)$ centralizes $\left[U_{0}, U_{0}, U_{0}\right]$. As $L_{0} \cong S L(3, q) \times S L(2, q)$ we have $\left\langle U_{ \pm \alpha_{6}}\right\rangle \leq C\left(\left[U_{0}, U_{0}, U_{0}\right]\right)$, which is false. Thus $(i, j) \neq(1,5)$ and hence $i \neq 1$. By symmetry $i \neq 6$.

Now assume $i=3$. Then $j \neq 1,4,6$ by the above. Since $X / O_{2}(X)$ $\cong S L(2, q) \times S L(3, q)$ we argue as in the above paragraph to show that $(i, j) \neq(3,2)$. Thus $j=5$. Then $U_{0} \leq O_{2}\left(P_{3}^{g} \cap P_{5}^{g p}\right) \cong Q_{3} Q_{5}$. From Table 
2 and (3.1) $Q_{3} Q_{5}$ has class $4, Z\left(Q_{3} Q_{5}\right)=U_{r_{14}} U_{r_{15}} U_{r_{16}}$ and $\left\langle U_{ \pm \alpha_{2}}, U_{ \pm \alpha_{4}}\right\rangle \cong$ $S L(3, q)$ acts irreducibly on $Z\left(Q_{3} Q_{5}\right)$. Since $O^{2^{\prime}}\left(P_{3,5} / Q_{3} Q_{5}\right) \cong\left\langle U_{ \pm \alpha_{1}}\right\rangle \times$ $\left\langle U_{ \pm \alpha_{2}}, U_{ \pm \alpha_{4}}\right\rangle \times\left\langle U_{ \pm \alpha_{6}}\right\rangle$, the preimage of the $S L(3, q)$ in $X$ covers the factor of $S L(3, q)$ in $P_{3}^{g} \cap P_{5}^{g p}$. However the preimage centralizes $\left[U_{0}, U_{0}, U_{0}\right]$. Consequently $\left[U_{0}, U_{0}, U_{0}\right] \cap Z\left(O_{2}\left(P_{3}^{g} \cap P_{5}^{g p}\right)\right)=1$. As $Q=O_{2}\left(P_{3}^{g} \cap P_{5}^{g p}\right) \sim$ $Q_{3} Q_{5},|Q|=\left|Q_{3} Q_{5}\right|=q^{31}$. Since $U_{0} \cap[Q, Q, Q, Q]=1, \mid U_{0} \Phi(Q) / \Phi(Q \mid \leq q$. Also $U_{0} \Phi(Q)<Q$, as $U_{0}<Q$. But then we must have $[X, Q / \Phi(Q)]=$ $U_{0} \Phi(Q) / \Phi(Q)<Q / \Phi(Q)$. However the preimage of the $S L(3, q)$ in $X / U_{0}$ covers the $S L(3, q)$ in $P_{3}^{g} \cap P_{5}^{g p} / Q$ and checking the action of $\left\langle U_{ \pm \alpha_{2}}, U_{ \pm \alpha_{4}}\right\rangle$ on $Q_{3} Q_{5} /\left(Q_{3} Q_{5}\right)^{\prime}$ we see that $\left[\left\langle U_{ \pm \alpha_{2}}, U_{ \pm \alpha_{4}}\right\rangle, Q_{3} Q_{5}\right]=Q_{3} Q_{5}$, which is a contradiction. Therefore $i \neq 3$ and by symmetry $i \neq 5$. We have proved that the only parabolic subgroup of $G$ containing $X$ is $P_{4}$.

Now $C_{G}(z)$ is contained in a parabolic subgroup by (2.3), so we have $C_{G}(z) \leq P_{4}$. Considering $C_{G}(z)$ acting on $\left[Q_{4}, Q_{4}\right] /\left[Q_{4}, Q_{4}, Q_{4}\right]$ we have $C_{G}(z) Q_{4} / Q_{4}<O^{2^{\prime}}\left(P_{4} / Q_{4}\right)$. However $X Q_{4} / Q_{4}$ is maximal in $O^{2^{\prime}}\left(P_{4} / Q_{4}\right)$, and so $X Q_{4}=C_{G}(z) Q_{4}$. Since $X \geq C_{U}(z)$ we have $X=C_{G}(z)$.

We now summarize our results.

(15.4) The maximal parabolic subgroups of $G$ containing the centralizers of the involutions in (15.1) are as follows:
i) $C_{G}(x) \leq P_{2}$
ii) $C_{G}(y) \leq P_{1}, P_{6}$
iii) $C_{G}(z) \leq P_{4}$.

In particular $G$ has precisely three classes of involutions.

(15.5) i) $C_{G}(x)=O^{2^{\prime}}\left(P_{2}\right)=Q_{2} L_{2}$, and $L_{2} \cong S L(6, q)$ acts irreducibly on $Q_{2} / Z\left(Q_{2}\right)$. The group $Q_{2}$ is special with center $U_{r}$. There is a cyclic group $W_{0} \leq H$ of order $q-1$ such that $\left[L_{2}, W_{0}\right]=1, L_{2} \cap W_{0}=Z\left(L_{2}\right)$, $P_{2}=C_{G}(x) W_{0}$, and $C_{G}\left(W_{0}\right)=W_{0} L_{2}$.

ii) $C_{G}(y)=U_{0}\left(L_{0} \times H_{0}\right)$, where $U_{0}=Q_{1} Q_{6}, L_{0}=\left\langle U_{\alpha_{3}}(c) U_{\alpha_{5}}(c), U_{\alpha_{4}}, U_{\alpha_{2}}\right.$, $\left.s_{3} s_{5}, s_{4}, s_{2}: c \in F_{q}\right\rangle \cong S p(6, q) \cong S O(7, q)$, and $H_{0}$ is cyclic of order $(q-1) /$ $(3, q-1)$. We have $\left[L_{0}, U_{0}\right]=U_{0}$. Also $U_{0}^{\prime}=Z\left(U_{0}\right)=Q_{1} \cap Q_{6}$ has order $q^{8}$ and $L_{1,6}$ acts on $Z\left(U_{0}\right)$ preserving a non-degenerate quadratic form in which $L_{0}$ is the centralizer of an anistropic 1-space containing $y$. $Z\left(C_{G}(y)\right)=P=\left\langle U_{\alpha}(c) U_{\beta}(c): c \in F_{q}\right\rangle$. There is an element $h \in H$ such that $U_{\alpha} U_{\beta}=P P^{n} . \quad$ Also $\left(U_{\alpha} U_{\beta}\right)^{\#}$ contains $2(q-1)$ conjugates of $x$ and $(q-1)^{2}$ conjugates of $y$.

iii) $C_{G}(z)=U_{0} L_{0}$ where $U_{0}, L_{0}$ are given in (15.2) and (15.3). The 
group $U_{0}$ has class 3 with $\left[U_{0}, U_{0}\right]=Q_{2}^{4} Q_{3}^{4}$ and $\left[U_{0}, U_{0}, U_{0}\right]=Q_{3}^{4} \leq Z\left(U_{0}\right)$. $L_{0} \cong S L(2, q) \times S L(3, q),\left[L_{0}, U_{0}\right]=U_{0}$ and $L_{0}$ acts irreducibly on $Z\left(U_{0}\right)$. The involution $z \in\left[U_{0}, U_{0}\right]-\left[U_{0}, U_{0}, U_{0}\right] . \quad Z\left(C_{G}(z)\right)=P=\left\langle U_{r}(c) U_{\delta}(c) U_{s}(c)\right.$ : $\left.c \in \boldsymbol{F}_{q}\right\rangle$. There is an element $h \in H$ such that $P P^{h}=U_{r} \times P$. For this $h$, $P P^{h}$ contains $q-1$ conjugates of $x, q-1$ conjugates of $y$, and $(q-1)^{2}$ conjugates of $z$.

Section 16. Centralizers of involutions in $E_{7}(q), q$ even.

Let $G=E_{7}(q)$ with $q=2^{a}$. Then $|H|=(q-1)^{7}, W=\left\langle s_{1}, \cdots, s_{7}\right\rangle$ and the action of $W$ on $\Delta$ in essentially as in $E_{6}(q)$ :

$$
\begin{aligned}
& \left(\alpha_{3}\right) s_{1}=\left(\alpha_{1}\right) s_{3}=\alpha_{1}+\alpha_{3} \\
& \left(\alpha_{4}\right) s_{2}=\left(\alpha_{2}\right) s_{4}=\alpha_{2}+\alpha_{4} \\
& \left(\alpha_{j}\right) s_{i}=\alpha_{i}+\alpha_{j} \text { if }|i-j|=1,\{i, j\} \neq\{1,2\},\{2,3\},
\end{aligned}
$$

with $\left(\alpha_{j}\right) s_{i}=\alpha_{j}$ for all other pairs $(i, j)$. As in $E_{6}(q)$ the only non-trivial commutator relation is in (3.1)(ii). Distinguish the $\operatorname{roots} r, \alpha, \beta, \gamma, \delta, \varepsilon, \varphi$, $\psi, \theta$ as in Table 3. From (12.9) we have

(16.1) Each involution in $E_{7}(q), q$ even, is conjugate to one of the following:
i) $x=U_{r}(1)$
ii) $y=U_{\alpha}(1) U_{\beta}(1)$
iii) $z=U_{r}(1) U_{\delta}(1) U_{\diamond}(1)$
iv) $u=U_{\varphi}(1) U_{\psi}(1) U_{\theta}(1)$
v) $v=U_{r}(1) U_{\theta}(1) U_{\psi}(1) U_{\varphi}(1)$.

We will determine the centralizers of $x, y, z, u$, and $v$. For $x=$ $U_{r}(1)$ we can use the results in Section 4 of [7] to check the following. $C_{G}(x)=Q_{1} L_{1}$ and $L_{1} \cong S O^{+}(12, q)$ acts irreducibly on $Q_{1} / Z\left(Q_{1}\right)=Q_{1} / U_{r}$. The parabolic subgroup $P_{1}=Q_{1}\left(L_{1} \times W_{0}\right)$ where $W_{0}$ is cyclic of order $q-1$ and is regular on $U_{r}^{*}$. Finally $C_{G}\left(W_{0}\right)=W_{0} \times L_{1}$.

Next consider $y=U_{\alpha}(1) U_{\beta}(1)$. The root groups $U_{s} \leq U$ with $s \in \Delta^{+}$ and such that $U_{s}$ does not commute with $U_{\alpha}(1)$ and $U_{\beta}(1)$ are as follows:

$U_{\alpha}(1) \quad\left\{s=\alpha_{2}, \alpha_{2}+\alpha_{4}, \alpha_{2}+\alpha_{3}+\alpha_{4}, \alpha_{1}+\alpha_{2}+\alpha_{3}+\alpha_{4}\right\} \quad\left\{\alpha+s=r_{24}, r_{25}, r_{26}, r_{27}\right\}$

$U_{\beta}(1)\left\{s=\alpha_{5}, \alpha_{4}+\alpha_{5}, \alpha_{3}+\alpha_{4}+\alpha_{5}, \alpha_{1}+\alpha_{3}+\alpha_{4}+\alpha_{5}\right\}\left\{\beta+s=r_{24}, r_{25}, r_{26}, r_{27}\right\}$.

It follows that 


$$
\begin{aligned}
C_{U}\left(U_{\alpha} U_{\beta}\right)=\left\langle U_{s}: s \neq \alpha_{2}, \alpha_{5}, r_{29}, r_{31}-\alpha_{2}, r_{30}, r_{33}-\alpha_{2}, r_{38}, r_{39}-\alpha_{2}\right\rangle \\
C_{U}(y)=\left\langle C_{U}\left(U_{\alpha} U_{\beta}\right), U_{\alpha_{2}}(c) U_{\alpha_{5}}(c), U_{r_{29}}(c) U_{r_{31-\alpha}}(c), U_{r_{30}}(c) U_{r_{33-\alpha_{2}}}(c),\right. \\
\left.\quad U_{r_{38}}(c) U_{r_{30}-\alpha_{2}}(c): c \in F_{q}\right\rangle .
\end{aligned}
$$

In particular $\left|U: C_{U}(y)\right|=q^{4}$ and $\left|C_{U}(y)\right|=q^{59}$.

Let $L \cong D_{5}(q) \times S L(2, q)$ be the Levi factor of $P_{6}$. Then let $L_{0}$ be the direct product of the $S L(2, q)$ factor with the fixed point group of the graph automorphism of the $D_{5}(q)$ factor. Then

$$
\begin{aligned}
L_{0} & =\left\langle U_{\alpha_{1}}, U_{\alpha_{3}}, U_{\alpha_{4}}, U_{\alpha_{2}}(c) U_{\alpha_{5}}(c), s_{1}, s_{3}, s_{4}, s_{2} s_{5}: c \in F_{q}\right\rangle \times\left\langle U_{ \pm \alpha_{7}}\right\rangle \\
& \cong S p(8, q) \times S L(2, q)
\end{aligned}
$$

and

$$
U_{0}=Q_{6} .
$$

Then one checks that $L_{0} \leq C(y), L \not C(y)$ and $X=U_{0} L_{0}=C_{P_{8}}(y)$. We first show that the only parabolic subgroup of $G$ containing $X$ is $P_{6}$. To see this first note that $\left|U_{0}\right|=\left|Q_{6}\right|=q^{42}$ so if $P=P_{i}^{q} \geq X$ and $\left|O_{2}(P)\right|$ $<q^{42}$, we must have $X$ contained in a parabolic subgroup of the form $P_{i}^{q} \cap P_{j}^{q p}$, for some $p \in P=P_{i}^{q}$. Since $X / O_{2}(X) \cong S p(8, q) \times S L(2, q)$ the only possible values for $i$ are $i=6,7$. If $i=7$, then $j$ must exist and hence $j=6$. But by orders $S p(8, q) \times S L(2, q) \nless P_{7}^{g} \cap P_{6}^{g p}$. So $i=6$ and $U_{0} \leq O_{2}(P)=Q_{6}^{g}$. But then $Q_{6}=U_{0}=Q_{6}^{g}$ and $g \in N_{G}\left(Q_{6}\right)=P_{6}$.

Consider the structure of $X \leq Q_{6} L_{6}$. We check using Table 2 and (3.1) that $U_{0}^{\prime}=Q_{6}^{\prime}=Q_{2}^{6}$. Construct a quadratic form on $U_{0}^{\prime}$ as follows. Elements in root groups are isotropic, $U_{0}^{\prime}=U_{r_{15}} U_{r_{22}} \times U_{r_{17}} U_{r_{28}} \times U_{r_{18}} U_{r_{25}}$ $\times U_{r_{20}} U_{r_{24}} \times U_{r_{21}} U_{r_{23}}$ is a decomposition into orthogonal hyperbolic planes and in each factor $g\left(U_{r_{i}}(s) U_{r_{j}}(t)\right)=s t$. Then it is easy to verify that $L_{8,7}=\left\langle U_{ \pm \alpha_{1}}, U_{ \pm \alpha_{2}}, U_{ \pm \alpha_{3}}, U_{ \pm \alpha_{4}}, U_{ \pm \alpha_{5}}\right\rangle \cong S O^{+}(10, q)$ preserves this form. Since $y$ isa nisotro pic $C_{L_{1,8}}(y) \cong S O(9, q) \cong S p(8, q)$. Consequently $C_{L_{8}}(y)=L_{0}$ and $X=C_{G}(y)$.

Using Table 3 and (3.1) we see that $\left[L_{0}, U_{0} / U_{0}^{1}\right]=U_{0} / U_{0}^{1}$, so $\left[L_{0}, U_{0}\right]$ $U_{0}$. If $q>2$, then $C(y)=C(y)^{\prime}$, while for $q=2, C(y)^{\prime}$ has index 2 in $C(y)\left(\operatorname{as}\left\langle U_{ \pm \alpha_{7}}\right\rangle \cong S_{3}\right)$. There is an element $w \in W$ such that $(\alpha, \beta) w=\left(\alpha_{1}, \alpha_{6}\right)$, so $\left(U_{\alpha} U_{\beta}\right)^{w} \leq\left\langle U_{ \pm \alpha_{1}}\right\rangle \times\left\langle U_{ \pm \alpha_{\alpha}}\right\rangle$ and $H$ is transitive $U_{\alpha}^{\sharp} U_{\beta}^{\sharp}$. So setting $P=$ $\left\langle U_{\alpha}(c) U_{\beta}(c): c \in F_{q}\right\rangle$, we have an element $h \in H$ such that $U_{\alpha} U_{\beta}=P P^{h}$. Then $P P^{h}$ contains $2(q-1)$ conjugates of $x$ and $(q-1)^{2}$ conjugates of y. Also $P=Z\left(C_{G} H\right)$. 
We next find $C_{G}(z), z=U_{\gamma}(1) U_{\delta}(1) U_{s}(1)$. As usual we begin with $C_{U}(z)$. The roots $s \in \Delta^{+}$such that $U_{s}$ does not centralize $U_{r}, U_{\tilde{o}}$, and $U_{\varepsilon}$ are as follows

$$
\begin{array}{lll}
U_{\gamma} & \left\{s=r_{1}, r_{2}, r_{3}, r_{4}, r_{5}, r_{6}\right\} \quad\left\{\gamma+s=r_{22}, r_{23}, r_{24}, r_{25}, r_{26}, r_{27}\right\} \\
U_{\delta} & \left\{s=\alpha_{4}, \alpha_{4}+\alpha_{5}, r_{29}, r_{31}, r_{35}, r_{41}\right\} & \left\{\delta+s=r_{20}, r_{21}, r_{23}, r_{24}, r_{26}, r_{27}\right\} \\
U_{\varepsilon} & \left\{s=\alpha_{6}, \alpha_{5}+\alpha_{6}, r_{28}, r_{32}, r_{34}, r_{40}\right\} & \left\{\varepsilon+s=r_{20}, r_{21}, r_{22}, r_{25}, r_{26}, r_{27}\right\}
\end{array}
$$

consequently $C_{U}\left(U_{\gamma} U_{\delta} U_{\varepsilon}\right)=\left\langle U_{s}: s \in \Delta^{+}\right.$not a root in 16.4 $\rangle$. In addition for $c \in \boldsymbol{F}_{q} C_{U}(z)$ contains the following elements of $U$.

$$
\begin{aligned}
& U_{\alpha_{7}}(c) U_{\alpha_{2}}(c), U_{r_{2}}(c) U_{r_{29}}(c), U_{r_{3}}(c) U_{r_{31}}(c), U_{r_{4}}(c) U_{r_{32}}(c), U_{r_{5}}(c) U_{r_{35}}(c), \\
& U_{r_{35}}(c) U_{r_{34}}(c), U_{r_{6}}(c) U_{r_{41}}(c), U_{r_{41}}(c) U_{r_{40}}(c), U_{\alpha_{4}}(c) U_{\alpha_{6}}(c), \\
& U_{\alpha_{4}+\alpha_{5}}(c) U_{\alpha_{5}+\alpha_{6}}(c) .
\end{aligned}
$$

We then obtain

$$
\begin{gathered}
C_{U}(z)=C_{Q_{3}}(z) C_{U_{\cap} L_{3}}(z), \quad \text { where } \\
C_{Q_{3}}(z)=\left\langle C_{U}\left(U_{\gamma} U_{\delta} U_{s}\right), U_{r_{5}}(c) U_{r_{35}}(c), U_{r_{34}}(c) U_{r_{35}}(c), U_{r_{6}}(c) U_{r_{40}}(c),\right. \\
\left.U_{r_{8}}(c) U_{r_{41}}(c): c \in F_{q}\right\rangle \\
C_{U_{\cap} L_{3}}(z)=\left\langle U_{\alpha_{1}}\right\rangle \times\left\langle U_{\alpha_{5}}, U_{\alpha_{4}}(c) U_{\alpha_{6}}(c), U_{\alpha_{2}}(c) U_{\alpha_{7}}(c), U_{\alpha_{4}+\alpha_{5}+\alpha_{6}},\right. \\
U_{\alpha_{2}+\alpha_{4}+\alpha_{5}+\alpha_{6}+\alpha_{7}}, U_{\alpha_{4}+\alpha_{5}}(c) U_{\alpha_{5}+\alpha_{6}}(c), U_{r_{2}}(c) U_{r_{29}}(c), \\
\left.U_{r_{3}}(c) U_{r_{31}}(c), U_{r_{4}}(c) U_{r_{32}}(c)\right\rangle .
\end{gathered}
$$

It is easy to check from (3.1) that $C_{U \cap L_{3}}(z)$ is isomorphic in a natural way to a Sylow 2-subgroup of $S L(2, q) \times S p(6, q)$. We set

$$
\begin{aligned}
U_{0} & =C_{Q_{3}}(z) \\
L_{0} & =\left\langle C_{U \cap L_{3}}(z), s_{1}, s_{5}, s_{4} s_{6}, s_{2} s_{7}\right\rangle \\
& =\left\langle U_{ \pm \alpha_{1}}\right\rangle \times\left\langle U_{\alpha_{5}}, U_{\alpha_{4}}(c) U_{\alpha_{6}}(c), U_{\alpha_{2}}(c) U_{\alpha_{7}}(c), s_{5}, s_{4} s_{6}, s_{2} s_{7}: c \in F_{q}\right\rangle .
\end{aligned}
$$

Then $U_{0} L_{0} \leq C_{P_{3}}(z)$ and $L_{0}=\left\langle U_{ \pm \alpha_{1}}\right\rangle \times\left\langle L_{00}\right\rangle$, where $L_{00}$ is the fixed point group under the graph automorphism of the Levi factor of $P_{1,3}$. It follows that

$$
L_{0} \cong S L(2, q) \times S p(6, q) .
$$

We use Table 3 and (3.1) to check that $\left|U_{0}\right|=q^{45}, U_{0}^{\prime}=Q_{2}^{3} Q_{3}^{3}=Q_{3}^{\prime}$, and $\left[U_{0}, U_{0}, U_{0}\right]=Q_{3}^{3}=\left[Q_{3}, Q_{3}, Q_{3}\right]$. Also we check that $\left[L_{0}, U_{0} / U_{0}^{\prime}\right]=U_{0} / U_{0}^{\prime}$, so that $\left[L_{0}, U_{0}\right]=U_{0}$. We have $\left\langle U_{ \pm \alpha_{1}}\right\rangle \leq L_{0}$ acting irreducibly on 
$\left[U_{0}, U_{0}, U_{0}\right]$. To show that $C_{P_{3}}(z)=U_{0} L_{0}$ we will use the following lemma.

(16.8). Let $M=\left\langle U_{ \pm \alpha_{1}}, \cdots, U_{ \pm 2 n-1}\right\rangle \cong P S L(2 n, q)$ and $M_{0}=\left\langle U_{\alpha_{1}}(c) U_{\alpha_{2 n-1}}(c)\right.$, $\left.\cdots U_{\alpha_{n-1}}(c) U_{\alpha_{n+1}}(c), U_{\alpha_{n}}, s_{1} s_{2 n-1}, \cdots s_{n-1} s_{n+1}, s_{n}: c \in \boldsymbol{F}_{q}\right\rangle \cong S p(2 n, q)$ be the natural embedding of $S p(2 n, q)$ in $S L(2 n, q)$. Then $M_{0}$ is maximal in $M$.

Proof. We sketch a proof by induction. Let $V$ be the natural module for $S L(2 n, q)$. It is easy to produce a non-degenerate alternating form on $V$ left invariant by $M_{0}$. It follows that $M_{0}$ is transitive on $V^{\sharp}$. Suppose that $M_{0}<T<M$ and let $P_{1}$ be the stabilizer in $M$ of a 1-space in $V$. Then $M=M_{0} P_{1}$ and $T=M_{0}\left(P_{1} \cap T\right)$. The structure of $P_{1}$ is well-known: $P_{1}=Q L H_{0}$, where $Q$ is elementary of order $q^{2 n-1}, L \cong$ $S L(2 n-1, q)$ acts in the natural way on $Q$, and $H_{0}$ is cyclic of order $q-1$ normalizing $L$ and $Q$. Also $P_{1} \cap M_{0}=Q_{0}\left(L_{0} \times H_{1}\right)$ where $Q_{0}$ is elementary of order $q^{2 n-1}$ and $L_{0} \cong S p(2 n-2, q)$ acts on $Q_{0} / Q_{0} \cap Q$ in the natural way. Here $Q_{0} \cap Q$ is a 1-space in $Q$. If $T \cap Q>M_{0} \cap Q=$ $Q_{0} \cap Q$, then since $L_{0}$ is irreducible on $Q / Q_{0} \cap Q$ we have $Q \leq T$. But then $P_{1}=N_{M}(Q)$ and $M=M_{0} P_{1}=T P_{1}$ implies that $M=Q^{M}=Q^{T} \leq T$, a contradiction. So $T \cap Q=Q_{0} \cap Q$ and $T \cap P_{1}$ stabilizes a 1-space of $Q$. Consequently $T \cap P_{1} \leq Q L_{1} H$ where $L_{1}$ is a maximal parabolic subgroup of $L$. But $Q L_{1} H=P_{1,2}$ is a parabolic subgroup of $M$ corresponding to the stabilizer of a 1-space of $V$ and a hyperplane containing it. Now $P_{1,2}=Q Q_{0}\left(S L(2 n-2, q) H_{00}\right)$ where $H_{00}$ is abelian. If $T \cap P_{1}$ covers the $S L(2 n-2, q)$ then we have $T B=M$ where $B \geq H_{00} Q_{0} Q$ is a Borel group of $M$. This contradicts the main theorem of [19]. Otherwise we have, by induction (or without any argument if $n=2), T \cap P_{1} \leq\left(M_{0} \cap P_{1}\right) H_{00}$ and hence $T=M_{0} K$ where $K$ can be chosen as a subgroup of $H$. From here it is easy to see that $K \leq M_{0}$ and $T=M_{0}$, a contradiction.

Since $O^{2^{\prime}}\left(P_{3} / Q_{3}\right) \cong S L(2, q) \times S L(6, q)$ we can use (16.8) to verify that $U_{0} L_{0}=C(z) \cap O^{2^{\prime}}\left(P_{3}\right)$ and from here we obtain $U_{0} L_{0}=C_{P_{3}}(z)$.

We claim that $P_{3}$ is the only parabolic subgroup of $G$ that contains $U_{0} L_{0}$ and that $U_{0} L_{0}=C_{G}(z)$. The second statement follows from the first since we know, from (2.3), that $C_{G}(z)$ is contained in a parabolic subgroup of $G$. So suppose that $X=U_{0} L_{0} \leq P_{i}^{q}$. By order considerations for $X / U_{0} \cong S L(2, q) \times S p(6, q)$, we have $i \neq 2,4,5$. Also we have $\left|U_{0}\right|=q^{45}$ so $U_{0} \not Q_{i}^{g}$ unless $i=3$. If $i=3$ then $U_{0} \leq Q_{3}^{g}$ and $P_{3}=N_{G}\left(\left[Q_{3}, Q_{3}, Q_{3}\right]\right)$ $=N_{G}\left(\left[U_{0}, U_{0}, U_{0}\right]\right)=N_{G}\left(\left[Q^{g}, Q^{g}, Q^{g}\right]\right)=P_{3}^{g}$, so the claim holds. Suppose 
then that $i \neq 3$ as well. If $i=7$, then $U_{0} \nless Q_{i}^{g}$ and we have $U_{0} \leq P_{i}^{g} \cap P_{j}^{g p}$ where $j=1$ or 6 and $p \in P_{i}^{q}$. In either case we still cannot have $U_{0} \leq$ $\left(P_{i}^{g} \cap P_{j}^{g p}\right)$. Consequently we must have $U_{0} L_{0} \leq P_{7}^{g} \cap P_{6}^{g p} \cap P_{1}^{g p_{1}}$ for some $p_{1} \in P_{7}^{g}$. Again order considerations rule this out. So $i \neq 7$. Similarly $i \neq 1,6$. We now have the claim, so $C_{G}(z)=U_{0} L_{0}$.

We set $P=\left\langle U_{r}(c) U_{\delta}(c) U_{s}(c): c \in F_{q}\right\rangle$. Then $P=Z\left(C_{G}(z)\right)$. As in other cases we have $H$ transitive on $U_{\gamma}^{\sharp} U_{\delta}^{\sharp} U_{s}^{\#}$. Consequently there is an element $h \in H$ such that $h$ centralizes $U_{\delta} U_{s}$ but not $U_{r}$. Then $P P^{h}=P \times U_{r}$ and $P P^{h}$ contains $q-1$ conjugates of $x, q-1$ conjugates of $y$, and $(q-1)^{2}$ conjugates of $z$. This completes our discussion of $C_{G}(z)$.

For $u=U_{\varphi}(1) U_{\psi}(1) U_{\theta}(1)$ we first list the roots $s \in \Delta^{+}$such that $U_{s}$ does not commute with $U_{\varphi}, U_{\psi}$, or $U_{\theta}$.

$$
\begin{array}{ll}
U_{\varphi} \quad\left\{s=\alpha_{6}, \alpha_{3}, \alpha_{3}+\alpha_{4}, \alpha_{3}+\alpha_{4}+\alpha_{5}+\alpha_{6}, r_{30}, r_{34}, r_{36}, r_{45}\right\} \\
& \left\{\varphi+s=r_{17}, r_{16}, r_{19}, r_{21}, r_{22}, r_{24}, r_{25}, r_{27}\right\} \\
U_{\psi} \quad\left\{s=\alpha_{5}, \alpha_{5}+\alpha_{6}, \alpha_{4}+\alpha_{5}, \alpha_{4}+\alpha_{5}+\alpha_{6}, r_{31}, r_{32}, r_{37}, r_{44}\right\} & \\
& \left\{\psi+s=r_{16}, r_{18}, r_{19}, r_{20}, r_{22}, r_{23}, r_{26}, r_{27}\right\} \\
U_{\theta} \quad\left\{s=\alpha_{1}, \alpha_{1}+\alpha_{3}, \alpha_{1}+\alpha_{3}+\alpha_{4}, \alpha_{1}+\alpha_{3}+\alpha_{4}+\alpha_{5}, r_{38}, r_{39}, r_{41}, r_{43}\right\} & \\
& \left\{\varepsilon+s=r_{17}, r_{18}, r_{20}, r_{21}, r_{23}, r_{24}, r_{25}, r_{26}\right\} .
\end{array}
$$

So $C_{U}\left(U_{\varphi} U_{\psi} U_{\theta}\right)=\left\langle U_{s}: s\right.$ not as in 16.9) $\rangle$. In addition, for each $c \in \boldsymbol{F}_{q}$, $C_{U}(u)$ contains the elements

$$
\begin{aligned}
& U_{\alpha_{6}}(c) U_{\alpha_{1}}(c), U_{\alpha_{3}}(c) U_{\alpha_{5}}(c), U_{\alpha_{3}+\alpha_{4}}(c) U_{\alpha_{4}+\alpha_{5}}(c), U_{\alpha_{1}+\alpha_{3}}(c) U_{\alpha_{5}+\alpha_{8}}(c) \\
& U_{\alpha_{4}+\alpha_{5}+\alpha_{8}}(c) U_{\alpha_{1}+\alpha_{3}+\alpha_{4}}(c), U_{\alpha_{3}+\alpha_{4}+\alpha_{5}+\alpha_{6}}(c) U_{\alpha_{1}+\alpha_{3}+\alpha_{4}+\alpha_{5}}(c), U_{r_{30}}(c) U_{r_{31}}(c), \\
& U_{32}(c) U_{r_{38}}(c), U_{r_{34}}(c) U_{r_{39}}(c), U_{r_{38}}(c) U_{r_{41}}(c), U_{r_{37}}(c) U_{r_{43}}(c), U_{r_{45}}(c) U_{r_{44}}(c)
\end{aligned}
$$

We note that $U_{0}=Q_{7} \leq C_{U}\left(U_{\varphi} U_{\psi} U_{\varepsilon}\right) \leq C_{U}(u)$ and that $C_{U}(u)=\left\langle C_{U}\left(U_{\varphi} U_{\psi} U_{\theta}\right)\right.$, $U_{r_{i}}(c) U_{r_{j}}(c): c \in \boldsymbol{F}_{q},(i, j)$ as in (16.10) $\rangle$. In particular $\left|U_{0}\right|=q^{27}$ and $\left|C_{U}(u)\right|=q^{51}$.

The Levi factor $L$ of $P_{7}$ is isomorphic to $E_{6}(q)$. Let $L_{0}$ be the group of fixed points under the graph automorphism of $L$. Then

$$
L_{0}=\left\langle U_{\alpha_{2}}, U_{\alpha_{4}}, U_{\alpha_{3}}(c) U_{\alpha_{5}}(c), U_{\alpha_{1}}(c) U_{\alpha_{6}}(c), s_{2}, s_{4}, s_{3} s_{5}, s_{1} s_{6}: c \in F_{q}\right\rangle .
$$

Also we see that

$$
\begin{gathered}
L_{0} \cong F_{4}(q) \\
X=U_{0} L_{0} \leq C_{P_{7}}(u) .
\end{gathered}
$$


We first claim that $P_{7}$ is the only parabolic subgroup of $G$ containing $X$. Since $X / O_{2}(X) \cong L_{0} \cong F_{4}(q)$ we see by order considerations that if $X \leq P_{i}^{g}$, then $i=1$ or 7 . Also we must have $U_{0} \leq O_{2}\left(P_{i}^{g}\right)$, for otherwise $X \leq P_{i}^{q} \cap P_{j}^{g p}$ for some $i \neq j, p \in P_{i}^{g}$. If $i=1$, then $O_{2}\left(P_{i}^{g}\right) \cong Q_{1}$ which is special of order $q^{33}$ with center $U_{r}$. If $M$ has index 2 in $U_{r}$, then $Q_{1} / M$ is extra-special of order $2 q^{32}$ and from here it is clear that $O_{2}\left(P_{\imath}^{q}\right)$ cannot contain an abelian group of order $q^{27}=\left|Q_{7}\right|$. Consequently $i=7, U_{0} \leq$ $O_{2}\left(P_{7}^{g}\right)$ and by order $Q_{7}=U_{0}=O_{2}\left(P_{7}^{g}\right)=Q_{7}^{g}$. Then $g \in N\left(Q_{7}\right)=P_{7}$ and the claim holds.

Since $C_{G}(u)$ is contained in a parabolic subgroup of $G$ we must have $C_{G}(u) \leq P_{7}$. The following lemma forces $C_{G}(u)=X$.

(16.13) Let $L_{0} \cong F_{4}(q)$ be embedded in $M=L_{7} \cong E_{6}(q)$ as in (16.12). Then $L_{0}$ is maximal in $M$.

Proof. We outline a proof in the following way. First check that if $L_{0}<T<M$ and if $H \cap T>H \cap L_{0}$ then we have the contradiction that $T=M$. We will use Guterman [14] to obtain a contradiction. Let $t_{1}, t_{2}, t_{1} t_{2}$ be the central involutions given in (13.1) i), ii), iii) respectively. Then check that in $E_{6}(q), t_{1} \sim x, t_{2} \sim y, t_{1} t_{2} \sim y$, where $x, y$ are as in (15.1).

The idea is to show that $C_{T}\left(t_{1}\right)=C_{L_{0}}\left(t_{1}\right), C_{T}\left(t_{2}\right)=C_{L_{0}}\left(t_{1}\right)$, and $C_{T}\left(t_{1} t_{2}\right)$ $=C_{L_{0}}\left(t_{1} t_{2}\right)$. For then [14] shows that $T \cong F_{4}(q)$ and consequently $L_{0}=T$, a contradiction. Using the result in $\S 13$ and $\S 15$ we see that in each case either the centralizers behave as desired or $T \geq U_{\alpha_{i}}$ for $i=1,3,5$, or 6. But this implies that $T=M$. For example if $U_{\alpha_{1}} \leq T$, then $T \geq$ $\left\langle U_{\alpha_{1}}, U_{\alpha_{1}}^{s_{1} s_{6}}\right\rangle=\left\langle U_{ \pm \alpha_{1}}\right\rangle$ and $s_{1} \in T$. Also $T \geq\left\langle U_{\alpha_{1}} U_{-\alpha_{1}}\right\rangle^{s_{3} s_{5} s_{1} s_{6}}=\left\langle U_{ \pm \alpha_{3}}\right\rangle$ and $s_{3} \in T$. Then $s_{1}, \cdots, s_{6} \in T$ and clearly this forces $T=M$.

We have now found $C_{G}(u)=U_{0} L_{0}$ and we need the action of $L_{0}$ on $U_{0}=Q_{7}$. Set $P=\left\langle U_{\varphi}(c) U_{\psi}(c) U_{\theta}(c): c \in F_{q}\right\rangle$. Then $L_{0}$ centralizes $P \leq Q_{7}$ and $P=Z\left(C_{G}(u)\right)$. Also we argue as in previous situations to find $h \in H$ such that $P P^{h}=U_{\varphi} \times P$. Then $P P^{h}$ contains $q-1$ conjugates of $x$, $q-1$ conjugates of $y$, and $(q-1)^{2}$ conjugates of $u$.

Let $S=\left\langle U_{\varphi}(c) U_{\psi}(c), U_{\varphi}(c) U_{\theta}(c): c \in \boldsymbol{F}_{q}\right\rangle$ and set

$$
U_{1}=\left\langle S, U_{s}: s \in \mathscr{L}_{7}, s \neq \varphi, \psi, \theta\right\rangle .
$$

Then checking the action of $L_{0}$ on $U_{0}=Q_{7}$ we see that

$$
U_{0} L_{0}=U_{1} L_{1} \times P
$$


and

$$
\left(U_{0} L_{0}\right)^{\prime}=U_{1} L_{1}
$$

The final centralizer to consider in this section is $C_{G}(v)$, where $v=$ $U_{r}(1) U_{\varphi}(1) U_{\psi}(1) U_{\theta}(1)=U_{r}(1) u$. The roots $s \in \Delta^{+}$with $U_{s} \not \subset C\left(U_{\varphi} U_{\psi} U_{\theta}\right)$ are listed in (16.9) and for $U_{r}$ in (16.4). Each root $s$ in (16.9) satisfies $U_{s} \leq C\left(U_{\gamma}\right)$, so the elements in (16.10) centralize $v$. In addition for each $c \in F_{q}$ the following are in $C(v)$

$$
\begin{aligned}
& U_{r_{1}}(c) U_{r_{30}}(c), U_{r_{2}}(c) U_{r_{32}}(c), U_{r_{3}}(c) U_{r_{34}}(c) \\
& U_{r_{4}}(c) U_{r_{36}}(c), U_{r_{5}}(c) U_{r_{37}}(c), U_{r_{6}}(c) U_{r_{45}}(c) .
\end{aligned}
$$

It follows that $\left|C_{U}\left(U_{r} U_{\varphi} U_{\psi} U_{\theta}\right)\right|=q^{63-24-6}=q^{33}$ and $\left|C_{U}(v)\right|=q^{33+18}=q^{51}$. Notice that $C_{U}(v) \geq C_{U}\left(U_{\gamma} U_{\varphi} U_{\psi} U_{\theta}\right) \geq Q_{2} \cap Q_{7}, C_{Q_{7}}(v)=Q_{2} \cap Q_{7}$, and $C_{Q_{2}}\left(U_{\gamma} U_{\varphi} U_{\psi} U_{\theta}\right)=\left(Q_{2} \cap Q_{7}\right)\left\langle U_{s}: s=r_{28}, r_{29}, r_{33}, r_{35}, r_{40}, r_{42}, r_{44}, r_{47}, r_{48}\right\rangle$. Setting $U_{0}=C_{Q_{2} Q_{7}}(v)$ w.e have

$$
\begin{gathered}
U_{0}=C_{Q_{2}}\left(U_{r} U_{\varphi} U_{\psi} U_{\theta}\right)\left\langle U_{r_{i}}(c) U_{r_{j}}(c): c \in F_{q},(i, j) \text { as in }(16.16)\right. \text { or } \\
(i, j)=(30,31),(32,38),(34,39),(36,41),(37,43),(45,44)\rangle .
\end{gathered}
$$

Setting

$$
L_{0}=\left\langle U_{\alpha_{4}}, U_{\alpha_{3}}(c) U_{\alpha_{5}}(c), U_{\alpha_{1}}(c) U_{\alpha_{8}}(c), s_{4}, s_{3} s_{5}, s_{1} s_{6}=c \in \boldsymbol{F}_{q}\right\rangle,
$$

we have from the work on $C_{G}(u)$, that $L_{0} \cong B_{3}(q) \cong S p(6, q)$ as it occurs naturally in a parabolic subgroup of $\left\langle U_{ \pm \alpha_{2}}, L_{0}\right\rangle$, which was proved to be naturally isomorphic to $F_{4}(q)$. Now set $X=U_{0} L_{0}$. Then $X=C(v) \cap P_{2,7}$.

We note that $L_{0}$ normalizes $U_{0}$ and we consider the structure of $X$. First use Table 3 and (3.1) to show that $\left[U_{0}, U_{0}\right]=\left\langle U_{s}, U_{\varphi}(c) U_{\psi}(c)\right.$, $\left.U_{\varphi}(c) U_{\theta}(c): c \in F_{q}, s \in\left(\mathscr{L}_{7} \cap \mathscr{L}_{2}\right) \cup \mathscr{L}_{2}^{2}, s \neq r_{13}, r_{14}, r_{15}\right\rangle$ and also that $\left[U_{0}, U_{0}, U_{0}\right]$ $=Q_{2}^{2} \cap Q_{7}=\left[Q_{2} Q_{7}, Q_{2} Q_{7}, Q_{2} Q_{7}\right]$.

We note that $Q_{2}^{2}=U_{r} \times\left(Q_{2}^{2} \cap Q_{7}\right)$ and that $L_{0}$ acts on $Q_{2}^{2} \cap Q_{7}$ and centralizes $U_{r}$. Let $P=\left\langle U_{r}(c) U_{\varphi}(c) U_{\psi}(c) U_{\theta}(c): c \in F_{q}\right\rangle$ and $P_{0}=$ $\left\langle U_{\varphi}(c) U_{\psi}(c) U_{\theta}(c): c \in F_{q}\right\rangle$. Then we check that $\left[L_{0}, U_{0} / U_{0}^{\prime}\right] \times P_{0} U_{0} / U_{0}^{\prime}=$ $U_{0} / U_{0}^{\prime}$, so that $U_{0}=\left[L_{0}, U_{0}\right] \times P_{0}$ and $X^{\prime}=\left[L_{0}, U_{0}\right] L_{0}=X^{\prime \prime}$.

As in other cases we obtain $H$ transitive on $U_{r}^{\#} U_{\varphi}^{\sharp} U_{\psi}^{\#} U_{\theta}^{\#}$, so there is an element $h \in H$ such that $h$ centralizes $U_{\varphi} U_{\psi} U_{\theta}$ but not $U_{r}$. Then $P P^{h}=U_{\gamma} \times P$ and $P P^{h}$ contains $q-1$ conjugates of $x, q-1$ conjugates of $u$, and $(q-1)^{2}$ conjugates of $v$. Moreover we can check that $P=Z(X)$. 
We claim that the only parabolic subgroups of $G$ that contain $X$ are $P_{2}$ and $P_{7}$. Say $X \leq P=P_{i}^{q}$. Since $X$ involves $S p(6, q), i \neq 4,5$. Say $i=1$. Since $\left|Q_{1}\right|=q^{33}$ and $\left|U_{0}\right|=q^{42}$, we have $U_{0} \not \leq O_{2}(P)$ and hence $X$ $\leq P_{i}^{g} \cap P_{j}^{g p}$ for some $j \neq i$ and $p \in P$. The only possible values for $j$ are $j=2,3,6$ or 7 . Say $j=2$. Then we must have $U_{0} \not \leq O_{2}\left(P_{i}^{g} \cap P_{j}^{q p}\right) \sim Q_{1} Q_{2}$. We observe that $\left|U_{0}^{\prime}\right|=q^{21}$ and that $U_{0}^{\prime}$ is abelian. Consequently, since $Q_{1}$ is special with center $U_{r}$ and $\left|Q_{1}\right|=q^{33}$ we cannot have $U_{0}^{\prime}$ contained in $Q_{1}$. However $Q_{2} Q_{1} / Q_{1}$ is abelian, so the case is out. The same argument works for $j=3,6,7$. Thus $i \neq 1$. If $i=3$, then since $L_{3} \cong$ $S L(2, q) \times S L(6, q)$ we must have $U_{1}=\left[U_{0}, L_{0}\right] \leq O_{2}\left(P_{3}^{g}\right) \cong Q_{3}$. But $\left|\left[Q_{3}, Q_{3}, Q_{3}\right]\right|=q^{2}$ while $\left|\left[U_{1}, U_{1}, U_{1}\right]\right|=q^{6}$. So $i \neq 3$. If $i=2$ then $X \not \leq$ $O_{2}(P)$ and $X \leq P_{2}^{g} \cap P_{j}^{g p}$ for some $j \neq 1,2,3,4,5$. As $X / U_{0} \cong S p(6, q)$ we must have $j=7$ and $U_{0} \leq O_{2}\left(P_{2}^{g} \cap P_{j}^{g p}\right)=Q$. Then order considerations show that $\left[U_{0}, U_{0}, U_{0}\right]=[Q, Q, Q]$ so that $g \in N\left(\left[Q_{2} Q_{7}, Q_{2} Q_{7}, Q_{2} Q_{7}\right]\right)=P_{2,7} \leq P_{2}$ and $P_{2}^{g}=P_{2}$. Next we assume that $i=6$. Then since $O_{2}(P) \sim Q_{6}$ has class 2 we must have $U_{0} \npreceq O_{2}(P)$ and so $X \leq P_{6}^{g} \cap P_{7}^{g p}$, some $p \in P$. Then $U_{0} \leq O_{2}\left(P_{6}^{g} \cap P_{7}^{g p}\right) \sim Q_{6} Q_{7}$. Since $\left|Q_{6} Q_{7} / Q_{6}\right|=q$ and $L_{6,7}$ is trivial on $Q_{6} Q_{7} / Q_{6}$, we must have $\left[U_{0}, L_{0}\right] \leq Q_{6}$. However $\left[U_{0}, L_{0}\right]$ has class 3 . This is impossible, showing that $i \neq 6$. Finally $i=7$ forces $X \leq P_{7}^{g} \cap P_{2}^{q p}$ and we proved earlier that this forces $g p \in P_{2} \cap P_{7}$ and as $p \in P_{7}^{g}$, we have $P=$ $P_{7}$. At this point the claim is proved.

Since $C_{G}(v) \leq P$ for some parabolic subgroup $P$ of $G, C_{G}(v) \leq P_{2}$ or $P_{7}$. We will show that $C_{G}(v)$ is contained in $P_{2} \cap P_{7}$. If $C_{G}(v) \leq P_{7}$ then since $v \notin Q_{7}$, we have $C_{G}(v) Q_{7} / Q_{7}$, centralizing an involution in $P_{7} / Q_{7}$ and this forces $C_{G}(v) \leq P_{2} \cap P_{7}$. Suppose that $C_{G}(v) \leq P_{2}$. It is easily verified from (3.1) and Table 3 that $L_{2}$ acts on $Q_{2}^{2}$ as on the natural module for $S L(7, q)$. Also $X Q_{2} / Q_{2}$, viewed as a subgroup of $L_{2}$, fixes the hyperplane $Q_{2}^{2} \cap Q_{7}$ of $Q_{2}^{2}$ and is transitive on the remaining hyperplanes. If $C_{G}(v)$ does not fix $Q_{2}^{2} \cap Q_{7}$, then it follows that $C_{G}(v)$ is 2-transitive on the set of hyperplanes in $Q_{2}^{2}$ and this forces $\left(Q_{7} Q_{2} / Q_{2}\right)^{C_{G}(v)}=\left(Q_{7} Q_{2} / Q_{2}\right)^{P_{2}}=L_{2} Q_{2} / Q_{2}$ to be in $C_{G}(v) Q_{2} / Q_{2}$. But this is impossible as $L_{2}$ does not fix $v Q_{2}^{\prime} / Q_{2}^{\prime}$. Thus $C_{G}(v)$ stabilizes $Q_{2}^{2} \cap Q_{7}$ and $C_{G}(v) \leq P_{2} \cap P_{7}$ as desired.

Since $C_{G}(v) \leq P_{2} \cap P_{7}$ and $L_{2,7} \cong S L(6, q)$ we apply (16.8) to see that $X=C_{G}(v)$. We have now completed our discussion of $E_{7}(q)$ and we list our results below.

(16.19) The maximal parabolic subgroups containing the centralizers of 
the involutions in (16.1) are as follows:
i) $C_{G}(x) \leq P_{1}$
ii) $C_{G}(y) \leq P_{6}$
iii) $C_{G}(z) \leq P_{3}$
iv) $C_{G}(u) \leq P_{7}$
v) $C_{G}(v) \leq P_{2}, P_{7}$.

In particular, no two of the involutions $x, y, z, u, v$ are conjugate.

(16.20) The structure of the centralizers of the involutions on (16.1) are as follows:

i) $C_{G}(x)=Q_{1} L_{1}$ and $L_{1} \cong S O^{+}(12, q)$ acts irreducibly on $Q_{1} / Z\left(Q_{1}\right)=$ $Q_{1} / U_{r}$. Also $P_{1}=Q_{1}\left(L_{1} \times W_{0}\right)$, where $W_{0} \leq H$ is cyclic of order $q-1$ and $C_{G}\left(W_{0}\right)=W_{0} \times L_{1}$.

ii) $C_{G}(y)=U_{0} L_{0}$, where $U_{0}=Q_{6}, L_{0} \cong S p(8, q) \times S L(2, q)$ and $L_{0}=$ $\left\langle U_{\alpha_{1}}, U_{\alpha_{3}}, U_{\alpha_{4}}, U_{\alpha_{2}}(c) U_{\alpha_{5}}(c), s_{1}, s_{3}, s_{4}, s_{2} s_{5}: c \in F_{q}\right\rangle \times\left\langle U_{ \pm \alpha_{7}}\right\rangle . \quad\left[L_{0}, U_{0}\right]=U_{0}, C_{G}(y)$ $=C_{G}(y)^{\prime}$ if $q>2$, and $C_{G}(y)^{\prime}$ has index 2 in $C_{G}(y)$ if $q=2$. The group $L_{6,7} \cong S O^{+}(10, q)$ acts on $\left[U_{0}, U_{0}\right]=Z\left(U_{0}\right)$ and preserves a non-degenerate quadratic form. In this action $L_{0} \cap L_{6,7}$ is the stabilizer of the anisotropic 1-space $P=\left\langle U_{\alpha}(c) U_{\beta}(c): c \in F_{q}\right\rangle=Z\left(C_{G}(y)\right)$. There is an element $h \in H$ such that $P P^{h}=U_{\alpha} U_{\beta}$ contains $2(q-1)$ conjugates of $x$ and $(q-1)^{2}$ conjugates of $y$.

iii) $C_{G}(z)=U_{0} L_{0}$, where $U_{0} \leq Q_{3}$ and $L_{0} \leq L_{3}$ are given in (16.7). We have $L_{0} \cong S L(2, q) \times S p(6, q),\left[L_{0}, U_{0}\right]=U_{0}$, and $U_{0}$ has class 3 with $\left[U_{0}, U_{0}, U_{0}\right]=Q_{3}^{3} . \quad C_{G}(z)^{\prime}=C_{G}(z)$ if $q>2$ and $C_{G}(z)^{\prime}$ has index 2 in $C_{G}(z)$ if $q=2 . \quad Z\left(C_{G}(z)\right)=P=\left\langle U_{r}(c) U_{\delta}(c) U_{s}(c): c \in F_{q}\right\rangle$ and there is an element $h \in H$ such that $P P^{h}=P \times U_{r}$ contains $q-1$ conjugates of $x, q-1$ conjugates of $y$, and $(q-1)^{2}$ conjugates of $z$.

iv) $C_{G}(u)=U_{0} L_{0}$, where $U_{0}=Q_{7}, F_{4}(q) \cong L_{0} \leq L_{7}$, and $L_{0}=\left\langle U_{\alpha_{2}}, U_{\alpha_{4}}\right.$, $\left.U_{\alpha_{3}}(c) U_{\alpha_{5}}(c), U_{\alpha_{1}}(c) U_{\alpha_{6}}(c), s_{2}, s_{4}, s_{3} s_{5}, s_{1} s_{6}: c \in F_{q}\right\rangle$. Let $P=\left\langle U_{\varphi}(c) U_{\psi}(c) U_{\theta}(c)\right.$ : $\left.c \in F_{q}\right\rangle$. Then $P=Z\left(C_{G}(u)\right), U_{0}=\left[L_{0}, U_{0}\right] \times P$, and $C_{G}(v)=\left[L_{0}, U_{0}\right] L_{0} \times P$ has derived group $\left[L_{0}, U_{0}\right] L_{0}$. There is an element $h \in H$ such that $P P^{h}$ $=U_{\varphi} \times P$ contains $q-1$ conjugates of $x, q-1$ conjugates of $y$, and $(q-1)^{2}$ conjugates of $u$.

v) $C_{G}(v)=U_{0} L_{0}$, where $U_{0} \leq Q_{2} Q_{7}$ is given in (16.17) and $S p(6, q)$ $\cong L_{0} \leq L_{2,7}$ is given in (16.18). $U_{0}$ has class 3 with $\left[U_{0}, U_{0}, U_{0}\right]=Q_{7} \cap Q_{2}^{2}$ $=\left[Q_{2} Q_{7}, Q_{2} Q_{7}, Q_{2} Q_{7}\right]$. Set $P=\left\langle U_{r}(c) U_{\varphi}(c) U_{\psi}(c) U_{\theta}(c): c \in \boldsymbol{F}_{q}\right\rangle$ and $P_{0}=$ $\left\langle U_{\varphi}(c) U_{\psi}(c) U_{\theta}(c): c \in F_{q}\right\rangle$. Then $U_{0}=\left[L_{0}, U_{0}\right] \times P_{0}$ and $C_{G}(v)^{\prime}=\left[L_{0}, U_{0}\right] L_{0}$. 
There is an element $h \in H$ such that $P P^{h}=U_{r} \times P$ contains $q-1$ conjugates of $x, q-1$ conjugates of $u$, and $(q-1)^{2}$ conjugates of $v$. Also $P=Z\left(C_{G}(v)\right)$.

Section 17. Centralizers of involutions in $E_{8}(q), q$ even.

Let $G=E_{8}(q), q=2^{a}$. Then $|H|=(q-1)^{8}, W=\left\langle s_{1}, \cdots, s_{8}\right\rangle$ and the action of $W$ on $\Delta$ is determined by

$$
\begin{aligned}
& \left(\alpha_{3}\right) s_{1}=\left(\alpha_{1}\right) s_{3}=\alpha_{1}+\alpha_{3} \\
& \left(\alpha_{2}\right) s_{4}=\left(\alpha_{4}\right) s_{2}=\alpha_{2}+\alpha_{4} \\
& \left(\alpha_{j}\right) s_{i}=\alpha_{i}+\alpha_{j} \text { if }|i-j|=1 \text { and }\{i, j\} \neq\{1,2\},\{2,3\} \\
& \left(\alpha_{i}\right) s_{i}=-\alpha_{i},
\end{aligned}
$$

with $\left(\alpha_{i}\right) s_{i}=\alpha_{j}$ for all other pairs $(i, j)$. As in $E_{6}(q)$ and $E_{7}(q)$ commutators are simplified as there is but one root length. We use the notation of Table 4 and distinguish the roots $r, \alpha, \beta, \gamma, \delta, \varepsilon, \varphi, \psi, \theta, \omega$. From (12.10) we have

(17.1) Each involution in $E_{8}(q), q$ even, is conjugate to one of the following

i) $x=U_{r}(1)$

ii) $y=U_{\alpha}(1) U_{\beta}(1)$

iii) $z=U_{r}(1) U_{\delta}(1) U_{s}(1)$

iv) $v=U_{\varphi}(1) U_{\psi}(1) U_{\theta}(1) U_{\omega}(1)$.

We will determine the centralizers of $x, y, z, v$. For $x=U_{r}(1)$ we use the following information from Section 4 of [7]. $C_{G}(x)=O^{2}\left(P_{8}\right)=$ $Q_{8} L_{8}$ and $L_{8} \cong E_{7}(q)$ acts irreducibly on $Q_{8} / U_{r}$. Also we check that $P_{8}=$ $Q_{8}\left(L_{8} \times W_{0}\right)$, when $W_{0}$ is cyclic of order $q-1, W_{0}$ is regular on $U_{r}^{*}$, and $C_{G}\left(W_{0}\right)=W_{0} \times L_{8}$.

Next consider $C_{G}(y), y=U_{\alpha}(1) U_{\beta}(1)$. Since $\alpha, \beta \in \mathscr{L}_{2}^{1} \quad U_{\alpha} U_{\beta} \leq Z\left(Q_{1}\right)$ and $U_{0}=Q_{1} \leq C_{U}(y)$. The roots $s \in \Delta^{+}$such that $U_{s}$ does not centralize $U_{\alpha}$ or $U_{\beta}$ are as follows

$$
\begin{gathered}
U_{\alpha} \quad\left\{s=\alpha_{2}, \alpha_{2}+\alpha_{4}, \alpha_{2}+\alpha_{4}+\alpha_{5}, \alpha_{2}+\alpha_{4}+\alpha_{5}+\alpha_{6},\right. \\
\left.\alpha_{2}+\alpha_{4}+\alpha_{5}+\alpha_{6}+\alpha_{7}, r_{6}\right\}\left\{s+\alpha=r_{52}, r_{53}, r_{54}, r_{55}, r_{56}, r_{57}\right\} \\
U_{\beta} \quad\left\{s=\alpha_{3}, \alpha_{3}+\alpha_{4}, \alpha_{3}+\alpha_{4}+\alpha_{5}, \alpha_{3}+\alpha_{4}+\alpha_{5}+\alpha_{6},\right. \\
\\
\left.\alpha_{3}+\alpha_{4}+\alpha_{5}+\alpha_{6}+\alpha_{7}, r_{7}\right\} \quad\left\{s+\beta=r_{52}, r_{53}, r_{54}, r_{55}, r_{56}, r_{57}\right\} .
\end{gathered}
$$


Consequently $C_{U}\left(U_{\alpha} U_{\beta}\right)=\left\langle U_{s}: s\right.$ not in (17.2) $\rangle$. In addition for each $c \in \boldsymbol{F}_{q}, y$ is centralized by

$$
\begin{gathered}
U_{\alpha_{3}+\alpha_{4}}(c) U_{\alpha_{2}+\alpha_{4}}(c), U_{\alpha_{3}+\alpha_{4}+\alpha_{5}}(c) U_{\alpha_{2}+\alpha_{4}+\alpha_{5}}(c), U_{\alpha_{3}+\alpha_{4}+\alpha_{5}+\alpha_{6}}(c) U_{\alpha_{2}+\alpha_{4}+\alpha_{5}+\alpha_{6}}(c) \\
U_{\alpha_{3}+\alpha_{4}+\alpha_{5}+\alpha_{6}+\alpha_{7}}(c) U_{\alpha_{2}+\alpha_{4}+\alpha_{5}+\alpha_{6}+\alpha_{7}}(c), U_{r_{7}}(c) U_{r_{6}}(c) .
\end{gathered}
$$

It now follows that $C(y)$ contains $L_{0}$ where

$$
\begin{aligned}
L_{0} & =\left\langle U_{\alpha_{2}}(c) U_{\alpha_{3}}(c), U_{\alpha_{4}}, U_{\alpha_{5}}, U_{\alpha_{8}}, U_{\alpha_{7}}, U_{\alpha_{8}}, s_{2} s_{3}, s_{4}, s_{5}, s_{6}, s_{7}, s_{8}: c \in F_{q}\right\rangle \\
& \cong B_{6}(q) \cong S p(12, q) \cong S O(13, q) .
\end{aligned}
$$

Now $U_{0}^{\prime}=U_{1}^{\prime}=Q_{2}^{1}$. As in other situations it is easy to define a nondegenerate quadratic form in $Q_{1}^{\prime}$ that is preserved by $L_{1} \cong S O^{+}(14, q)$. In this action the root groups are isotropic 1-spaces and $U_{\alpha} U_{\beta}$ is a hyperbolic plane. The element $y$ is anisotropic so $C_{L_{1}}(y) \cong S O(13, q)$. It follows that $X=U_{0} L_{0}=C_{P_{1}}(y)$. We check that $\left[L_{0}, U_{0}\right]=U_{0}$.

We claim that $P_{1}$ is the only parabolic subgroup of $G$ that contains $X$. Say $X \leq P_{i}^{q}$. By order considerations with respect to $X / U_{0} \cong S p(12, q)$ we see that $i=1,7$, or 8 . We can eliminate $i=7$ since $S p(12, q) \not E_{6}(q)$. Consequently $i=1$ or 8 and by orders and (2.3) we have $U_{0} \leq O_{2}\left(P_{i}^{q}\right)$. Then $i=8$ is out since $\left|U_{0}^{\prime}\right|>q=\left|Q_{8}^{\prime}\right|$. Consequently $i=1, U_{0}=O_{2}\left(P_{1}^{g}\right)$ $=Q_{1}^{g}$, and $g \in P_{1}$ as desired.

Since $C_{G}(y)$ is contained in some parabolic subgroup of $G$ we can now conclude that $X=C_{G}(y)$. Since $H$ is transitive on $U_{\alpha}^{\sharp} U_{\beta}^{\sharp}$ there is an element $h \in H$ such that $h$ centralizes $U_{\beta}$ but not $U_{\alpha}$. Setting $P=$ $\left\langle U_{\alpha}(c) U_{\beta}(c): c \in F_{q}\right\rangle$, we have $P P^{h}=P \times U_{\alpha}$ contains $2(q-1)$ conjugates of $x$ and $(q-1)^{2}$ conjugates of $y$. Also we check that $P=Z\left(C_{G}(y)\right)$.

Next we find $C_{G}(z)$, where $z=U_{\gamma}(1) U_{\delta}(1) U_{s}(1)$. We first note that the roots $s \in \mathscr{L}_{7}$ such that $U_{s} \not \subset C\left(U_{\gamma} U_{\delta} U_{\varepsilon}\right)$ are as follows

$$
r_{14}-\alpha_{8}, r_{15}-\alpha_{8}, r_{16}-\alpha_{8}, r_{14}, r_{15}, r_{16} \text {. }
$$

However $z$ is centralized by each of

$$
\begin{aligned}
& U_{r_{14-\alpha_{8}}}(c) U_{r_{15}-\alpha_{8}}(c), U_{r_{15}-\alpha_{8}}(c) U_{r_{16-\alpha_{8}}}(c) \\
& U_{r_{14}}(c) U_{r_{15}}(c), U_{r_{15}}(c) U_{r_{18}}(c)
\end{aligned}
$$

for $c \in F_{q}$. It follows that

(17.7) $U_{0}=\left\langle U_{t}, U_{\varphi_{i}}(c) U_{\varphi_{j}}(c): t \in \mathscr{L}_{7}, t\right.$ not in (17.9) and $U_{\varphi_{i}}(c) U_{\varphi_{j}}(c)$ as in $(17.6)\rangle=C_{Q_{7}}(z)$. 
Moreover $\left|Q_{7}: U_{0}\right|=q^{2}$ and $\left|U_{0}\right|=q^{81}$. Next let

$$
L_{00}=\left\langle U_{\alpha_{2}}, U_{\alpha_{4}}, U_{\alpha_{3}}(c) U_{\alpha_{5}}(c), U_{\alpha_{1}}(c) U_{\alpha_{8}}(c), s_{2}, s_{4}, s_{3} s_{5}, s_{1} s_{6}: c \in F_{q}\right\rangle
$$

Then using (3.1) we have $L_{00} \leq C(z)$. In addition $L_{00}$ is the fixed point group under the graph automorphism of the Levi factor, $L$, of $P_{7,8}$ we have $L_{00} \cong F_{4}(q)$ and $L_{00}$ maximal in $L$ by (16.13). Consequently

$$
L_{0}=\left\langle L_{00}, U_{ \pm \alpha_{8}}\right\rangle \cong F_{4}(q) \times S L(2, q) \quad X=U_{0} L_{0}=C_{P_{7}}(z) .
$$

Next we use Table 4 and (3.1) to show $U_{0}^{\prime}=Q_{7}^{\prime}=Q_{2}^{7}$ and $\left[U_{0}, U_{0}, U_{0}\right]=$ $Q_{3}^{7}$. Moreover $z \in U_{0}^{\prime}$ and $\left[L_{0}, U_{0}\right]=U_{0}$.

We claim that the only parabolic subgroup of $G$ containing $X$ is $P_{7}$. For suppose that $X \leq P_{i}^{q}$. Since $X$ involves $F_{4}(q) \times S L(2, q)$ the only possible values for $i$ are $i=1,2,7,8$. By (2.3) we can choose a suitable parabolic subgroup $P$ of $P_{i}^{g}$ such that $U_{0} \leq O_{2}(P)$. Then $P / O_{2}(P)$ will involve $F_{4}(q) \times S L(2, q)$. But then in $P / O_{2}(P)$ some involution contains $F_{4}(q)$ in its centralizer. Since $\left|U_{0}\right|=q^{81}$ we can easily check that only $i=7$ is possible and here $P=P_{7}^{g}$. Then $Q_{2}^{7}=\left[U_{0}, U_{0}, U_{0}\right]=\left(Q_{2}^{7}\right)^{g}$ and $g \in N_{G}\left(Q_{2}^{7}\right)=P_{7}$. This proves the claim. Since $C_{G}(z)$ is contained in a parabolic subgroup we now have $X=C_{G}(z)$.

Set $P=\left\langle U_{r}(c) U_{\delta}(c) U_{\varepsilon}(c): c \in F_{q}\right\rangle$. Then $P=Z\left(C_{G}(z)\right)$ and there is an element $h \in H$ centralizing $U_{\delta} U_{s}$ but not $U_{r}$. Consequently $P P^{h}=U_{r} \times P$ contains $q-1$ conjugates of $x, q-1$ conjugates of $y$, and $(q-1)^{2}$ conjugates of $z$. We check that $P=Z(X)$.

We still must find $C_{G}(u)$, where $u=U_{\varphi}(1) U_{\psi}(1) U_{\theta}(1) U_{\omega}(1)$. We begin with $C_{Q_{2}}(u)$. Listed below are the roots $s \in \mathscr{L}_{2}$ such that $U_{s}$ does not centralize one of the subgroups $U_{\varphi}, U_{\psi}, U_{\theta}, U_{\omega}$.

$$
\begin{aligned}
U_{\varphi}\{s= & \left.r_{6}, r_{8}, r_{11}, r_{13}, r_{16}, r_{31}\right\}\left\{s+\varphi=r_{50}, r_{52}, r_{53}, r_{54}, r_{55}, r_{56}\right\} \\
U_{\psi}\{s= & \left.r_{6}-\alpha_{8}, r_{10}-\alpha_{8}, r_{12}-\alpha_{8}, r_{14}-\alpha_{8}, r_{18}-\alpha_{8}, r_{32}\right\} \\
& \left\{s+\psi=r_{48}, r_{52}, r_{53}, r_{54}, r_{55}, r_{57}\right\} \\
U_{\theta}\{s= & \alpha_{2}+\alpha_{3}+2 \alpha_{4}+\alpha_{5}, r_{12}-\alpha_{6}-\alpha_{7}-\alpha_{8}, \\
& \left.r_{15}-\alpha_{6}-\alpha_{7}-\alpha_{8}, r_{20}-\alpha_{7}-\alpha_{8}, r_{20}-\alpha_{8}, r_{20}\right\} \\
& \left\{s+\theta=r_{48}, r_{50}, r_{52}, r_{55}, r_{56}, r_{57}\right\} \\
U_{\omega}\{s= & r_{8}-\alpha_{7}-\alpha_{8}, r_{10}-\alpha_{7}-\alpha_{8}, r_{15}-\alpha_{7}-\alpha_{8}, \\
& \left.r_{17}-\alpha_{7}-\alpha_{8}, r_{19}-\alpha_{8}, r_{19}\right\} \\
& \left\{s+\omega=r_{48}, r_{50}, r_{53}, r_{54}, r_{56}, r_{57}\right\} .
\end{aligned}
$$


Consequently $C_{Q_{2}}\left(U_{\varphi} U_{\psi} U_{\theta} U_{\omega}\right)=\left\langle U_{s}: s \in \mathscr{L}_{2}, s\right.$ not in (17.10) $\rangle$. In addition for each $c \in F_{q} u$ is centralized by each element of the form

$$
U_{\gamma_{1}}(c) U_{\gamma_{2}}(c),
$$

where $\gamma_{1}, \gamma_{2}$ appear in (17.10) and $\gamma_{1}+\rho_{1}=\gamma_{2}+\rho_{2}$ for $\rho_{1}, \rho_{2} \in\{\varphi, \psi, \theta, \omega\}$. Consequently we have

$$
U_{0}=C_{Q_{2}}(u)=\left\langle U_{t}, U_{\gamma_{1}}(c) U_{r_{2}}(c): t \in \mathscr{L}_{2}, t \text { not in (17.10), } \gamma_{1}, \gamma_{2}\right. \text { as in }
$$

Next we set

$$
L_{0}=\left\langle U_{\alpha_{5}}, U_{\alpha_{1}}(c) U_{\alpha_{8}}(c), U_{\alpha_{3}}(c) U_{\alpha_{7}}(c), U_{\alpha_{4}}(c) U_{\alpha_{8}}(c), s_{5}, s_{1} s_{8}, s_{3} s_{7}, s_{4} s_{6}: c \epsilon\right.
$$
$F_{q}>$.

Then we check that $L_{0} \leq C(u)$. Moreover if $L$ is the Levi factor of $P_{2}$ then $L_{0}$ is the fixed point group under the graph automorphism. Consequently $L_{0} \cong S p(8, q)$. By (16.8) $L_{0}$ is maximal in $L$, and since $L \not \subset C(u)$ we check the action of $H$ and conclude that $X=U_{0} L_{0}=C_{P_{2}}(u)$.

We note that $U_{0} \leq Q_{2}$ and $\left|U_{0}\right|=q^{82}$. Using Table 3 and 4 we check that $U_{0}^{\prime}=Q_{2}^{\prime}=Q_{2}^{2}$ and that $\left[U_{0}, U_{0}, U_{0}\right]=\left[Q_{2}, Q_{2}, Q_{2}\right]=Q_{3}^{2}$. Then $u \in U_{0}^{\prime}$. Also $\left|U_{0}^{\prime}\right|=q^{36}$ and $\left|\left[U_{0}, U_{0}, U_{0}\right]\right|=q^{8}$. We have $L_{2}$ acting on $\left[U_{0}, U_{0}, U_{0}\right]$ as on the natural module for $S L(8, q)$ and there is a non-degenerate alternating form preserved by $L_{0}$. Also $\left[L_{0}, X_{0}\right]=X_{0}$ and we can use the action of $L_{0}$ on $U_{0}$ and the structure of $U_{0}$ to see that $Z(X)=$ $\left\langle U_{\varphi}(c) U_{\psi}(c) U_{\theta}(c) U_{\omega}(c): c \in \boldsymbol{F}_{q}\right\rangle$.

We claim that $P_{2}$ is the only parabolic subgroup of $G$ containing $X$. Say $X \leq P_{i}^{q}=P$. Then $L_{0} \cong S p(8, q)$ implies that $i \neq 3,4,5$. First suppose that $i=8$. Then $\left|O_{2}(P)\right|=\left|Q_{8}\right|=q^{57}$ and $P^{\prime}=C_{G}\left(Z\left(O_{2}(P)\right)\right)$. If $Z\left(O_{2}(P)\right)$ $\cap X \neq 1$ then $Z\left(O_{2}(P)\right) \cap X$ is properly contained in $Z(X)$.

We first claim that $Z\left(O_{2}(P)\right) \leq U_{0}$. Otherwise since $O_{2}(P)$ is special with center $U_{r}^{g}$ we have $\left(U_{0} \cap O_{2}(P)\right) /\left(U_{0} \cap Z\left(O_{2}(P)\right)\right)$ abelian and with the structure of $O_{2}(P) / U_{0} \cap Z\left(O_{2}(P)\right)$ this forces $\left|U_{0} \cap O_{2}(P)\right|<q^{29}$, and hence $\left|U_{0} O_{2}(P) / O_{2}(P)\right|>q^{53}$. But since $P^{\prime} / O_{2}(P) \cong E_{7}(q)$ and $L_{0} \cong S p(8, q)$ this is impossible. Consequently $Z\left(O_{2}(P)\right) \leq U_{0}$.

Now $\left|U_{0} O_{2}(P) / O_{2}(P)\right| \geq q^{82-57}=q^{25}$ and $U_{0}^{\prime}$ has order $q^{36}$ and is abelian. Again the structure of $O_{2}(P)$ forces $\left|U_{0}^{\prime} \cap O_{2}(P)\right| \leq q^{29}$. As $Z\left(O_{2}(P)\right) \leq Z(X)$, $Z(X)=U_{r}^{g}=Z\left(O_{2}(P)\right.$ ) (which would give $\left.u \sim x\right)$. Considering the possibilities for $U_{0} O_{2}(P) / O_{2}(P)$ we see that $\left|U_{0}^{\prime} \cap O_{2}(P)\right|>q$. From here it 
follows that $\left[U_{0}, U_{0}, U_{0}\right]=Q_{3}^{2} \leq O_{2}(P)$. Indeed $L_{0}$ acts irreducibly on $\left[U_{0}, U_{0}, U_{0}\right]$ so if the containment were false then $\left[U_{0}, U_{0}, U_{0}\right] \cap O_{2}(P)=1$. But no parabolic subgroup $D$ of $E_{7}(q)$ involving $S p(8, q)$ has $O_{2}(D)$ of class at least 3 with $\left|\left[O_{2}(D), O_{2}(D), O_{2}(D)\right]\right| \geq q^{8}$. The assertion follows. Let $\left|U_{0}^{\prime} \cap U_{2}(P)\right|=q^{\ell}$. Then $9 \leq \ell \leq 29$ by the above. Since $Z(X) \cap$ $\left[U_{0}, U_{0}, U_{0}\right]=1, U_{0} \cap O_{2}(P)$ must centralize $U_{0}^{\prime} \cap O_{2}(P)$. The structure of $O_{2}(P)$ then implies that $\left|U_{0} \cap O_{2}(P)\right| \leq q^{57-\ell}$ and $\left|U_{0} O_{2}(P) / O_{2}(P)\right| \geq q^{25+\ell} \geq q^{34}$. Next we use the fact that $Q_{3}^{2} \leq O_{2}(P)$ and $Q_{3}^{2}$ is abelian to get $C_{O_{2}(P)}\left(Q_{3}^{2}\right)$ $=q^{49}$ (here we use the theory ofextra special groups). But $C_{O_{2}(P)}\left(Q_{3}^{2}\right) \leq$ $N_{G}\left(Q_{3}^{2}\right)=P_{2}$, and this implies that $C_{O_{2}(P)}\left(Q_{3}^{2}\right) \leq U_{0}$ and $\left|U_{0} \cap O_{2}(P)\right| \geq q^{49}$. But then $\left|U_{0}\right| \geq q^{49} q^{34}=q^{83}$, a contradiction. Thus $i \neq 8$.

Suppose that $i=7$. Then $\left|O_{2}(P)\right|=q^{83}$. Since $\left|\left[Q_{7}, Q_{7}, Q_{7}\right]\right|=\left|Q_{3}^{7}\right|=q^{2}$, we cannot have $U_{0} \leq O_{2}(P) \cong Q_{7}$. The only possibility is $X \leq P_{i}^{q} \cap P_{j}^{q p}$ for $j=1$ or 6 and $g \in P$. Then $\left|U_{0} O_{2}(P) / O_{2}(P)\right| \leq q^{16}$ and $\left|U_{0} \cap U_{2}(P)\right| \geq q^{66}$. Also in this case $U_{0}^{\prime} \leq O_{2}(P)$, so $Q_{3}^{2} \leq O_{2}(P)$ and $Z\left(O_{2}(P)\right) \leq N\left(Q_{3}^{2}\right)=P_{2}$. But $O_{2}(P)$ has center of order $q^{2}$ which will be centralized by $L_{0}$. This contradicts $|Z(X)|=q$.

Next assume that $i=6$. Here $\left(P / O_{2}(P)\right)^{\prime} \cong S O^{+}(10, q) \times S L(3, q)$. As $\left[L_{0}, U_{0}\right]=U_{0}$ we must have $U_{0} \leq O_{2}(P)$. Since $O_{2}(P) \sim Q_{6}, Z\left(O_{2}(P)\right) \sim Q_{4}^{6}$ which is centralized by $L_{0}$. As above this contradicts $|Z(X)|=q$.

If $i=1$, then $\left|O_{2}(P)\right|<\left|U_{0}\right|$ and we have $U_{0} \not \leq O_{2}(P)$. Then we have $X \leq P_{1}^{g} \cap P_{j}^{g p}$ for some $j$ and some $g \in P$. By orders $j \neq 2$, so by our previous work this is impossible.

Finally if $i=2$ we argue that by the above $U_{0} \leq O_{2}(P)$ and then $Q_{3}^{2}=\left[U_{0}, U_{0}, U_{0}\right]=\left[O_{2}(P), O_{2}(P), O_{2}(P)\right]=\left(Q_{3}^{2}\right)^{g}$. So $g \in N\left(Q_{3}^{2}\right)=P_{2}$. This proves the claim. Consequently $C_{G}(u)=C_{P_{2}}(u)=X$.

Setting $P=\left\langle U_{\varphi}(c) U_{\psi}(c) U_{\theta}(c) U_{\omega}(c): c \in F_{q}\right\rangle$ we have $P=Z(X)$ and there is an element $h \in H$ centralizing $U_{\psi} U_{\theta} U_{\omega}$ but not $U_{\varphi}$. Then $P P^{h}=$ $P \times U_{\varphi}$ contains $q-1$ conjugates of $z$, and $(q-1)^{2}$ conjugates of $u$.

We have now completed our analysis of $E_{8}(q)$ and we summarize our results as follows.

(17.14) The maximal parabolic subgroups of $G$ containing the centralizers of the involutions in (17.1) are as follows.
i) $C_{G}(x) \leq P_{8}$
ii) $C_{G}(y) \leq P_{1}$
iii) $C_{G}(z) \leq P_{7}$ 
iv) $C_{G}(u) \leq P_{2}$.

In particular no two of the involutions $x, y, z, u$ are conjugate.

(17.15) i) $C_{G}(x)=O^{2^{\prime}}\left(P_{8}\right)=Q_{8} L_{8}$, where $L_{8} \cong E_{7}(q)$ acts irreducibly on $Q_{8} / U_{r}=Q_{8} / Q_{8}^{\prime}$. Also $P_{8}=Q_{8}\left(L_{8} \times W_{0}\right)$ where $W_{0}$ is cyclic of order $q-1$ and $C_{G}\left(W_{0}\right)=W_{0} \times L_{8}$.

ii) $C_{G}(y)=U_{0} L_{0}$, where $U_{0}=Q_{1}$ and $L_{0} \cong S p(12, q)$ is as in (17.4). Also $y \in U_{0}^{\prime}=Z\left(U_{0}\right),\left[L_{0}, U_{0}\right]=U_{0}$, and $C_{G}(y)^{\prime}=C_{G}(y)$. Let $P=\left\langle U_{\alpha}(c)\right.$ $\left.U_{\beta}(c): c \in \boldsymbol{F}_{q}\right\rangle$. Then $P=Z\left(C_{G}(y)\right)$ and there is an element $h \in H$ such that $P P^{h}=P \times U_{\alpha} \leq C_{G}(y)$ contains $2(q-1)$ conjugates of $x$ and $(q-1)^{2}$ conjugates of $y$. The group $L_{1} \cong S O^{+}(14, q)$ acts on $U_{0}^{\prime}$ preserving a non-degenerate quadratic form. In this action $P$ is an anisotropic 1space.

iii) $C_{G}(z)=U_{0} L_{0}$ where $U_{0} \leq Q_{7}$ is given by (17.8) and $L_{0} \cong F_{4}(q)$ $\times S L(2, q)$ is given by (17.9). We have $z \in U_{0}^{\prime}=Q_{2}^{7}$ and $\left[U_{0}, U_{0}, U_{0}\right]=Q_{3}^{7}$. Also $P=\left\langle U_{r}(c) U_{\theta}(c) U_{\varepsilon}(c): c \in F_{q}\right\rangle=Z\left(C_{G}(Z)\right),\left[L_{0}, U_{0}\right]=U_{0}$ and $\left|C_{G}(y): C_{G}(y)^{\prime}\right|$ $=1,2$ depending on whether $q>2$ or $q=2$. There is an element $h \in H$ such that $P P^{h}=P \times U_{r} \leq C_{G}(z)$ contains $q-1$ conjugates of $x, q-1$ conjugates of $y$, and $(q-1)^{2}$ conjugates of $z$.

iv) $C_{G}(u)=U_{0} L_{0}$, where $U_{0}, L_{0}$ are given in (17.12) and (17.13). Here $U_{0} \leq Q_{2}$ and $L_{0} \cong S p(8, q)$. We have $u \in U_{0}^{\prime}=Q_{2}^{\prime},\left[U_{0}, U_{0}, U_{0}\right]=Q_{3}^{2}$, $\left[L_{0}, U_{0}\right]=U_{0}$, and $C_{G}(y)=C_{G}(y)^{\prime}$. Setting $P=\left\langle U_{\varphi}(c) U_{\psi}(c) U_{\theta}(c) U_{\omega}(c)\right.$ : $\left.c \in \boldsymbol{F}_{q}\right\rangle$ we have $P=Z\left(C_{G}(u)\right)$. There is an element $h \in H$ such that $P P^{h}=P \times U_{\varphi} \leq C_{G}(u)$ contains $q-1$ conjugates of $x, q-1$ conjugates of $z$, and $(q-1)^{2}$ conjugates of $u$.

\section{Section 18. Involutions and centralizers in the exceptional rank 2 groups.}

In this section let $G=G_{2}(q) q>2,{ }^{3} D_{4}(q),{ }^{2} F_{4}(q)$ or ${ }^{2} F_{4}(2){ }^{\prime}$, where $q$ is a power of 2 . We will simply record the information on centralizers since much of this already appears in the papers of Thomas [23], Thomas [24], and Parrot [18]. For example the involution classes are given in these papers. The centralizers are either explicitly presented in the appropriate reference or easily obtained from the given information and the commutator relations. These results are also easily obtained from Fong-Seitz [10] Sections 9 and 10, using a root diagram and arguing as in earlier sections.

In each case the group $G$ has a $(B, N)$-pair of rank 2 (except for 
$\left.{ }^{2} F_{4}(2)^{\prime}\right)$ and we can label the roots with respect to a fundamental set $\left\{\alpha_{1}, \alpha_{2}\right\}$ for $\Delta$. Then $\left\langle s_{1}, s_{2}\right\rangle \cong D_{12}$ if $G=G_{2}(q)$ or ${ }^{3} D_{4}(q)$ and $\left\langle s_{1}, s_{2}\right\rangle \cong D_{16}$ if $G \cong{ }^{2} F_{4}(q)$. In the first case we assume that $\alpha_{1}$ is a long root and $\alpha_{2}$ a short root.

Listing the roots in $\Delta^{+}$we have

$$
\begin{aligned}
\Delta^{+}= & \left\{r_{1}=\alpha_{1}, r_{2}=\alpha_{2}, r_{3}=\left(\alpha_{1}\right) s_{2}, r_{4}=\left(\alpha_{2}\right) s_{1}, r_{5}\right. \\
& \left.=\left(\alpha_{1}\right) s_{2} s_{1}, r_{6}=\left(\alpha_{2}\right) s_{1} s_{2}\right\}, \text { or } \\
\Delta^{+}= & \left\{r_{1}=\alpha_{1}, r_{2}=\alpha_{2}, r_{3}=\left(\alpha_{1}\right) s_{2}, r_{4}=\left(\alpha_{2}\right) s_{1}, r_{5}\right. \\
= & \left.\left(\alpha_{1}\right) s_{2} s_{1}, r_{6}=\left(\alpha_{2}\right) s_{1} s_{2}, r_{7}=\left(\alpha_{1}\right) s_{2} s_{1} s_{2}, r_{8}=\left(\alpha_{2}\right) s_{1} s_{2} s_{1}\right\},
\end{aligned}
$$

depending on whether $\left\langle s_{1}, s_{2}\right\rangle \cong D_{12}$ or $D_{16}$. If $G=G_{2}(q)$ then $U_{r_{1}}$ and $U_{r_{2}}$ are elementary of order $q$, while if $G={ }^{3} D_{4}(q), U_{r_{1}}$ and $U_{r_{2}}$ are elementary of order $q$ and $q^{3}$ respectively. If $G={ }^{2} F_{4}(q)$ then $q$ is an odd power of 2, and we order $\alpha_{1}, \alpha_{2}$ so that $U_{r_{1}}$ is elementary of order $q$ and $U_{r_{2}}$ is isomorphic to the Sylow 2-subgroup of $S z(q)$.

(18.2) $G$ has 2 conjugacy classes of involutions with representatives $z$, t. If $G=G_{2}(q)$ or ${ }^{3} D_{4}(q)$ we may choose $z=U_{r_{5}}(1)$ and $t=U_{r_{8}}(1)$. If $G={ }^{2} F_{4}(q)$ or ${ }^{2} F_{4}(2)^{\prime}$ we may choose $z \in \Omega_{1}\left(U_{r_{8}}\right)^{\#}$ and $t=U_{r_{7}}(1)$. $z$ is a 2-central involution and $t$ is not a 2-central involution.

Let $P_{1}=\left\langle B, s_{2}\right\rangle$ and $P_{2}=\left\langle B, s_{1}\right\rangle$ be the proper parabolic subgroups of $G$ if $G \neq{ }^{2} F_{4}(2)^{\prime}$, and if $G={ }^{2} F_{4}(2)^{\prime}$ let $P_{i}$ be the intersection with $G$ of the appropriate parabolic subgroup $\bar{P}_{i}$ of $\bar{G}={ }^{2} F_{4}(2)$.

(18.3) If $G \neq{ }^{2} F_{4}(2)^{\prime}$ then $P_{1}$ is the unique proper parabolic subgroup $G$ containing $C_{G}(z)$ and $P_{2}$ is the unique proper parabolic subgroup of $G$ containing $C_{G}(t)$.

(18.4) Let $G=G_{2}(q), q \geq 4$.

i) $C_{G}(z)=O^{2^{\prime}}\left(P_{1}\right)=U_{0} L_{0}$, where $U_{0}=O_{2}\left(P_{1}\right)$ and $L_{0}=\left\langle U_{ \pm r_{2}}\right\rangle \cong$ $S L(2, q)$. Also $Z\left(C_{G}(z)\right)=U_{r_{5}}$ and $C_{G}(z)^{\prime}=C_{G}(z)$.

ii) $C_{G}(t)=U_{0} L_{0}$, where $U_{0}=U_{r_{3}} \times U_{r_{5}} \times U_{r_{8}}$ and $L_{0}=\left\langle U_{ \pm r_{1}}\right\rangle \cong$ $S L(2, q)$. Also $U_{0} L_{0}=\left(U_{r_{3}} \times U_{r_{5}}\right) L_{0} \times U_{r_{6}}$ where $L_{0}$ acts irreducibly on $U_{r_{3}} \times U_{r_{5}}$.

(18.5) Let $G={ }^{3} D_{4}(q)$

i) $C_{G}(z)=O^{2^{\prime}}\left(P_{1}\right)=U_{0} L_{0}$, where $U_{0}=O_{2}\left(P_{1}\right)$ and $L_{0}=\left\langle U_{ \pm r_{2}}\right\rangle \cong$ $S L\left(2, q^{3}\right) . \quad U_{0}^{\prime} \geq Z\left(C_{G}(z)\right)=U_{r_{5}}$ and $C_{G}(z)^{\prime}=C_{G}(z)$. 
ii) $C_{G}(t)=U_{0} L_{0}$, where $L_{0}=\left\langle U_{ \pm r_{1}}\right\rangle \cong S L(2, q)$ and $U_{0}=U_{r_{3}} U_{r_{6}} U_{r_{5}} V_{r_{2}}$ $V_{r_{4}}$ where $V_{r_{i}}=\left\{U_{r_{i}}(c): c \in \boldsymbol{F}_{q^{3}}\right.$ and $\left.\operatorname{tr}(c)=0\right\}$. Also $\left[L_{0}, U_{0}\right]=U_{0}$ and $U_{0}^{\prime} \geq Z\left(C_{G}(t)\right)=\left\{U_{r_{6}}(c): c \in \boldsymbol{F}_{q}\right\}$.

(18.6) Let $G={ }^{2} F_{4}(q)$. If $q=2$, then ${ }^{2} F_{4}(q)-{ }^{2} F_{4}(q)^{\prime}$ contains no involutions.

i) $C_{G}(z)=O^{2^{\prime}}\left(P_{1}\right)=U_{0} L_{0}$, where $U_{0}=O_{2}\left(P_{1}\right)$ and $L_{0}=\left\langle U_{ \pm r_{2}}\right\rangle \cong$ $S z(q) . \quad Z\left(C_{G}(z)\right)=\Omega_{1}\left(U_{r_{8}}\right)$ and $\left[U_{0}, L_{0}\right] U_{r_{8}}=U_{0}$. Also $\Omega_{1}\left(U_{r_{8}}\right) \leq\left[U_{0}, L_{0}\right]^{\prime}$.

ii) $C_{G}(t)=U_{0} L_{0}$, where $L_{0}=\left\langle U_{ \pm r_{1}}\right\rangle \cong S L(2, q)$ and $U_{0}=\Omega_{1}\left(U_{r_{2}}\right)$ $U_{r_{3}} \Omega_{1}\left(U_{r_{4}}\right) U_{r_{5}} U_{r_{6}} U_{r_{7}} U_{r_{8}}$. Here $U_{0}^{\prime}=\Omega_{1}\left(U_{r_{6}}\right) U_{r_{7}} \Omega_{1}\left(U_{r_{8}}\right), Z\left(C_{G}(t)\right)=U_{r_{7}}$, and $\left[U_{0}, L_{0}\right]=U_{0}$.

Let $U_{0} L_{0}$ be the centralizer of an involution as described in (18.4), (18.5) or (18.6). Assume that $L_{0} \not S_{3}$ or $S z(2)$. Let $W_{0}=L_{0} \cap H$, except in case $G={ }^{3} D_{4}(q)$ and $U_{0} L_{0}=C_{G}(z)$, in which case we set $W_{0}=$ $\left(L_{0} \cap H\right)^{q-1}$, a cyclic group of order $q^{2}+q+1$. Then

(18.7) $E\left(C_{G}\left(W_{0}\right)\right)=O^{2^{\prime}}\left(C_{G}\left(W_{0}\right)\right)=L$ with $L$ as follows

i) If $G=G_{2}(q)$ and $C_{G}(z)=U_{0} L_{0}$, then $L=\left\langle U_{ \pm r_{5}}\right\rangle \cong S L(2, q)$ if. $q>4$, and $L=\left\langle U_{ \pm r_{1}}, U_{ \pm r_{3}}, U_{ \pm r_{5}}\right\rangle \cong S L(3,4)$ if $q=4$.

ii) If $G={ }^{3} D_{4}(q)$ and $C_{G}(z)=U_{0} L_{0}$, then $L=\left\langle U_{ \pm r_{1}}, U_{ \pm r_{3}}, U_{ \pm r_{5}}\right\rangle \cong$ $\operatorname{PSL}(3, q)$.

iii) If $C={ }^{2} F_{4}(q)$ and $C_{G}(z)=U_{0} L_{0}$, then $L=\left\langle U_{ \pm r_{8}}\right\rangle \cong S z(q)$.

iv) If $G=G_{2}(q)$ or ${ }^{3} D_{4}(q)$ and $C_{G}(t)=U_{0} L_{0}$, then $L=\left\langle U_{ \pm r_{6}}\right\rangle \cong$ $S L(2, q)$ or $S L\left(2, q^{3}\right)$, respectively.

v) If $G={ }^{2} F_{4}(q)$ and $C_{G}(t)=U_{0} L_{0}$, then $L=\left\langle U_{ \pm r_{7}}\right\rangle \cong S L(2, q)$.

\section{Section 19. Outer automorphisms.}

Let $G=G(q)$ be a Chevalley group of characteristic 2 with $Z(G)=1$ having root system $\Delta$, and regard $G \unlhd \operatorname{Aut}(G)$. In this section we will determine the classes of involutions in $\operatorname{Aut}(G)-G$ and their centralizers in $G$.

First we need the involutions in Out $(G)$. In any case Out $(G)$ has at most 3 classes of involutions. If $\Delta$ has no double bonds and $G$ is untwisted, then each involution in $\operatorname{Out}(G)$ is conjugate to a graph, field, or graph-field automorphism of $G$. In this case Out $(G)$ can have 0,1 , or 3 classes of involutions depending on the existence of field automorphisms of $\boldsymbol{F}_{q}$ and on whether $\Delta$ admits graph automorphisms. When $G$ 
is untwisted and $\Delta$ has a double bond and admits a graph automorphism ( $\Delta=F_{4}$ or $B_{2}$, as $G_{2}$ will not occur in even characteristic) a different situation occurs. For this see (19.3). In the twisted groups there will be a unique involution in Out $(G)$ except for $G={ }^{2} F_{4}(q)$ where there is none and $G={ }^{3} D_{4}(q)$ where there is one only if $q$ is a square.

If $G=P S O^{-}(2 n, q)$, then Out $(G)$ has just one involution and the results of Section 8 give the necessary information on involutions in Aut $(G)-G$. For $G=P S O^{+}(2 n, q)$ Section 8 gives the involutions in the coset of the graph automorphism of $\Delta$. Consequently these cases will not be discussed here.

The first result handles field automorphisms.

(19.1) Suppose that $G=G(q)$ is an untwisted Chevalley group or that $G \cong{ }^{3} D_{4}(q)$. Assume that $q=q_{0}^{2}$ and that $\sigma$ is an involutory field automorphism of $G$. Then Go contains just one class of involutions and we have $C_{G}(\sigma) \cong G\left(q_{0}\right)$.

Proof. This follows from Lang's theorem [17]. However an elementary proof is as follows. Let $B=U H$ be a Borel group normalized by $\sigma$, chosen such that $H$ is a $\sigma$-invariant abelian group of odd order. Write $H=H_{0} \times H_{1}$, where $H_{0}=C_{H}(\sigma)$ and $H_{1}=[H, \sigma]$.

Let $x \in G \sigma$ be an involution. As $U\langle\sigma\rangle$ is Sylow in $G\langle\sigma\rangle$ we may assume $x \in U \sigma$. Consider the group $U H_{1}\langle\sigma\rangle$. We claim that $C_{U}\left(H_{1}\right)=1$. Otherwise considering the decomposition of $U$ into a product of root groups we see that $H_{1} \leq C\left(U_{s}\right)$ for some $s \in \Delta^{+}$. However $\sigma$ acts as a field automorphism on $\left\langle U_{ \pm s}\right\rangle \cong S L(2, q)$ (or possibly $S L\left(2, q^{3}\right)$ if $G \cong{ }^{3} D_{4}(q)$ ), so that $H_{1} \cap\left\langle U_{ \pm s}\right\rangle \neq 1$ and acts fixed-point-freely on $U_{s}$. This is impossible, proving the claim.

Now (2.1) applies and gives the result.

(19.2) The following groups $G$ have each involution in Aut $(G)-G$ conjugate to a field automorphism: $G_{2}(q),{ }^{3} D_{4}(q), P S p(2 n, q) n \geq 3, E_{7}(q)$, and $E_{8}(q)$.

Proof. For each of these groups there are no graph automorphisms and all diagonal automorphisms have odd order. The result follows from (19.1) and Steinberg's theorem [21].

(19.3) Let $G=P \operatorname{Sp}(4, q)$ or $F_{4}(q)$. Then Out $(G)$ is cyclic of order $2 a$, where $q=2^{a}$. In particular if $q$ is a square, then each involution in 
Aut $(G)-G$ is conjugate to a field automorphism of $G$.

Proof. If $\sigma$ is a graph automorphism of $G$ then one checks (see Carter. [6], Section 12.3) that $\sigma$ can be chosen such that $\left\langle\sigma^{2}\right\rangle$ is the group of field automorphisms of $G$. Consequently Out $(G)=\langle\sigma\rangle$. If $\sigma^{2}$ has even order, then each involution in Out $(G)$ is in $\left\langle\sigma^{2}\right\rangle$ and the result follows from (19.1). This proves (19.3).

The following lemma is easily checked.

(19.4) Let $\tau$ be an involution acting on an elementary 2-group $M$. If $\left|C_{M}(\tau)\right|=|M|^{1 / 2}$, then each involution in $M \tau$ is conjugate to $\tau$.

(19.5) Suppose that $G=P S p(4, q)$ or $F_{4}(q)$ and let $t$ be an involution in Aut $(G)-G$. Then all involutions in Aut $(G)-G$ are conjugate. Moreover

i) $C_{G}(t) \cong P S p\left(4, q_{0}\right)$ or $F_{4}\left(q_{0}\right)$ if $q$ is a square and $q=q_{0}^{2}$.

ii) $C_{G}(t) \cong S z(q)$ or ${ }^{2} F_{4}(q)$ if $q$ is not a square.

Proof. If $q$ is a square we are done by (19.3) and (19.1). So assume that $q$ is not a square. Let $\sigma$ generate the group of graph automorphisms preserving $\Delta$ and normalizing $U$. Let $\tau$ be the involution in $\langle\sigma\rangle$. We may assume that $t \in \tau G$. In fact since $U \in \operatorname{Syl}_{2}(G)$ we may assume that $t \in \tau U$.

We will give the proof for $F_{4}(q)$, leaving the easier case of $P S p(4, q)$ to the reader. We define normal subgroups $U_{1}<U_{2}<U_{3}$ of $U$. For each $i, U_{i}$ will be the product of root groups and these roots occur in pairs, orbits under the graph automorphism of $\Delta$. The roots are

$$
\begin{gathered}
\theta_{1}=\{\{1122,1111\},\{1222,1121\},\{1242,1221\},\{1342,1231\},\{2342,1232\}\} \\
\theta_{2}=\theta_{1} \cup\{\{1120,0111\},\{1220,0121\},\{0122,1100\}\} \\
\theta_{3}=\theta_{2} \cup\{\{1100,0011\},\{0120,0110\}\}
\end{gathered}
$$

Then $U_{i}=\prod U_{s}$, where $\left\{s, s^{\prime}\right\} \in \theta_{i}$ for some $s^{\prime}$. Using (3.1) and Table 1 we easily check that each $U_{i} \unlhd U$. Let $U_{0}=1, U_{4}=U$, and consider the quotient $U_{i+1} / U_{i}=V_{i}, i=0,1,2,3$. For each such $i, V_{i}$ is the direct sum of elementary subgroups and these subgroups occur in pairs such that $\tau$ interchanges the subgroups in a given pair. Then by (19.4) all involutions in $V_{i} \tau$ are conjugate. Apply this to $V_{3}, V_{2}, V_{1}$, and $V_{0}$ and obtain $t \sim \tau$.

Finally (ii) holds since we know that some involution in $\operatorname{Aut}(G)-G$ 
has the correct centralizer.

(19.6) Let $G(q)$ be an untwisted Chevalley group admitting an involutory graph automorphism $\tau$ and an involutory field automorphism $\sigma$ such

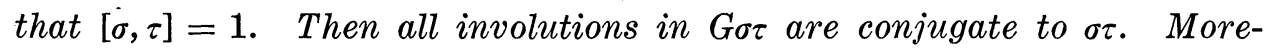
over $q=q_{0}^{2}$ and one of the following holds:

i) $G=\operatorname{PSL}(n, q)$ and $C_{G}(\sigma \tau)^{\prime} \cong \operatorname{PSU}\left(n, q_{0}\right)$

ii) $G=P S O^{+}(2 n, q)^{\prime}$ and $C_{G}(\sigma \tau)^{\prime} \cong P S O^{-}\left(2 n, q_{0}\right)^{\prime}$

iii) $G=E_{6}(q)$ and $C_{G}(\sigma \tau)^{\prime} \cong{ }^{2} E_{6}\left(q_{0}\right)$.

Proof. The information on centralizers is standard, so we need only prove that all involutions in $G \sigma \tau$ are conjugate. We argue as in (19.1). Let $B=U H$ be a Borel group. We may assume that $\sigma$ and $\tau$ normalize $U$ and $H$, and we write $H=H_{0} \times H_{1}$ with $H_{0}=C_{H}(\sigma \tau)$ and $H_{1}=[H, \sigma \tau]$. As in (19.1) it will suffice to show that $C_{U}\left(H_{1}\right)=1$.

Let $G_{0} / G$ be the group of diagonal automorphisms in Out $(G)$. Then $G_{0} / G$ is cyclic and $G_{0}=\left[G_{0}, \tau \sigma\right] C_{G_{0}}(\tau \sigma)$, so all fusion in $G_{0} \tau \sigma$ is accomplished under the action of $G$. Hence we may take $G=G_{0}$.

Let $\langle\zeta\rangle=F_{q}^{*}$ and for each $i=1, \cdots, n$ let $\chi_{i}$ be the character such that $\chi_{i}\left(\alpha_{i}\right)=\zeta$ and $\chi_{i}\left(\alpha_{j}\right)=1$ for all $j \neq i$. Set $h_{i}=h\left(\chi_{i}\right)$. Then for $i=1, \cdots, n\left[h_{i}, \sigma \tau\right] \in H_{1}$.

If $C_{U}\left(H_{1}\right) \neq 1$, then $U_{r} \leq C_{U}\left(H_{1}\right)$ for some $r \in \Delta^{+}$. Write $r=\sum \gamma_{i} \alpha_{i}$. Then

$$
\begin{aligned}
U_{r}(1)^{\left[h_{i}, \sigma \tau\right]} & =\left(U_{r}\left(\left(\zeta^{\gamma_{i}}\right)^{-1}\right)\right)^{h_{i}^{\sigma \tau}} \\
& =U_{r}\left(\zeta^{-\gamma_{i}}\right)^{h_{i}^{\sigma \tau}} \\
& =U_{r}\left(\zeta^{\delta_{i} q_{0}-\gamma_{i}}\right)
\end{aligned}
$$

where $U_{r}^{\tau}=U_{s}$ and $s=\sum \delta_{i} \alpha_{i}$. Therefore $q_{0}^{2}-1$ divides $\delta_{i} q_{0}-\gamma_{i}$. We now use information concerning the roots in $\Delta^{+}$, for which the reference is Bourbaki [5]. Since $G=P S L(n+1, q), P S O^{+}(2 n, q)$, or $E_{6}(q)$, some $\delta_{i}$ must equal 1 . Then for this value of $i$ the divisibility condition forces $\gamma_{i}-q_{0} \geq q_{0}^{2}-1$ and hence $\gamma_{i} \geq 5$. But this does not occur, proving (19.6).

The remaining cases not treated in Section 8 are $G=P S L(n, q)$, $E_{6}(q), P S U(n, q)$, and ${ }^{2} E_{6}(q)$. In the first two cases let $\sigma$ be an involutory graph automorphism preserving $\Delta$ and normalizing $U, H$ in a Borel group $B \leq G$. In the second two cases we let $\sigma$ be the graph automorphism 
of the non-twisted group defining $G$ and restrict $\sigma$ to $G$. Again we assume $B \leq G$ is Borel and normalized by $\sigma$.

We must determine the involutions in $G \sigma$ and their centralizers. The methods are similar and we will need the following lemma.

(19.7) Let $G, \sigma$ be as above and $t \in C_{G}(\sigma) \cap Z(U)$. Write $Q=O_{2}\left(C_{G}(t)\right)$ and assume that $C_{Q / Z(Q)}(\sigma)=C_{Q}(\sigma) / Z(Q)$. Then $C_{G}(\sigma t)=C_{G}(\sigma) \cap C_{G}(t)$.

Proof. First we note that there is a root $r \in \Delta^{+}$such that $Z(U)=$ $Z\left(U_{r}\right)$ and $U_{r} \leq Q$. If $G \cong P S U(n, q), n$ odd, then $U_{r}$ is special of order $q^{3}$ and $\sigma$ inverts elements in $U_{r}-Z\left(U_{r}\right)$. Consequently the covering condition fails and this case is out. Thus $Z(U)=U_{r}$ and $\left|U_{r}\right|=q$. Then $C_{G}\left(U_{r}\right)=C_{G}(t)=Q\left(R \times H_{0}\right)$ where $H_{0} \leq H, Q=O_{2}\left(C_{G}(t)\right)$ and $R^{\sigma}=R$ is a Chevalley group satisfying $U=Q(U \cap R)$.

Since $(U \cap R)^{\sigma}=U \cap R$ the covering condition on $Q$ forces $C_{U}(\sigma)$ to cover $U / Z(Q) \cap C(\sigma)=U / U_{r} \cap C(\sigma)$. Let $x \in C_{G}(\sigma t)$ and write $x=\ell_{1} w \ell_{2}$ with $\ell_{1}, \ell_{2} \in B, w \in N$. Then $\ell_{1}^{\sigma t} t w^{\sigma} t \ell_{2}^{\sigma t} \in B w^{\sigma} B$. Since $C_{N}(\sigma)$ covers $C_{W}(\sigma)$ we may assume that $w \in C_{N}(\sigma) \leq C_{G}(\sigma)$. Now we use the Bruhat decomposition and write $B w B=B w U_{w}^{-}$, where $U_{w}^{-}=U \cap U^{w_{0} w}$ and where $w_{0}$ is the word of greatest length in $\left\{s_{1}, \cdots, s_{n}\right\}$. So we may assume $x=$ $h_{1} u_{1} w u_{2}$ for $u_{1} \in U, h_{1} \in H, u_{2} \in U_{w}^{-}$.

First assume that $t \in U_{w}^{-}$. We then apply the uniqueness of the Bruhat decomposition, obtaining $h_{1}^{\sigma t} u_{1}^{\sigma t} t=h_{1}^{\sigma t} u_{1}^{\sigma} t=h_{1} u_{1}$ and $t u_{2}^{\sigma t}=t u_{2}^{\sigma}=$ $u_{2}$. Read these equations modulo $U_{r} \unlhd B$. We conclude that $h_{1}^{\sigma}=h_{1}$ and using the covering condition we also have $u_{1}^{\sigma}=u_{1}, u_{2}^{\sigma}=u_{2}$. So we are done in this case.

Next assume that $t \in U_{w}=U \cap U^{w}$. Then $h_{1} u_{1} t w t u_{2}=h_{1} u_{1} t t^{w-1} w u_{2}$ and $t^{w-1} \in U$. Conjugating by $\sigma t$ we get the equations $h_{1}^{\sigma t}=h_{1}, u_{1}^{\sigma} t t^{w-1}=$ $u_{1}, u_{2}^{o}=u_{2}$. Reading modulo $U_{r}$ we get $h_{1}^{o}=h_{1}$, so we need only show that $u_{1}^{o}=u_{1}$.

If $t^{w-1} \in U_{r}$, then we argue as above to get $U_{1}^{o}=U_{1}$. So we may assume that $t^{w-1} \in U_{s} \neq U_{r}$. Then $s$ and $r$ have the same length and $\left|U_{r}\right|=\left|U_{s}\right|=q$. We can factor $\bar{U}=U / U_{r}=\prod \bar{U} r_{i}$, where $r_{i} \in \Delta^{+}-\{r\}$ and where respresentations of elements are unique. Consequently $t^{w-1} \in$ $U_{s}$ for $s$ a root with $\left(U_{s^{\prime}}\right)^{\sigma}=U_{s}$ and $\bar{u}_{1}$ having non-trivial projection to $\overline{U_{s^{\prime}}}$. Otherwise $\overline{u_{1}^{\sigma} t^{w-1}}$ has more non-trivial factors than $\bar{u}_{1}$. However the action of $\sigma$ implies that $U_{s^{\prime}}(c)^{\sigma}=U_{s}(c)$ for each $c \in F_{q}$. This situation is impossible, proving the lemma. 
(19.8) Let $G=P S L(n, q), E_{6}(q), P S U(n, q)$, or ${ }^{2} E_{6}(q)$, and let $\sigma$ be the graph automorphism as in (19.7). Let $r$ be in $\Delta^{+}$such that $Z\left(U_{r}\right)=Z(U)$ and let $t \in Z\left(U_{r}\right)^{\#}$.

i) If $G=P S L(n, q)$ or $P S U(n, q)$ for $n$ odd, then all involutions in Go are conjugate to $\sigma$.

ii) Otherwise each involution in $G \sigma$ is conjugate to either $\sigma$ or $\sigma$ t.

(19.9) Let $G, \sigma, t$ be as in (19.7).

i) If $G=P S L(n, q)$ or $P S U(n, q)$ for $n$ odd, then $C_{G}(\sigma) \cong \operatorname{PSO}^{+}(n, q)$.

ii) If $G=P S L(n, q)$ or $\operatorname{PSU}(n, q)$ for $n$ even, then $C_{G}(\sigma) \cong P \operatorname{PS}(n, q)$ and $C_{G}(\sigma t)$ is isomorphic to the centralizer of a transvection in $P S p(n, q)$.

iii) If $G=E_{6}(q)$ or ${ }^{2} E_{6}(q)$, then $C_{G}(\sigma) \cong F_{4}(q)$ and $C_{G}(\sigma t)$ is isomorphic to the centralizer of a central involution in $F_{4}(q)$.

In cases (ii) and (iii) Go contains precisely 2 conjugacy classes of involutions in Aut $(G)$.

We note that (19.9) follows from (19.7) and (19.8) once the covering condition appearing in (19.7) has been checked.

We let $r$ be as in (19.8) and $t \in Z\left(U_{r}\right)^{\#}$ (recall that $U_{r}=Z\left(U_{r}\right)$ unless $G=P S U(n, q)$ for $n$ odd). Then $P=N\left(Z\left(U_{r}\right)\right)$ is a parabolic subgroup of $G$ and $O^{2^{\prime}}(P)=C_{G}(t)=Q L$ where $Q=O_{2}(P)$ and $L \cong S L(n-2, q)$, $S L(6, q), S U(n-2, q)$, or $S U(6, q)$, accordingly as $G \cong P S L(n, q), E_{6}(q)$, $P S U(n, q)$, or ${ }^{2} E_{6}(q)$ (see [7], Sections 3, 4). Moreover we can choose $L$ such that $U \cap L \in \operatorname{Syl}_{2}(L)$ and $L$ is $\sigma$-invariant.

In each case $Q$ can be factored as a product of root subgroups, $Q=\prod_{i=1}^{k} U_{r_{i}}$, and $\bar{Q}=Q / Z\left(U_{r}\right)=\bar{U}_{r_{1}} \times \cdots \times \bar{U}_{r_{k}}$. If $\left|U_{r_{i}}\right|=q$ and $U_{r_{i}}^{\sigma}$ $=U_{r_{i}}$, then $\sigma$ centralizes $U_{r_{i}}$. On the other hand if $\left|U_{r_{i}}\right|=q^{2}$ and $U_{r_{i}}^{o}$ $=U_{r_{i}}$, then $U_{r_{i}}(c)^{\sigma}=U_{r_{i}}\left(c^{q}\right)$. Finally if $\left|U_{r_{i}}\right|=q^{3}$, then $r_{i}=r, C_{U_{r}}(\sigma)=$ $Z\left(U_{r}\right)$ and $\left|C_{\bar{U}_{r}}(\sigma)\right|=q$. If $G \neq P S L(n, q)$ for $n$ odd, then $U_{r_{i}}^{\sigma}=U_{r_{j}} \neq U_{r_{i}}$ implies that $\left[U_{r_{i}}, U_{r_{j}}\right]=1$. From this information one can verify the covering condition of (19.7) in each of the relevant cases.

What remains is the

Proof of (19.8) First we note that if $G=P S L(3, q)$ or $P S U(3, q)$ then (19.8)(i) is well-known. For $s \in \Delta$ let $Z_{s}=\Omega_{1}\left(U_{s}\right)\left(Z_{s}=U_{s}\right.$ unless $G=P S U(n, q), n$ odd) and set $X=\left\langle Z_{r}, Z_{-r}\right\rangle$.

Let $j$ be an involution in $G \sigma$. As $C_{G\langle\sigma\rangle}\left(Z_{r}\right)=Q L\langle\sigma\rangle$, we may assume that $j$ centralizes $Z_{r}, j \in Q L\langle\sigma\rangle$. We claim that for some $g \in G\left\langle Z_{r}^{g}, Z_{r}^{g j}\right\rangle$ $\sim X$. To see this it suffices by (12.1) to find $g \in G$ such that $Z_{r}^{g}, Z_{r}^{g j}$ do 
not generate a 2-group. This we do by induction on the rank of $G$. If $G=S L(3, q)$ or $S U(3, q)$ we use the fact that (19.8)(i) holds and check this directly. Otherwise consider $j \in Q L\langle\sigma\rangle$ and note that $\sigma$ induces a graph automorphism on $L$. Consequently we let $j$ act on $Q L / Q$ and apply induction.

By the above we may assume that $j$ normalizes $X$ and conjugating by an element of $X \cong S L(2, q)$ and using the fact that $O^{2}\left(C_{G}(X)\right)=L$ we may assume that $t j \in L\langle\sigma\rangle$. Inductively we have $j \sim t u \sigma$ where $u=1$ or $U_{s}(1)$, for $s$ the root of highest height in the room system $\Delta_{1} \subset \Delta$ of $L$. Moreover $u=1$ if $G=\operatorname{PSL}(n, q)$ or $\operatorname{PSU}(n, q)$ with $n$ odd.

Say $G=P S L(n, q)$ or $P S U(n, q)$ with $n$ odd. Then $j \sim t \sigma$. If $G=$ $P S U(n, q)$ then $\sigma$ acts on $U_{r}$ and inverts a homocyclic subgroup of order $q^{2}$ and exponent 4. It follows that $t \sigma \sim \sigma$. If $G=P S L(n, q)$, then $U_{r}=$ $\left[U_{s_{1}}, U_{s_{2}}\right]$ where $s_{1}=\alpha_{1}+\cdots+\alpha_{(n-1) / 2}, s_{2}=s_{1}^{\sigma}=\alpha_{(n+1) / 2}+\cdots+\alpha_{n}$. Then $\sigma$ acts on $U_{s_{1}} U_{s_{2}} U_{r}$ and again $\sigma \sim \sigma t$.

For the remaining cases we are done if $u=1$. Consequently we may assume $j \sim t U_{s}(1) \sigma$. We now perform simple computations to obtain the result. For $G=P S L(n, q), r=\alpha_{1}+\cdots+\alpha_{n-1}$ and $s=\alpha_{2}+$ $\cdots+\alpha_{n-2}$. Then $\sigma$ centralizes $U_{\alpha_{1}}(1) U_{\alpha_{n-1}}(1)=g$ and $\left(t U_{s}(1)\right)^{g}=U_{s+\alpha_{1}}(1)$ $U_{s+\alpha_{n-1}}(1) U_{s}(1)$. As $\sigma$ interchanges $U_{s+\alpha_{1}}(1)$ and $U_{s+\alpha_{n-1}}(1)$ we have $j \sim$ $\left(U_{r}(1) U_{s}(1) \sigma\right)^{g} \sim U_{s}(1) \sigma \sim t \sigma$ as desired.

Suppose $G=E_{6}(q)$. Then $s=\alpha_{1}+\alpha_{3}+\alpha_{4}+\alpha_{5}+\alpha_{6}$. Conjugating tuo by $s_{2} s_{4} s_{1} s_{6} s_{3} s_{5} s_{4} s_{3} s_{5} s_{1} s_{6} s_{2} \in C(\sigma)$ we have $j \sim U_{\alpha_{4}}(1) U_{\alpha_{3}+\alpha_{4}+\alpha_{5}}(1) \sigma$. Considering $\left\langle U_{ \pm \alpha_{3}}, U_{ \pm \alpha_{4}}, U_{ \pm \alpha_{5}}\right\rangle \cong S L(4, q)$ we use the above to get $j \sim U_{\alpha_{3}+\alpha_{4}+\alpha_{5}}(1) \sigma$ and hence $j \sim t \sigma$.

Finally, suppose that $G={ }^{2} E_{6}(q)$ or $\operatorname{PSU}(n, q)$ for $n$ even. Then there is an element $w \in W$ such that $\left\{r^{w}, s^{w}\right\}=\left\{\alpha_{2}, \alpha_{2}+2 \alpha_{3}\right\},\left\{\alpha_{n}, \alpha_{n}+2 \alpha_{n-1}\right\}$. Then $w \in C(\sigma)$ and we have $t u \sigma \sim U_{\alpha}(1) U_{\alpha+2 \beta}(1) \sigma$, where $\left\langle U_{\alpha}, U_{\beta}\right\rangle=$ $U_{\alpha} U_{\beta} U_{\alpha+\beta} U_{\alpha+2 \beta}$ is isomorphic to a Sylow 2-subgroup of $\operatorname{PSU}(4, q)$. Then $\left(U_{\alpha}(1) U_{\alpha+2 \beta}(1) \sigma\right)^{U \beta^{(1)}}=U_{\alpha}(1) U_{\alpha+\beta}(1) \sigma$ and conjugating by $U_{-\beta}(c)$, for $c \in F_{q}^{2}$ with $c+c^{q}=1$, we have $t u \sigma \sim U_{-\beta}(1) \sigma$. Finally the action of $\sigma$ on $U_{-\beta}$ described above together with (19.4) shows that $U_{-8}(1) \sigma \sim \sigma$. This completes the proof of (19.8).

\section{Section 20. Hypothesis II for Chevalley groups of characteristic 2.}

Let $A$ be a perfect central extension of a Chevalley group $\bar{A}=A / Z(A)$ defined over a field of characteristic 2 . We will use our information on 
involutions in Aut $(\bar{A})$ to prove

(20.1) Let $T$ be an elementary abelian 2-group acting on $A$ and $K \leq T A$ such that

i) $m_{2}(T)>1$.

ii) $T \in \operatorname{Syl}_{2}(K)$ and $K=O(K) T$.

iii) $T \cap C(A)=1$.

iv) $K$ is tightly embedded in $T A$.

Then $T \leq A C(A)$.

(20.1) is called Hypothesis 2 in [1] and this result will be essential in [3].

Assume (20.1) is false and $A$ is a counterexample. We will use the following notation: $K_{0}=O_{2^{\prime}}(K) \leq A$ and $T_{0}=T \cap A C(A)$. Since $A C(A) / C(A) \cong \bar{A}$ we will let bars denote projections of subsets of $A C(A)$ to $\bar{A}$, when no confusion arises from this abuse.

(20.2) $\bar{A}$ has $(B, N)-r a n k n>1$.

Proof. This can be easily checked from the known structure of Aut $(A)$.

(20.3) $\quad T_{0} \neq 1$

Proof. Suppose $T_{0}=1$. Then $m_{2}(T)>1$ implies that Out $(A)$ contains a klein group and the Dynkin diagram of $\bar{A}$ is simply laced. By Griess [13] either $\bar{A} \cong L_{3}(4)$ or $Z(A)$ has odd order. In the latter case $C_{A}(t)$ covers $C_{\bar{A}}(t)$ for each $t \in T^{\#}$. This also holds if $\bar{A}=L_{3}(4)$ since $C_{A}(t)$ covers $O^{2}\left(C_{\bar{A}}(t)\right)=C_{\bar{A}}(t)$ for each $t \in T^{\#}$. Also for $t \in T^{\#}\left[C_{\bar{A}}(t), T\right] \leq$ $C_{\bar{A}}(t) \cap K$, and so $T$ centralizes $C_{\bar{A}}(t) / O_{2^{\prime}, 2}\left(C_{\bar{A}}(t)\right)$ for each involution $t \in T^{\#}$. Now $T$ contains involutions $t_{1}, t_{2}$ such that $t_{1}$ is in the coset of a field automorphism of $\bar{A}$ and $t_{2}$ is in the coset of a graph-field automorphism of $\bar{A}$. However (19.1) and (19.5) show this to be impossible.

(20.4) $\quad N_{A}(K)$ is 2-constrained.

Proof. $K=O(K) T$. By (20.3) we may choose $t \in T_{0}^{\sharp}$. Then $t$ must centralize $E\left(N_{A}(K) / O\left(N_{A}(K)\right)\right)$ and so $O_{2^{\prime}, E}\left(N_{A}(K)\right)=X=O(X) C_{X}(t)$. As $C_{A}(t) \leq N_{A}(K), C_{X}(t) \unlhd C_{A}(t)$. But $O_{2^{\prime}, E}\left(C_{A}(t)\right)=O(C(A))$ and so $O_{2^{\prime}, E}(N(K))$ $=O(N(K))$ proving the lemma.

(20.5) $C_{A}(t)$ is 2-constrained for each $t \in T^{\#}$. 
Proof. $L(C(t)) \leq L(N(K))=1$ by (2.2) and (20.4).

Knowing (20.5) we can immediately use the information in Section 19 to eliminate many possibilities for $\bar{A}$. Let $j_{1} \in T-A C(A)$. We then have

(20.6) $T=\left\langle T_{0}, j_{1}\right\rangle$ and either $\bar{A} \cong P S O^{ \pm}(n, q)$ with $n \geq 8$ even and $j_{1}$ of type $b_{\ell}$ for some $\ell$, or letting $\sigma, t$ be as in (19.8) (and replacing $G$ by $\bar{A}$ ) we may assume that $\bar{A}=P S L(n, q) n$ even, $P S U(n, q) n$ even, $E_{6}(q)$, or ${ }^{2} E_{6}(q)$, and $j_{1}=$ ot .

Proof. Use (20.5) and the results in Section 19.

(20.7) $T \unlhd K$ and $T$ is tightly embedded.

Proof. We first claim that $C_{\bar{A}}(T)$ contains a klein group. Let $j \in T_{0}^{\#}$. Then $C_{\bar{A}}(j)$ is 2-constrained and $O_{2^{\prime}}\left(C_{\bar{A}}(j)\right)=1$ (by (2.4)). Also $\bar{T}_{0} \leq C_{\bar{A}}(j)$ so assume that $T_{0}=\langle j\rangle$. $\quad C_{A}(j)$ covers $O^{2}\left(C_{\bar{A}}(j)\right)$. So $\left[O^{2}\left(C_{\bar{A}}(j)\right), T\right] \leq$ $O^{2}\left(C_{\bar{A}}(j)\right) \cap T_{0} K$. Since $O_{2^{\prime}}\left(C_{\bar{A}}(j)\right)=1$ we must have this commutator contained in $T_{0}=\langle j\rangle$. It follows that $O^{2}\left(C_{\bar{A}}(j)\right) \leq C_{\bar{A}}(T)$. So if the claim is false $O^{2}\left(C_{\bar{A}}(j)\right)$ has 2-rank $\leq 1$ and is 2-constrained. The only possibility is $O^{2}\left(C_{\bar{A}}(j)\right)=1$ or $S L(2,3)$. The latter case contradicts the known structure of $C_{\bar{A}}(j)$, and $O^{2}\left(C_{\bar{A}}(j)\right)=1$ forces $\bar{A}=L_{3}(2)$ where we also obtain a contradiction.

Let $U_{0}$ be a klein group in $C_{\bar{A}}(T)$. Then $O(K)=\Gamma_{1, U_{0}}(O(K))$ by (2.5), so it suffices to show that $Q=C_{0(K)}(u)=1$ for each $u \in U_{0}^{\#}$. We have $Q=\Gamma_{1, T}(Q)$ by (2.5) so we need only show $P=C_{Q}(t)=1$ for each $x \in T^{\#}$. But as $C_{A T}(x)$ is 2-constrained (2.4) implies that $P \leq O\left(C_{A T}(u)\right)=1$. This proves (20.7).

At this point we may replace $K$ by $T$.

(20.8) If $Z(A)$ has odd order, then $C_{\bar{A}}(x) \leq N_{\bar{A}}(T)$ for each $x \in T^{\#}$. In any case $\left[O^{2}\left(C_{\bar{A}}(x)\right), T\right] \leq T$.

Proof. If $Z(A)$ has odd order, then $C_{\bar{A}}(x)=\overline{C_{A}(x)}$ and (20.7) gives the result. The second statement is similarly proved using $\left.\overline{O^{2}\left(C_{A}(x)\right.}\right)=$ $O^{2}\left(C_{\bar{A}}(x)\right)$.

(20.9) If $\bar{A} \not \operatorname{PSO}^{ \pm}(n, q)$, then $t^{a} \in \bar{T}_{0}$, where $t$ is as in (20.6), for some $\bar{a} \in \bar{A}$ and $C_{\bar{A}}(t)=C_{\bar{A}}\left(t^{a}\right)$.

Proof. We take $j_{1}=\sigma t$ as in (20.6) and consider $C_{\bar{A}}\left(j_{1}\right)$. From (19.8) 
we have $C_{\bar{A}}\left(j_{1}\right)$ isomorphic to the centralizer of a transvection in $P S p(n, q)$ or to the centralizer of an element of a root group in $F_{4}(q)$, depending on whether $\bar{A}$ is as in (19.8) ii) or (19.8) iii). In either case $O^{2}\left(C_{\bar{A}}\left(j_{1}\right)\right)=$ $C_{\bar{A}}\left(j_{1}\right)$, so (20.8) implies that $C_{\bar{A}}\left(j_{1}\right) \leq N_{\bar{A}}(T)$. Consequently $\bar{T}_{0} \unlhd C_{\bar{A}}\left(j_{1}\right)=Y$.

We consider the structure of $C_{\bar{A}}\left(j_{1}\right)$ (see [7], (3.2) and (4.5)). In either case $Z(Y)$ is a root group of $\bar{A}$. Given any normal subgroup $J \leq Y$ either $Z(Y) \leq J$ or $J \leq Z(Y)$. Consequently $\bar{T}_{0} \cap Z(Y) \neq 1$ and the result follows.

$$
\bar{A} \not P S L(n, q) n \text { even, } \operatorname{PSU}(n, q) n \text { even, } E_{6}(q) \text {, or }{ }^{2} E_{6}(q) \text {. }
$$

Proof. Let $j_{1}=\sigma t$ as in (20.6) and $t^{a} \in \bar{T}_{0}$ as in (20.9). Let $v \in T_{0}^{\#}$ such that $\bar{v}=t^{a}$. By (20.8) and (20.9) $O^{2}\left(C_{\bar{A}}(v)\right)=O^{2}\left(C_{\bar{A}}(t)\right) \leq N_{\bar{A}}(T)$. In particular $j_{1}$ centralizes $X=E\left(C_{\bar{A}}(v) / O_{2}\left(C_{\bar{A}}(v)\right)\right)$. However from the definitions of $\sigma$ and $t$ we see that $\sigma$ induces an involutory outer automorphism of $X$. This is a contradiction.

We are left with the use $\bar{A} \cong P S O^{ \pm}(n, q) n \geq q$ even and $j_{1}$ inducing $b_{\ell_{1}}$ for some $\ell_{1}$ (see (20.6)). Consequently we may assume $\bar{A}\left\langle j_{1}\right\rangle=\bar{A} T=$ $O^{ \pm}(n, q)$. Accordingly we let $M$ be the associated $n$-dimensional space over $F_{q}$ having a non-degenerate quadratic form preserved by $\bar{A}$. Let $j \in T_{0}^{\#}$ and $P_{j}$ the stabilizer in $\bar{A}$ of $[M, j]$. Then $P_{j}$ is a parabolic subgroup of $\bar{A}$ with $P_{j}^{j_{1}}=P_{j}$. Let $V=O^{2}\left(C_{\bar{A}}(j) / O_{2}\left(C_{\bar{A}}(j)\right)\right)$. Then $V$ is the central product of certain linear groups as described in Section 8 .

We first note that $V^{j_{1}}=V$ and also (20.8) implies that $\left[O^{2}(V), T\right] \leq$ $V \cap T=1$. Consequently $j_{1}$ centralizes $O^{2}(V)$. On the other hand $P_{j}$ is a maximal parabolic subgroup obtained by omitting the $\ell^{\text {th }}$ node of the Dykin diagram. Consequently $O^{2}\left(P_{j} / O_{2}\left(P_{j}\right)\right)$ is the central product of $S O^{ \pm}(n-2 \ell, q)$ and $S L(\ell, q)$. Suppose $\bar{j}$ is of type $a_{\ell}$. Then from (8.6) we have $V$ the central product of $S O^{ \pm}(n-2 \ell, q)$ and $S p(\ell, q)$. As $j_{1}$ centralizes $O^{2}(V), j_{1}$ centralizes the factor isomorphic to $S O^{ \pm}(n-2 \ell, q)$ in $O^{2^{\prime}}\left(P_{j} / O^{2}\left(P_{j}\right)\right)$, and since $j_{1}$ is a graph automorphism of $G$, we must have $n-2 \ell \leq 2$. We also note that since $C_{\bar{A}}(x)$ is 2-constrained for each $x \in T^{\#}$ we must have $j_{1}$ and $j_{1} j$ inducing outer automorphisms of $\bar{A}$ of type $b_{\ell_{1}}, b_{\ell_{2}}$ with $\ell_{1}>1$ and $\ell_{2}>1$.

Now $N_{\bar{A}}\left(\bar{T}_{0}\right) \leq P$ where $P$ is a proper parabolic subgroup of $\bar{A}$, the stabilizer of a singular subspace, $M_{0}$, of the natural module $M$. Using the known structure of $C_{\bar{A}}\left(j_{1}\right)$, and $C_{\bar{A}}\left(j_{1} j\right)$ given in (8.7) one verifies that 
$O^{2}\left(C_{\bar{A}}\left(j_{1}\right)\right)$ and $O^{2}\left(C_{\bar{A}}\left(j_{1} j\right)\right)$ stabilize unique isotropic subspaces of $M$ of dimensions $\ell_{1}-1, \ell_{2}-1$, respectively. Consequently $\ell_{1}-1=\ell_{2}-1=$ $\operatorname{dim}\left(M_{0}\right)$. Similarly if $\bar{j}$ has type $c_{\ell}$ then from (8.9) we see that $O^{2}\left(C_{\bar{A}}(\bar{j})\right)$ stabilizes precisely two isotropic subspaces of $M$ of dimensions 1 and $\ell-1$. As $\operatorname{dim}\left(M_{0}\right)=\ell_{1}-1$ is even, this is impossible. Consequently $\bar{j}$ has type $a_{\ell}$ and from earlier remarks $n-2 \ell \leq 2$.

Next we use (8.6) to check that $C_{\bar{A}}(\bar{j})$ stabilizes precisely one isotropic subspace of $M$ having dimension $\ell$. If $n-2 \ell=2$, then $O^{2}\left(C_{\bar{A}}(\bar{j})\right)$ will stabilize isotropic subspaces of dimensions $\ell$ and $\ell+1$. As $O^{2}\left(C_{\bar{A}}(\bar{j})\right) \leq P$ we have $\ell=\ell_{1}-1=\ell_{2}-1$ in all cases. Note that if $n=8$, then we must have $\ell_{1}=\ell_{2}=3$, so $\ell=2$. This contradicts $n-2 \ell \leq 2$. Consequently $n>8, Z(A)$ has odd order and $C_{\bar{A}}(j), C_{\bar{A}}\left(j_{1}\right), C_{\bar{A}}\left(j_{1} j\right)$ are all in $P$. Moreover $\ell \geq 4$ and since $2 \ell_{1} \leq n$ we have $n=2 \ell_{1}=2 \ell+2$.

Let $\mathscr{B}=\left\langle x_{1}, \cdots, x_{n}\right\rangle$ be an ordered basis for $M$ as in (8.2), so that $\bar{j}$ is in orthogonal Suzuki form relative to $\mathscr{B}$. Then $M_{0}=[M, \bar{j}]=\left\langle x_{1}\right.$, $\left.\cdots, x_{\ell}\right\rangle$ is invariant under $C_{\bar{A}}\left(j_{1}\right)$ and it follows from (8.3) that $M_{0}=$ $\left\langle v \in\left[M, \bar{j}_{1}\right]: Q(v)=0\right\rangle$. Then $j_{1}$ stabilizes $M_{0}^{\perp}=\left\langle x_{1}, \cdots, x_{\ell}, x_{\ell+1}, x_{\ell+2}\right\rangle=$ $\left\langle x_{1}, \cdots, x_{n-\ell}\right\rangle$. From (8.3) we see that there is another basis $\left\{x_{1}, \cdots, x_{\ell}\right.$, $x, y\}$ of $M_{0}^{\perp}$ such that $(x) j_{1}=1$ and $(y) j_{1}=x+y, Q(x)=1,(x, y)=1$, and $\langle x, y\rangle$ is non-degenerate. With respect to the basis $\mathscr{B}^{\prime}=\left\{x_{1}, \cdots, x_{\ell}, x\right.$, $\left.y, x_{n-\ell+1}, \cdots, x_{n}\right\} \bar{j}$ is still in Suzuki symplectic form and $j_{1}$ has matrix form

$$
\left(\begin{array}{llll}
I_{\ell} & & & \\
& 1 & & \\
& 1 & 1 & \\
B & \beta & & I_{\ell}
\end{array}\right)
$$

for some $\ell \times \ell$ matrix $B$ and column vector $\beta$. We have $C_{\bar{A}}(\bar{j}) \leq N_{\bar{A}}(T)$. Suppose $\beta \neq 0$. Let

$$
P=\left(\begin{array}{cccc}
1 & 0 & \cdots & 0 \\
0 & 0 & \cdots & 0
\end{array}\right) \text { and } Q=\left(\begin{array}{cc}
0 & 1 \\
0 & 0 \\
\vdots & \vdots \\
0 & 0
\end{array}\right)
$$

be $2 \times \ell$ and $\ell \times 2$ matrices and let $g \in C_{\bar{A}}(\bar{j})$ be the matrix

$$
\left(\begin{array}{lll}
I_{\ell} & & \\
P & I_{2} & \\
0 & Q & I_{\ell}
\end{array}\right) .
$$


Then $\bar{j}_{2}=\bar{j}_{1}^{j} \bar{j}_{1}$ satisfies $\operatorname{dim}\left[M, \bar{j}_{2}\right]=2$ and so $C_{\bar{A}}\left(\bar{j}_{2}\right) \not \subset P$. As $\bar{j}_{2} \in \bar{T}$, this is a contradiction.

\section{Section 21. Coverings of classical groups.}

Hypothesis 21.1. $A$ is a quasisimple group such that $Z(A)$ is an elementary abelian 2-group and $A / Z(A)=\bar{A}$ is a classical group $L_{n}(q)$, $U_{n}(q), S p_{n}(q)$, or $\Omega_{n}^{i}(q), n \geq 4, q$ even. Exclude the cases where $\bar{A}$ is $L_{4}(2) \cong A_{8}$ or $S p_{4}(2) \cong S_{6}$. If $\bar{A}$ is orthogonal take $n \geq 8$.

If $Z(A) \neq 1$ then results of Steinberg and Greiss (eg. [13]) show $\bar{A}$ to be $U_{4}(2), U_{6}(2), \Omega_{8}^{+}(2)$, or $S p_{6}(2)$.

Hypothesis 21.2. $T$ is a 4-group in $A$ with $T \cap Z(A)=1$ such that for each $t \in T^{\#}, T \leq O_{2}\left(C_{A}(t)\right)$ and either

i) $\left[T, t^{A} \cap C_{A}(t)\right]=1$, or

ii) $T \unlhd C_{A}(t)$.

In this section we prove

(21.3) Let $A$ satisfy hypothesis 21.1 with $Z(A) \neq 1$. Then there exists no 4-group $T$ in $A$ satisfying hypothesis 21.2 .

Assume 21.3 to be false and let $T$ be a counter example in $A$. Let $t \in T^{\sharp}$. We consider the four possibilities for $\bar{A}$ separately.

(21.4) $\bar{A} \not U_{4}(2)$.

Proof. Assume $\bar{A} \cong U_{4}(2)$. Then $A \cong S p(4,3)$. Then there is a unique class $t^{A}$ of involutions with $t \in A-Z(A)$. Further $\langle t\rangle Z(A)$ is the unique normal 4-group in $C_{A}(t)$.

(21.5) $\bar{A} \nRightarrow U_{6}(2)$.

Assume $\bar{A} \cong U_{6}(2)$. By Section $6, \bar{A}$ has 3 classes of involutions with representatives $j_{1}, j_{2}$, and $j_{3}$. Next

(21.6) $|Z(A)| \leq 4$ and $A$ admits a group of automorphisms transitive on $Z(A)^{\#}$ and fixing each class of involutions in $\bar{A}$.

So in particular there is a unique covering $B$ of $\bar{A}$ such that $Z(B)$ $=\langle\pi\rangle$ is of order 2 . The following facts appear in [16]. $B$ has 6 classes of involutions $\pi, d, d \pi, r, r \pi$, and $n$, where $\bar{d}=j_{1}, \bar{r}=j_{2}$, and $\bar{n}=j_{3}$. In particular each involution $\bar{x}$ in $\bar{A}$ lifts to an involution $x$ of $B$ and if $\bar{x}$ is fused to $j_{1}$ or $j_{2}$ then $C_{\bar{A}}(\bar{x})=\overline{C_{B}(x)}$ while $\left|C_{\bar{A}}(\bar{n}): \overline{C_{B}(n)}\right|=2$. Hence 
by 21.6 if follows that

(21.7). Let $Z(A)=\left\{z_{i}: 1 \leq i \leq m=|Z(A)|\right\}$. There are $2 m+1$ classes of involutions in $A-Z(A)$ with representatives $d z_{i}, r z_{i}$, and $n, 1 \leq i \leq m$, where $\bar{d}=j_{1}, \bar{r}=j_{2}$, and $\bar{n}=j_{3}$. If $x$ is fused to $d z_{i}$ or $r z_{i}$ then $C_{\bar{A}}(\bar{x})$ $=\overline{C_{A}(x)}$, while if $x \sim n$ then $\left|C_{\bar{A}}(\bar{x}): \overline{C_{A}(x)}\right|=m$.

It follows from 21.7 and 10.6 that we may take $t=n$ and $s \sim n$ for each $s \in T^{\#}$. Next, calculating using Section 6 we find $C_{\bar{A}}(\bar{n})=\bar{Y} \bar{X}$ where $\bar{Y}$ is elementary of order $2^{9}$ and $\bar{X} \cong U_{3}(2)$ is the extension of an elementary group $\bar{W}$ of order 9 by a quaternion group $\bar{Q}$. Further $\bar{Y}$ is generated by involutions $\bar{y}$ of rank 1 or 2 , so $C_{\bar{A}}(\bar{y})=\overline{C_{A}(y)}$ and hence $\Phi(Y)=1$. As $T \leq O_{2}\left(C_{A}(t)\right)=Y, Y$ centralizes $T$. As $\left|C_{\bar{A}}(\bar{n}): \overline{C_{A}(n)}\right|=$ $m \leq 4$, each involution $u$ with $\bar{u} \in \bar{Q}$ centralizes $t$. Thus if $v$ is an involution with $[\bar{v}, \bar{t}]=1$ then $[v, t]=1$. So by 10.6 we conclude $T \unlhd C_{A}(t)$. Then $W$ centralizes $T$. But $\bar{W}$ acts without fixed points on $\bar{Y} /\langle\bar{t}\rangle$, a contradiction. This completes the proof of 21.5.

(21.8) $\bar{A} \not \Omega_{8}^{+}(2)$.

Assume $\bar{A} \cong \Omega_{8}^{+}(2)$, Then by Section $8, \bar{A}$ has five classes of involutions with representatives $a_{2}, c_{2}, c_{4}, a_{4}$, and $a_{4}^{\prime}$. Next

(21.9) $|Z(A)|=m \leq 4$ and if $m=4$ then $A$ admits a group of automorphisms transitive on $Z(A)^{\#}$, fixing $a_{2}$ and $c_{4}$, and permuting $c_{2}, a_{4}$, and $a_{4}^{\prime}$ transitively.

So in particular there is a unique covering $B$ of $\bar{A}$ with $Z(B)=\langle\pi\rangle$ of order 2. From [11] and [15] we find there are 4 classes of involutions in $B-Z(B)$ with representatives $a, b, c$, and $c \pi$, where $\bar{a}=a_{2}, \vec{b}=$ $c_{4}$, and $\bar{c}=c_{2}$. $\quad a_{4}$ and $a_{4}^{\prime}$ lift to elements of order 4 . Therefore with 21.9 we conclude

(21.10) If $Z(A)=\langle\pi\rangle$ then there are 4 classes of involutions in $A-Z(A)$ with representatives $a, b, c$, and $c \pi$, where $\bar{a}=a_{2}, \bar{b}=c_{4}$, and $\bar{c}=c_{2}$. If $|Z(A)|=4$ then $a$ and $b$ are representatives for the classes in $A-Z(A)$, with $\bar{a}=a_{2}$ and $\bar{b}=c_{4}$.

If $|Z(A)|=2$, then $C_{\bar{A}}(\bar{c})=\overline{C_{A}(c)}$, so by 10.8 we conclude $t \not c$ or $c \pi$. Suppose $t=a$. Calculating using Section 8 we find $O^{2}\left(C_{\bar{A}}(\bar{a})\right)=\bar{X} \bar{Y}$ where $\bar{Y}=O_{2}\left(C_{\bar{A}}(\bar{a})\right)=\left\langle\bar{Y} \cap \bar{a}^{\bar{A}}\right\rangle$ is extraspecial of order $2^{9}$ and $\bar{X}$ is of 
order 27 and acts without fixed points on $\bar{Y} /\langle\bar{a}\rangle$. As $O^{2}\left(C_{\bar{A}}(\bar{a})\right)=O^{2}\left(\overline{C_{A}(a)}\right)$, we have a contradiction. Therefore

(21.11) $s \sim b$ for each $s \in T^{\#}$.

So we may take $t=b$. Let $W=O^{2}\left(C_{A}(t)\right)$ and $Q=O_{2}(W)$. Then calculating using Section 8 we find

(21.12) $\bar{Q}=\left\langle\bar{t}^{\bar{A}} \cap \bar{Q}\right\rangle$ with $Z(\bar{Q})=O_{2}\left(C_{\bar{A}}(\bar{t})\right) \cap C(\bar{Q})=\langle\bar{t}\rangle \times[Z(\bar{Q}), \bar{X}]$ where $X \in \operatorname{Syl}_{3}\left(C_{A}(t)\right)$. Moreover $\bar{t}^{\bar{A}} \cap Z(\bar{Q})=\bar{t}[Z(\bar{Q}), \bar{X}]$.

Now if $\left[t^{A} \cap C(t), T\right]=1$ then by $21.12, \bar{T} \leq Z(\bar{Q})$. On the other hand if $T \unlhd C_{A}(t)$ then $X$ centralizes $T$ and then $W=\left\langle X^{C(t)}\right\rangle \leq C(T)$. So again $\bar{T} \leq Z(\bar{Q})$. But this is impossible since by 21.11, $\bar{T}^{\#} \subseteq \bar{t}^{\bar{A}} \cap C(\bar{t})$, while by $21.12, \bar{t}^{\bar{A}} \cap Z(\bar{Q}) \subseteq \bar{t}[Z(\bar{Q}), \bar{X}]$. This completes the proof of 21.8 .

(21.13) $\bar{A} \not S p_{6}(2)$.

Assume $\bar{A} \cong S p_{6}(2)$. By Section $7, \bar{A}$ has 4 classes of involutions with representatives $b_{1}, b_{3}, a_{2}$, and $c_{2}$. $S p_{6}(2)$ has a multiplier of order 2, so $A$ is the covering group of $S p_{6}(2)$. Let $\langle\pi\rangle=Z(A)$. By [8], Theorem 3.12

(21.14) $A$ has 3 classes of involutions with representatives $\pi$, $a$, and $b$, where $\bar{a}=a_{2}$ and $\bar{b}=b_{3}$.

Next by lemmas 3.16 and 3.17 in [8]

(21.15) $\quad C_{A}(b)=\langle b\rangle \times B$ where $B$ is isomorphic to the centralizer in $M_{12}$ of a 2-central involution of $M_{12}$, and $\pi \in B$. Moreover $O_{2}(B)=\left\langle b^{A} \cap O_{2}(B)\right\rangle$.

In particular $\langle b, \pi\rangle$ is the unique normal 4-subgroup of $C_{A}(b)$ and the only 4-group centralized by $b^{A} \cap C(b)$. Therefore

(21.16) $s \sim a$ for each $s \in T^{\#}$.

Thus we may take $t=a$. Let $g, h \in A$ with

$$
\bar{g}=\left|\begin{array}{rrrrrr}
1 & & & & \\
1 & 1 & & & & \\
& & 1 & & & \\
& & 1 & & \\
& & & & & 1 \\
& & & & 1 & 1
\end{array}\right| \quad \bar{h}=\left|\begin{array}{llllll} 
& 1 & & & & \\
1 & & & & & \\
& & 1 & & & \\
& & 1 & & \\
& & & & 1 \\
& & & & 1
\end{array}\right| .
$$


Then $h \in a^{A}$ and $g$ is an element of order 3 inverted by $h$. Further $\overline{a h}$ is of type $b_{3}$ and hence by $21.14 a h$ is an involution. Thus $h \in C(a)$. So $g=[g, h]$ must centralize $T$. However an easy calculation shows $\bar{g}$ centralizes an involution $\bar{u}$ in $O_{2}(C(\bar{a}))$ exactly when $Q(\bar{u})$ is

$$
\left|\begin{array}{ll}
1 & 0 \\
0 & 1
\end{array}\right| \quad\left|\begin{array}{ll}
1 & 1 \\
1 & 0
\end{array}\right| \quad\left|\begin{array}{ll}
0 & 1 \\
1 & 1
\end{array}\right|
$$

In particular $a$ and $a \pi$ are the only members of $a^{G} \cap O_{2}(C(a))$ centralized by $g$.

This completes the proof of 21.3 .

\section{Section 22. Tightly embedded subgroups of the classical groups.}

Hypothesis 22.1. $G=T A=C_{G}(A) A$ where $A=A(q)$ satisfies hypothesis 21.1, $C_{G}(A)$ is an elementary abelian 2-group, $C_{T}(A)=1=\Phi(T)$, $m(T)>1$, and $T$ is tightly embedded in $G$. Further either

(1) $|T|=4$, or

(2) $T^{g} \cap N(T) \leq C(T)$ for each $g \in G$.

Let $P$ be a complement to $Z(A)$ in the preimage in $A$ of the projection of $T$ on $\bar{A}=A / Z(A)$, containing $T \cap A$. Let $t \in T^{\#}$ with $\ell$ the rank of t. Set $\{p\}=P \cap t C(A)$.

In this section we prove

(22.2) Assume hypothesis 22.1. Then $Z(A)=1, P^{\sharp}$ is fused in $A$ and either

(I) $P \leq J=O_{2}\left(C_{A}(p)\right) \cap C\left(p^{A} \cap C(p)\right),|T| \leq q$, and one of the following holds :

(1) $J=\alpha(p)$ and $\operatorname{Aut}_{A}(J)$ is cyclic of order $q-1$ and regular on $J^{\#}$.

(2) $A=S p_{n}(q), p=b_{\ell}, \ell>1, J=\alpha(a) \alpha(b)$ where $a$ and $b$ are of type $a_{\ell-1}$ and $b_{1}$, respectively, and $\operatorname{Aut}_{A}(J) \cong Z_{q-1} \times Z_{q-1}$ is regular on $J-(\alpha(a) \cup \alpha(b))$.

(3) $A=S p_{n}(q), p=c_{\ell}, J=\alpha(a) \alpha(b)$ where $a$ and $b$ are of type $a_{\ell}$ and $b_{1}$, respectively, and $\operatorname{Aut}_{A}(J)$ is as in (2).

(4) $A=\Omega_{n}(q), p=c_{\ell}, J=\beta(p)$ and $\operatorname{Aut}_{A}(J)$ is cyclic of order $q-1$ and regular on $J^{\#}$.

or,

(II) $T=P$ has order 4 , and either 
(5) $A=S L_{n}(2), t=j_{2}$, and $T \leq \Phi(S), S \in \operatorname{Syl}_{2}\left(C_{A}(t)\right)$.

(6) $A=S p_{n}(2)$, and $t=c_{2}$.

Proof. If 22.1 .2 holds then $\left[P, p^{A} \cap C_{A}(p)\right]=\left[T, p^{A} \cap C_{A}(p)\right]=1$. Suppose 22.1.2 does not hold. Then 22.1.1 holds so $T$ and $P$ are 4groups. Moreover $T=\langle t, s\rangle$ and $s^{a}=s t$, some $a \in C_{A}(t)$. Let $\{u\}=$ $s C(A) \cap P$. Then $s=c u, c \in C(A)$, and $c u^{a}=s^{a}=s t=c u t$, so $u^{a}=u t$. In particular $P \unlhd C_{A}(t)$. Therefore hypothesis 21.2 is satisfied in $A$ by any 4-group in $P$. So by $21.3, Z(A)=1$.

Now suppose $P \leq J$. Then as hypothesis 21.2 is satisfied, the results in Section 10 and Section 11 imply one of (1)-(4) occurs. Also $\operatorname{Aut}_{A}(J)$ is transitive on $P^{A} \cap J$ so has the form indicated.

Next suppose $P \not J$. Then 22.1.2 is not satisfied, so as shown above, $T$ and $P=\langle u, t\rangle$ are of order 4 with $u^{C(t)}=\{u, u t\}$. So by Section 10, $P$ is a known subgroup of $A$. Suppose $A=U_{n}(q)$. By 10.6, $A=U_{n}(2)$, $\ell=2$, and $u \in Z_{\ell}$ with

$$
Q(u)=\left[\begin{array}{ll}
1 & 0 \\
0 & 0
\end{array}\right] \text { or }\left[\begin{array}{ll}
0 & 0 \\
0 & 1
\end{array}\right]
$$

But then $u$ is a transvection, so $P$ is not normal in $C_{A}(u)$. The same argument eliminates the possibility that $A=S p_{n}(2)$ and $t=a_{2}$ or $t=c_{2}$ with

$$
Q(u)=\left[\begin{array}{ll}
1 & 1 \\
0 & 0
\end{array}\right] \text { or }\left[\begin{array}{ll}
0 & 1 \\
0 & 1
\end{array}\right]
$$

Therefore Section 10 implies $P^{\#}=T^{\#}$ is fused and either $A=S L_{n}(2)$ and $t=j_{2}$ or $A=S p(n, 2)$ and $t=c_{2}$. By 4.6, if $A=S L_{n}(2)$ then $T \leq \Phi(S)$, $S \in \operatorname{Syl}_{2}\left(C_{A}(t)\right)$.

In Case II we have shown $P^{\#}$ to be fused. Moreover in cases (1) and (4), $P^{\#}$ is fused. Consider cases (2) and (3) and assume $P^{\#}$ is not fused. Then $P^{\#}$ contains an element $v$ of type $b_{1}$ or $a_{k}$. But then by what we have shown, $P$ does not centralize $v^{A} \cap C(v)$, a contradiction. So in general, $P^{\#}$ is fused.

\section{BIBLIOGRAPHY}

[1] Aschbacher, M., Tightly embedded subgroups of finite groups, (to appear).

[2] Aschbacher, M., On finite groups of component type, Ill. J. Math., 19 (1975), $87-115$.

[ 3 ] Aschbacher, M. and Seitz, G., On groups with a standard component of known type, (to appear). 
[ 4 ] Borel, A. and Tits, J., Éléments unipotents et sousgroupes paraboliques de groupes réductifs, I, Inventiones Math., 12 (1971), 95-104.

[ 5 ] Bourbaki, N., Groupes et algebres de Lie, Chap. 4, 5, 6 Fascicule XXXIV, Elements de Math., Paris, Hermann 1968.

[ 6 ] Carter, R., Simple groups of Lie type, Wiley, New York, 1972.

[ 7 ] Curtis, C. Kantor, W., and Seitz, G., The two transitive permutation representations of the finite Chevalley groups, Trans. A. M. S., 218 (1976), 1-59.

[ 8 ] Fendel, D., A characterization of Conway's group, 3, J. Alg., 24 (1973), 159-196.

[9] Feit, W., The current situation in the theory of finite simple groups, Actes. Congress Intern. Math., 1 (1974), 55-93.

[10] Fong, P., and Seitz, G., Groups with a $(B, N)$-pair of rank 2, I, II, Inventiones Math., 21, 24 (1973), (1974), 1-57, 191-239.

[11] Frame, J., The characters of the Weyl group of $E_{8}$, Computational Problems in Abstract Algebra, 111-130, Pergamon Press, Oxford, (1970) (Ed. J. Leech) .

[12] Gorenstein, D., Finite Groups, Harper and Row, New York, 1968.

[13] Griess, R., Schur multipliers of the finite simple groups of Lie type (to appear

[13] Griess, R., Schur multipliers of the finite simple groups of Lie type, Trans. A. M. S., 183 (1973), 355-421.

[14] Guterman, A characterization of the groups $F_{4}\left(2^{\mathrm{n}}\right)$, J. Alg., 20 (1972), 1-23.

[15] Hamill, C., A collineation group of order $2^{13} \cdot 3^{5} \cdot 5^{2} \cdot 7$, Proc. London Math. Soc., 3 (1953), 54-79.

[16] Hunt, D., A characterization of the finite simple groups M (22), J. Alg., 21 (1972), 103-112.

[17] Lang, S., Algebraic groups over finite field, Amer. J. Math., 18 (1956), 555-563.

[18] Parrot, D., A characterization of the Ree groups ${ }^{2} F_{4}$ (q), J. Alg., 27 (1973), 341357.

[19] Seitz, G., Flag-transitive subgroups of Chevalley groups, Annals of Math., 97 (1973), 27-56.

[20] Seitz, G., and Wright, C., On complements of F-residuals in finite solvable groups, Archiv. der Math., 21 (1970), 139-150.

[21] Steinberg, R., Automorphisms of finite linear groups, Canadian J. Math., 12 (1960), 606-615.

[22] Suzuki, M., Characterizations of linear groups, Bull, A. M. S., 75 (1969), 10431091.

[23] Thomas, G., A characterization of the group $\mathrm{G}_{2}\left(2^{\mathrm{n}}\right)$, J. Alg., 13 (1969), 87-118.

[24] Thomas, G., A characterization of the Steinberg group $D_{4}{ }^{2}\left(q^{3}\right), q=2^{n}$, J. Alg., 14 (1970), 373-385.

[25] Wall, G., On the conjugacy classes in the unitary, symplectic, and orthogonal groups, J. Australian Math. Soc., 3 (1963), 1-62. 